INTER NATIONAL MONETARY FUND
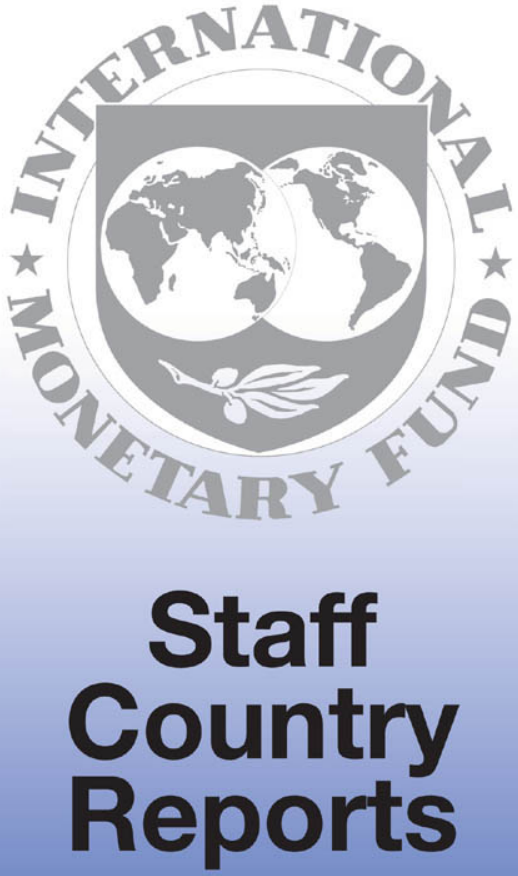


\section{Vietnam: Statistical Appendix and Background Notes}

This Statistical Appendix and Background Notes report on Vietnam was prepared by a staff team of the International Monetary Fund as background documentation for the periodic consultation with this member country. As such, the views expressed in this document are those of the staff team and do not necessarily reflect the views of the Government of Vietnam or the Executive Board of the IMF.

$$
\begin{aligned}
& \text { Copies of this report are available to the public from } \\
& \text { International Monetary Fund - Publication Services } \\
& 700 \text { 19th Street, N.W. - Washington, D.C. } 20431 \\
& \text { Telephone: (202) 623-7430 * Telefax: (202) 623-7201 } \\
& \text { Telex (RCA): } 248331 \mathrm{IMF} \text { UR } \\
& \text { E-mail: publications@imf.org } \\
& \text { Internet: http://www.imf.org } \\
& \text { Price: } \$ 15.00 \text { a copy }
\end{aligned}
$$

\section{International Monetary Fund Washington, D.C.}




\section{INTERNATIONAL MONETARY FUND}

\section{VIETNAM \\ Statistical Appendix and Background Notes}

Prepared by Peter Winglee and David Cowen (both APD), Saade Chami (PDR), and Jong-Won Yoon (FAD)

Approved by Asia and Pacific Department

July 7,2000

Contents

Page

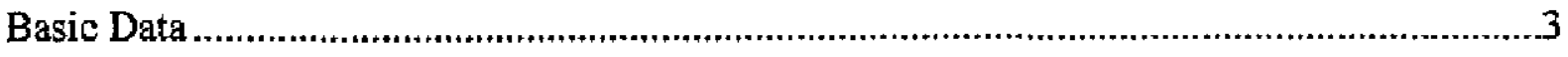

Tables

1. Gross Domestic Product by Expenditure Categories at Current Prices, 1993-99 ,........

2. Gross Domestic Product by Sector and Ownership at Current Prices, 1993-99 ...........5

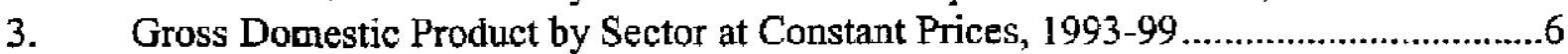

4. Consumer Price Inflation, 1995-2000 ....................................................................

5. Gross Value of Agricultural Production at Constant Prices, 1995-99 .........................8

6. Production of Food Staples, 1995-99 .................................................................9

7. Industrial Crop Production and Livestock, 1995-99 …..........................................10

8. Gross Value of Industrial Production at Constant Prices, 1995-99 ............................11

9. Industrial Production by Sector of Ownership at Constant Prices, 1995-99 ..............12

10. Population and Employment, 1995-99 ...............................................................13

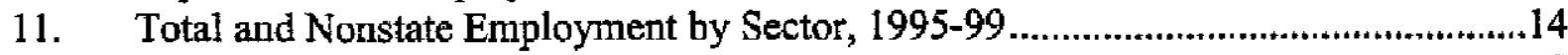

12. Employment in the State Sector, 1995-99 …...................................................15

13. Summary of General Government Budgetary Operations, 1995-2000 .....................16

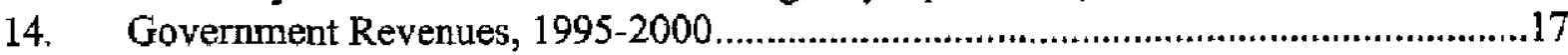

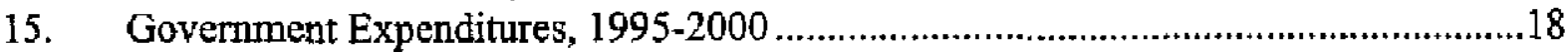

16. Treasury Bills and Bond Issues, 1996-2000 .............................................................19

17. Monetary Survey, 1995-2000 ............................................................................20

18. Balance Sheet of the State Bank of Vietnam, 1995-2000 .....................................21

19. Consolidated Balance Sheet of Deposit Money Banks, 1995-2000 ..........................22

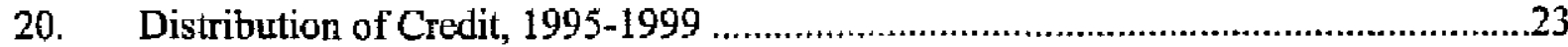

21. Overdue Loans of Deposit Money Banks, 1995-2000 ..........................................24

22. Nominal and Real Interest Rates, 1995-2000 .........................................................25

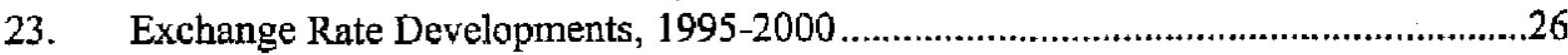

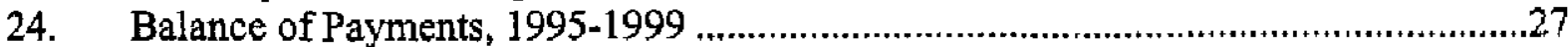

25. Merchandise Exports by Commodity, 1995-99 …..................................................28

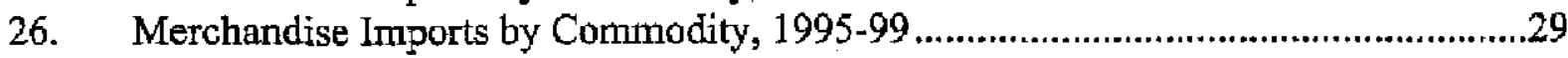

27. Direction of Trade, 1995-99 ................................................................................ 
28. Commitments of Foreign Direct Investment, 1995-99 ….......................................31

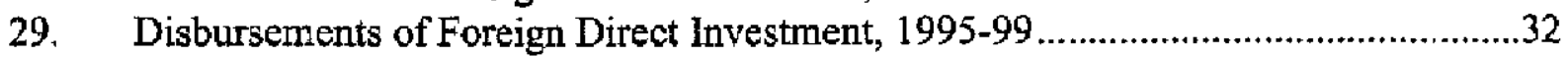

30. Foreign Direct Investment by Country of Origin, 1995-99 .......................................33

31. External Debt and Debt Service Obligations, 1995-99.........................................34

Background Notes

I. $\quad$ Recent Developments in the External Trade System................................................35

II. Recent Developments in the Foreign Exchange System .........................................40

III. Recent Export Performance ...................................................................................43

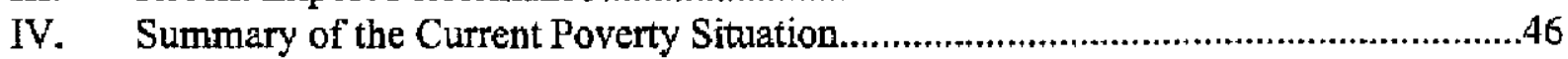

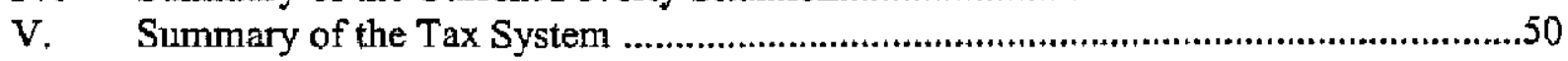


Vietnam: Basic Data

Nominal GDP (1999): US\$28.5 billion

Population (1999): $\quad 76.6$ million

GDP per capita (1999): USS3T2

Fund Quots:

SDR 329.1 million

\begin{tabular}{|c|c|c|c|c|c|}
\hline & 1995 & 1996 & 1997 & $\begin{array}{r}1998 \\
\text { Est. } \\
\end{array}$ & $\begin{array}{r}1999 \\
\text { Est. }\end{array}$ \\
\hline Real GDP (antiual percentinge change) & 9.5 & 9.3 & 8.2 & 3.5 & 4.2 \\
\hline Industrial output 1/ & 14.5 & 14.2 & 13.8 & 12.5 & 10.4 \\
\hline Savine-investment ludance (in percent of GDP) & -13.3 & -10.5 & -6.9 & -4.6 & 3.9 \\
\hline Gross nationsl saving & 11.9 & 159 & 19.8 & 17.1 & 24.4 \\
\hline Gross aspital formotion & 25.4 & 26.3 & 26.7 & 21.6 & 20.5 \\
\hline \multicolumn{6}{|l|}{ In fletion (antual percentage ohange) } \\
\hline Period average & 16.9 & 5.6 & 3.1 & 7,9 & 4.1 \\
\hline End of period & 129 & 4.4 & 3.6 & 9.2 & -0.2 \\
\hline GDP defiator & 17.0 & 8.7 & 6.6 & 11.1 & $5 . T$ \\
\hline \multicolumn{6}{|l|}{ Gowemment badjet (in percent of GDP) $2^{\prime}$} \\
\hline Total revenuc & 22.6 & 22.4 & 20.0 & 19.6 & 18.2 \\
\hline Grants & 0.7 & 0.6 & 0.8 & 0.6 & 0.5 \\
\hline Total expenditure (exeluding onlending) $3 /$ & 23.8 & 23.1 & 22.6 & 20.7 & 19.6 \\
\hline Of which: current expenditure & 18.5 & 17.4 & 16.3 & 15,0 & 13.2 \\
\hline Overall fiscal balance (including grasts, exciuding onlending) 3 f & -0.5 & -0.2 & -1.7 & -0.5 & -0.9 \\
\hline \multicolumn{6}{|l|}{ Maney and erwilh (andusl percentage change, end of period) } \\
\hline Broad money & 22.6 & 22.7 & 26.1 & 25.6 & 39.3 \\
\hline Credit to the economy & 26.9 & 20.1 & 22.6 & 16.4 & 19.2 \\
\hline \multicolumn{6}{|l|}{ Interest rabes (in peroent, end of period) } \\
\hline Three-month deposits (households) & 18.2 & 9.1 & 8.1 & 9.7 & 4.0 \\
\hline Short-term lending (less than one year) & 28.3 & 15.9 & 12.7 & 14.7 & 11.7 \\
\hline \multicolumn{6}{|l|}{ Cument account (including offioinl transfors) } \\
\hline (in millions of L.S. dollats) & $-2,648$ & $-2,431$ & $-1,664$ & $-1,067$ & 1,252 \\
\hline (in percent of GDP) & -12.8 & -9.9 & -6.2 & -3.9 & 4.4 \\
\hline Exports of good (anumal perceratage change, U.S. dollat terms) & 28.2 & 41.2 & 24.6 & 2.4 & 23.2 \\
\hline Imports of goods (aruual percentage change U.S. dollar terms) & 41.1 & 25.5 & $=0.2$ & -1.1 & 1.1 \\
\hline \multicolumn{6}{|l|}{ Foneign exclukne reserves (in millions of U.S. dollars, end of period) } \\
\hline Gross official reserves, including gold & 1,323 & 1,673 & 1,857 & 1,765 & 2,711 \\
\hline (in weeks of next year's imparts of pood and nonfactor services) & 5.2 & 6.4 & 7.2 & 6.7 & 9.1 \\
\hline Net internationsl rescrves, including gold & 946 & 1,136 & 1,350 & 1,259 & 2,197 \\
\hline Extentl debt (in percent of GDP) 4 I & 85.1 & 78.1 & 77.7 & 76.8 & 74.0 \\
\hline Convertible currency 5 / & 35,0 & 36.6 & 38.6 & 38.1 & 37.1 \\
\hline Nonconvertible currency & S1.1 & 41.5 & 39.1 & 3B,8 & 36.9 \\
\hline \multicolumn{6}{|l|}{ Delbe-service ratto (percent of exports of goods and nonfuotor services) } \\
\hline Debt due & 12.1 & 9.8 & 11.1 & 13.2 & 10.7 \\
\hline Debt peid & 6.7 & 5.6 & 7.8 & 8.1 & --- \\
\hline \multicolumn{6}{|l|}{ Exchange rate (dong per U.S. dollar) } \\
\hline Period average & 11,038 & 11,033 & 11,706 & 13,297 & 13,944 \\
\hline End of period & 11,015 & 11,150 & 12,202 & 13,896 & 14,028 \\
\hline \multicolumn{6}{|l|}{ Resl effective excharige sate (annual percentage change) } \\
\hline Period average & 6.5 & 6.6 & 3.3 & 8.2 & -6.2 \\
\hline End of period & 8.9 & 3.7 & 12.6 & -9.3 & -3.2 \\
\hline \multicolumn{6}{|l|}{ Momorandum itexns: } \\
\hline GDP (in trillions of dong at oument market prices) & 228.9 & 272.0 & 313.6 & 360.6 & 397.3 \\
\hline Per capita GDP (in U.S. dollars) & 287 & 336 & 360 & 359 & 372 \\
\hline
\end{tabular}

Sources: Dala provided by the authorities; and staff extimates and projections.

$1 /$ Annusel percentage change in gross valus of inchustrial output.

2/ Cash basis.

3/ Excludes capital costs of stato-owned enterprise mol banking sector reforms

4/ London Club rescheduling wo conetuded in early 1998.

S/ Includes the ban component of foreign direct investment and other private acctor borrowing, and short-term debt. 
Table 1. Vietnam: Gross Domestic Product by Expenditure Categories at Current Prices, 1993-99 1/

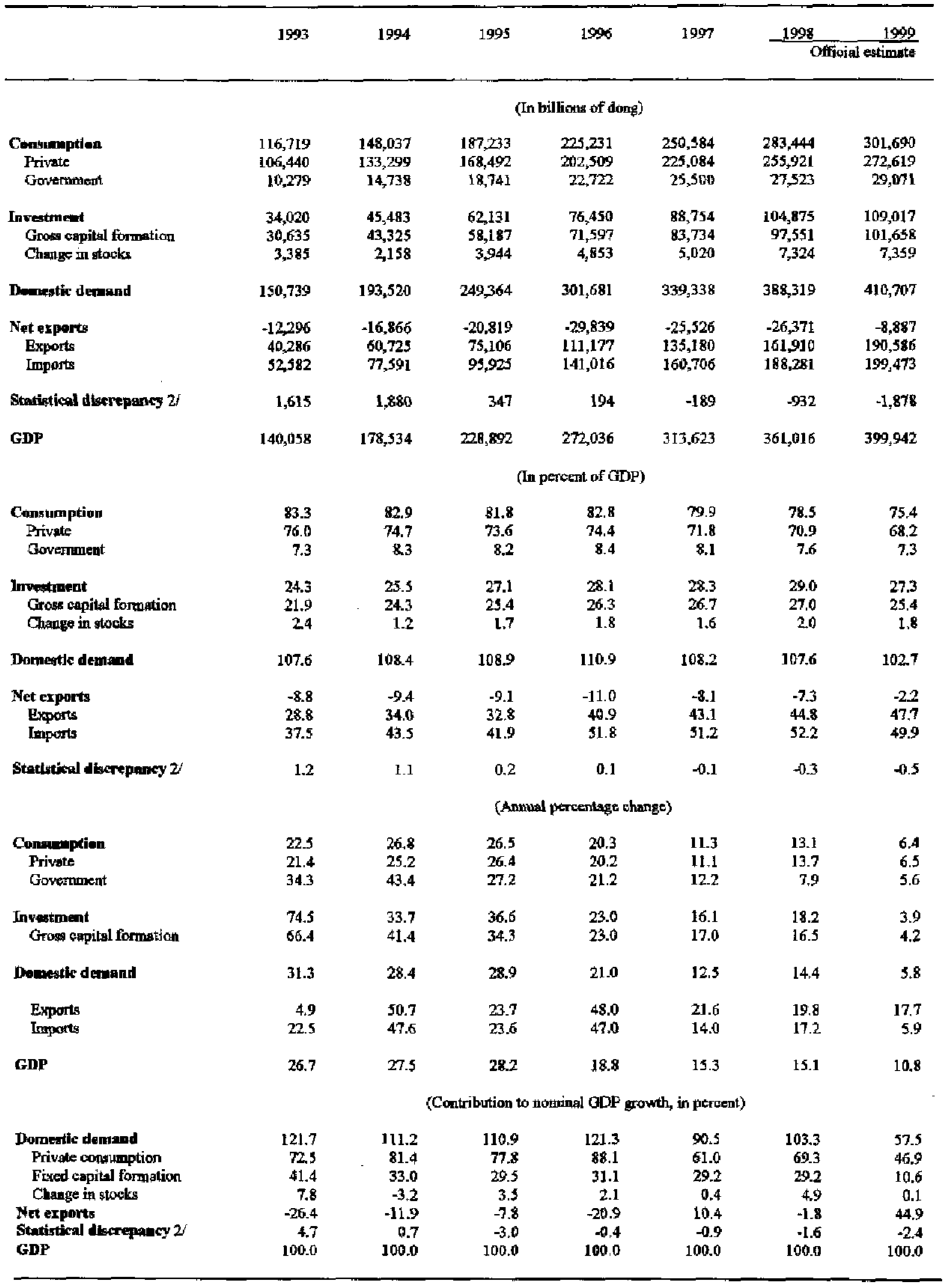

Source: General Statistical Office.

U/ Dath are latest of ticial revisions of historical GDP extimates.

2/ Difference betwein production and expenditure-based estimates of GDP, the fomer of which gre considered to be more aecurate. 
Table 2. Vietnam: Gross Donestic Product by Sector and Ownership at Current Prices, 1993-99 1/

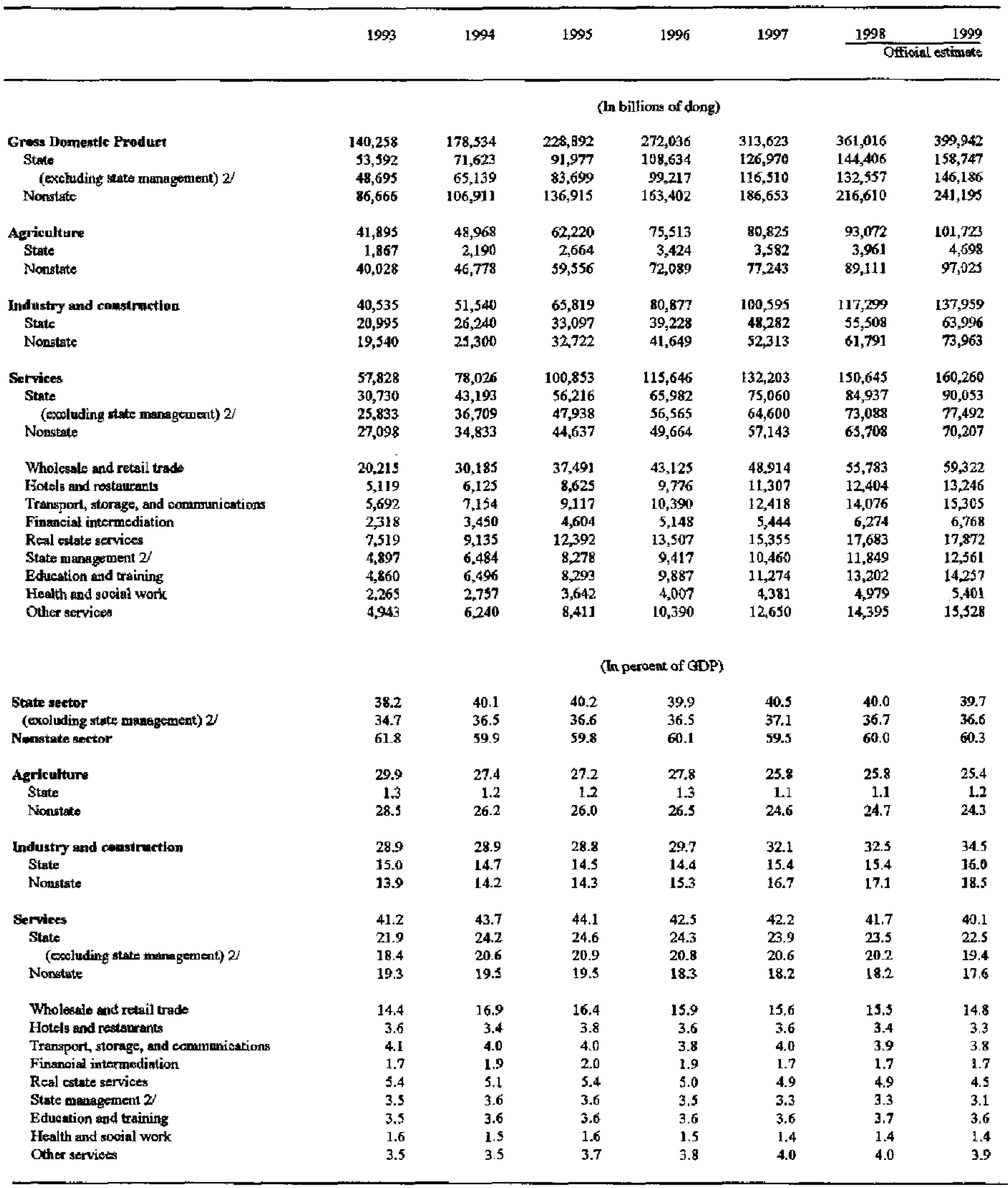

Source: General Statistival Office.

1/ Data are latest official revisions of historical GDP estimates.

2/ State management includes public adminustration, deferke, and compulsory social gecurity. 
Table 3. Vietran; Grou Domeatic Freduct by Sector gt Constant Prices, 1993-93 1/

\begin{tabular}{|c|c|c|c|c|c|c|c|c|c|}
\hline & \multirow[t]{2}{*}{1993} & \multirow[t]{2}{*}{2594} & \multirow[t]{2}{*}{1995} & \multirow[t]{2}{*}{1996} & \multirow[t]{2}{*}{$199 \mathrm{~T}$} & \multicolumn{2}{|c|}{1998} & \multicolumn{2}{|c|}{1999} \\
\hline & & & & & & $\begin{array}{l}\text { Official } \\
\text { entimtate }\end{array}$ & $\frac{\text { Staff }}{\text { estmate }}$ & $\begin{array}{l}\text { Offreial } \\
\text { estimate }\end{array}$ & $\begin{array}{c}\text { Stafi } \\
\text { estimate }\end{array}$ \\
\hline & \multicolumn{9}{|c|}{ (In piltions of dong at 1994 enonstant pricess] } \\
\hline Agriculture, forestry, and fichery & 47,373 & 48,968 & 51,319 & 53,577 & 53,895 & 57,866 & 37,544 & 60,892 & 60,554 \\
\hline Aqricallare & 40,428 & 41,839 & 43,658 & 43,652 & 47,925 & 49,639 & $\ldots$ & 52,370 & $\ldots$ \\
\hline Fot & 2,217 & 2,265 & 2,399 & 2,448 & 2,450 & 2,459 & $\cdots$ & 2,536 & $\cdots$ \\
\hline Fishery & 4,728 & 4,864 & 5,262 & 5.477 & 5,530 & 5,768 & $\cdots$ & 5,586 & $\ldots$ \\
\hline Industry & 45,454 & $\$ 1,5 \$ 0$ & $\$ 8.550$ & 67,016 & 75.473 & $\$ 1.764$ & 81,420 & 88,047 & 87,676 \\
\hline Mining and quarrying & 7,535 & 9,114 & 10,345 & 11,753 & 13,304 & 15,173 & $\ldots$ & 17,450 & ... \\
\hline Mamufacturing & 24,353 & 26,624 & 30,231 & 34,339 & 38,743 & 42,694 & $\ldots$ & 45,858 & $\ldots$ \\
\hline Eloctricity, gas, find water suppiy & 2,616 & 2,856 & 3,384 & 3,986 & 4,572 & 5,136 & $\ldots$ & 5,498 & $\ldots$ \\
\hline Constructioan & 10,950 & 12,946 & 14,590 & 16,938 & 18,855 & 28.761 & $\ldots$ & 19,211 & $\cdots$ \\
\hline services & 71,198 & 78,026 & 85,698 & 93,240 & $\$ 9,895$ & 104,966 & 100,393 & 107,390 & 101,297 \\
\hline Wholessle and retail trode & 27,807 & 30.185 & 33,505 & 36,866 & 39,422 & 41.170 & .. & 41,953 & $\ldots$ \\
\hline Hotols and restaurants & 5.719 & 6,125 & 6,741 & 7,428 & 7,949 & 8,307 & $\ldots$ & 8,457 & $\ldots$ \\
\hline Transport, storags, ind cormmtrnicatious & B.708 & 7,154 & 7,851 & 8,429 & 9,178 & 9,536 & $\ldots$ & 9,976 &.. \\
\hline Finsictal internediation & 2,800 & 3.450 & 3,940 & 4,388 & 4,578 & 4,843 & $\ldots$ & 5,006 & $\ldots$ \\
\hline Retl estate services & 8,390 & 9,135 & 9,738 & 30,337 & 11,071 & 11,682 & $\ldots$ & $1], 644$ & $\ldots$ \\
\hline Stute manggersent $2 /$ & 5,839 & 6,484 & 7,065 & 7,558 & 7,860 & 8,174 & $\ldots$ & 8,304 & $\ldots$ \\
\hline Edhacation thd training & 5,708 & 6,496 & 6,568 & 7,526 & 8,062 & 8,614 & $\ldots$ & 8,916 & $=$ \\
\hline Health and suovial work & 2,593 & 2,757 & 3.009 & 3,230 & 3,348 & 3,566 & $\ldots$ & 3,707 & $\ldots$ \\
\hline Other strvices & 5,614 & 6,240 & 6,792 & 7,487 & 8,427 & $\$, 074$ & $\cdots$ & $\$, 367$ &.- \\
\hline \multirow[t]{2}{*}{ Gross Domestic Produet } & 164,025 & 178.534 & 195,567 & 273,833 & 231,263 & 244,596 & 239,337 & 256,269 & 249,527 \\
\hline & \multicolumn{9}{|c|}{ (Real GDF, amulat percentage change) } \\
\hline Adricalbure, forestog, and flathery & 3.3 & 3.4 & 4.8 & 4.4 & 4.3 & 3.5 & 2.9 & 5.2 & 5.2 \\
\hline Agrieultare & 3.9 & 3.5 & 4.3 & 4.6 & 5.0 & 3.6 & $\ldots$ & 5.5 & $\ldots$ \\
\hline Foreary & -4.6 & 2.2 & 5.9 & 20 & 0.1 & 0.4 & $\ldots$ & 3.1 & $\ldots$ \\
\hline Finbery & 20 & 2.9 & 8.2 & 4.1 & 1.0 & $\mathbf{4 . 3}$ & $\cdots$ & 3.8 & 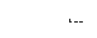 \\
\hline Inturtry & 12.6 & 13.4 & 13.6 & 14.5 & 12.6 & B.3 & 79 & 7.7 & 7.7 \\
\hline Mining and quarrying & $\ldots$ & 21.0 & 23.5 & 13.6 & 33.2 & 14.1 & .. & 15.0 & $\ldots$ \\
\hline Manufacturing & -21.6 & 9.3 & 13.5 & 13.6 & 72.8 & 10.2 & $\ldots$ & t.5 & $\ldots$ \\
\hline Electricily, gas, and water supply & $\ldots$ & 9.2 & 18.5 & 17.8 & 14.7 & 12.3 & $\ldots$ & 7.0 & $\ldots$ \\
\hline Construction & 17.3 & 182 & 32.7 & 16.1 & 11.3 & -05 & c.- & 2.4 & $\cdots$ \\
\hline Servicese & 8.6 & 9.6 & 9.8 & 8.8 & 7.1 & 5.1 & 0.5 & 2.3 & 0.9 \\
\hline Wholegale and retail trade & 6.1 & 8.6 & 11.3 & 9.7 & 6.9 & 4.4 & $\ldots$ & 1.9 & $\ldots$ \\
\hline Hotels and restaurants & -67.9 & $7+1$ & 10.1 & 102 & 7.0 & 4.5 & $\ldots$ & 1.8 & $\ldots$ \\
\hline Traysport, storase, and commumistions & 6.6 & 66 & 9.3 & 7.4 & 8.9 & 3.9 & $\ldots$ & 4.6 & $\ldots$ \\
\hline Brancial intennediation & 16.7 & 22.3 & 14.2 & 11.4 & 4,3 & 5.8 & $\ldots$ & 3.4 & $\ldots$ \\
\hline Reel estatio Earyloes & $\ldots$ & 8.9 & 6.6 & 6.1 & 7.1 & 5.5 & $\ldots$ & -0.3 & $\ldots$ \\
\hline Stale moningement 2 & $-\$ 4.3$ & 11.0 & 8.9 & 7.0 & 4.0 & $\$ 0$ & 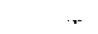 & 1.6 & $\ldots$ \\
\hline Educetion and training & $\ldots$ & 13.8 & 7.3 & 8.0 & 3.1 & 6.8 & $\ldots$ & 3.5 & $\ldots$ \\
\hline Health and roojal work & $\ldots$ & 6.3 & 9.1 & 3.0 & 4.0 & 6.5 & $\ldots$ & 4.0 & $\ldots$ \\
\hline Other servioes & $\ldots$ & $1 \mathrm{I} .2$ & 6.8 & 10.2 & 12.6 & 7.7 & $\cdots$ & 3.2 & $\cdots$ \\
\hline \multirow[t]{2}{*}{ Gron Domeatic Prod act } & 8.1 & 8.8 & 9.5 & 9.3 & 8.2 & 5.8 & 3.5 & 4.8 & 4.2 \\
\hline & \multicolumn{9}{|c|}{ (GDP dcflator, annual percentage oluange) } \\
\hline Agricalture, forestry, and rakery & 4.6 & 15.2 & 21.2 & 16.3 & 2.6 & 11.2 & $\cdots$ & 3.9 & $\ldots$ \\
\hline Agricutarts & 2.0 & 15.7 & 20.7 & 10.8 & $\mathbf{2 . 8}$ & 11.6 & $\ldots$ & 9.7 & $\ldots$ \\
\hline Forestry & 17.8 & 7.4 & 18.5 & 61.9 & 24 & 9.8 & $\ldots$ & 49 & $\ldots$ \\
\hline Fishery & 24.9 & $14 . *$ & 26.6 & 40.9 & 27 & 9.8 & $\ldots$ & 5.1 & $\ldots$ \\
\hline Inimitry & 15.2 & 11.7 & 12.4 & 7.4 & 10.4 & 7.6 & $\ldots$ & 9.2 & $\ldots$ \\
\hline Mining and quanrying & $\ldots$ & $\ldots$ & 6.4 & 22.2 & 14.3 & 7.3 & $\ldots$ & 22.9 & $\ldots$ \\
\hline Manufienthing & $\$ 4,9$ & -20.1 & 13.5 & 3.9 & 11.0 & 8.7 & $\ldots$ & 5.7 & $\ldots$ \\
\hline Elentritily, ges, and water supply & $\ldots$ & $\ldots$ & 38.9 & 78.1 & 14.7 & 7.0 & $\cdots$ & 5.3 & $m$ \\
\hline Contutruction & 38.2 & 7,4 & 8.2 & -3.1 & 3.8 & 2.3 & $\ldots$ & 1.5 & $\ldots$ \\
\hline Servilet: & 19.1 & 14.7 & 17.7 & $\$ .4$ & 6.7 & 8.4 & $\ldots$ & 4.0 & 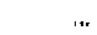 \\
\hline Wholesede and retal trate & 8.3 & 20.7 & 11.6 & 4.8 & 6.1 & 9.2 & $\ldots$ & 4.4 & ... \\
\hline Hotals and restaurante & 328.1 & -58.2 & 27.9 & 2.9 & 8.1 & 50 & $\ldots$ & 4.9 & $\ldots$ \\
\hline Tranepost, stortges and onmminioations & 21.5 & 7.2 & 16.1 & 6.1 & 9.8 & 9.1 & $\ldots$ & 3.9 & $\ldots$ \\
\hline Financial intermediation & 26,9 & 21.2 & 169 & 0.4 & 1.4 & 89 & $\ldots$ & 4.4 & $\ldots$ \\
\hline Real eatites services & $\cdots$ & $\ldots$ & $\ldots$ & 2.7 & 6.1 & 9.1 & $\ldots$ & 1.4 & $\ldots$ \\
\hline State managemont $2 f$ & 224.1 & -53.1 & 17.2 & 6.3 & 6.8 & 89 & $\ldots$ & 4.3 & $\ldots$ \\
\hline Education and training & $\cdots$ & $\ldots$ & $\therefore$ & 10.4 & 6.4 & 9.6 & $\cdots$ & 4.3 & $\ldots$ \\
\hline Hesthe and socrol work & $\cdots$ & $\cdots$ & $\cdots$ & 2.8 & 5.2 & 6.7 & $\ldots$ & 4.3 & $\ldots$ \\
\hline Other servigess & $\cdots$ & $\cdots$ & $\cdots$ & 12.] & 8.2 & 5.7 & $\cdots$ & 4,5 & $\ldots$ \\
\hline Grues Danestic Prodect & 13.4 & 14.0 & 17.0 & 8.7 & 6.6 & 8.8 & $\ldots$ & 5.7 & $\ldots$ \\
\hline
\end{tabular}

Sources: General Statistical Office; and staff estimates

1/ Data ave hatest offivial revisions of historical GDF' estinciales.

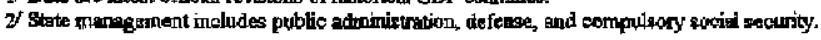


Table 4. Vletunaw: Cousumer Price InDation, 1995-2000 1/

(Annual anerage and 124xhonth pereentage changes, unless otherwise indicaled)

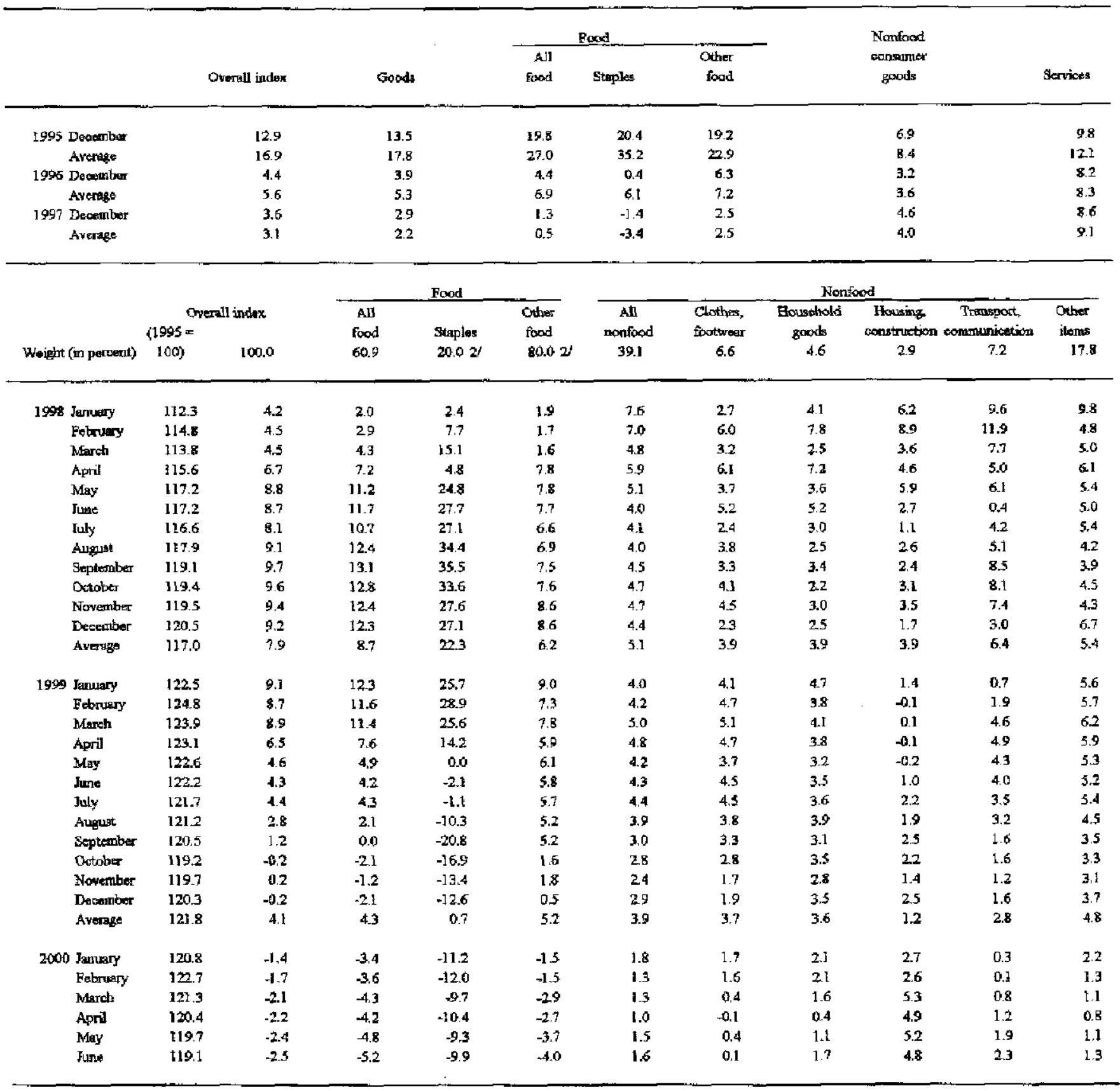

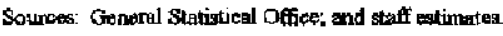

1/ Since lanuary 1998, the CPI index has been ealenlated under a new methodology and with different wights for the components. I/ Staff extingate. 
Table 5. Vietnam: Gross Value of Agricultural Production at Constant Prices, 1995-99

\begin{tabular}{|c|c|c|c|c|c|}
\hline & 1995 & 1996 & 1997 & 1998 & $\begin{array}{c}1999 \\
\text { Estimate }\end{array}$ \\
\hline & \multicolumn{5}{|c|}{ (In billions of dong, at constant 1994 prices) } \\
\hline Total & 79,812 & 83,967 & 89,958 & 93,502 & 100,283 \\
\hline Food crops & 54,034 & 56,814 & 59,942 & 62,257 & 65,969 \\
\hline Paddy and other cereals & 42,110 & 44,654 & 46,593 & 49,060 & 52,738 \\
\hline Vegetables and beans & 4,984 & 5,088 & 5,441 & 5,682 & 5,947 \\
\hline Fruits & 5,578 & 5,688 & 6,132 & 6,091 & 6,193 \\
\hline Dther & 1,362 & 1,384 & 1,775 & 1,424 & 1,091 \\
\hline Industrial crops & 12,149 & 12,806 & 14,551 & 15,042 & 16,977 \\
\hline \multirow[t]{2}{*}{ Animal husbandry } & 13,629 & 14,347 & 15,465 & 16,204 & 17,337 \\
\hline & \multicolumn{5}{|c|}{ (In percent total production) } \\
\hline Food crops & 67.7 & 67.7 & 66.6 & 66.6 & 65.8 \\
\hline Paddy and other cereals & 52.8 & 53.2 & 51.8 & 52.5 & 52.6 \\
\hline Vegetables and beans & 6.2 & 6.1 & 6.0 & 6.1 & 5.9 \\
\hline Fruits & 7.0 & 6.8 & 6.8 & 6.5 & 6.2 \\
\hline Other & 1.7 & 1.6 & 2.0 & 1.5 & 1.1 \\
\hline Industrial crops & 15.2 & 15.3 & 16.2 & 16.1 & 16.9 \\
\hline \multirow[t]{2}{*}{ Animal husbandry } & 17.1 & 17.1 & 17.2 & 17.3 & 17.3 \\
\hline & \multicolumn{5}{|c|}{ (Annual percentage change) } \\
\hline Total & 6.6 & 5.2 & 7,1 & 3.9 & 7.3 \\
\hline Food crops & 5.0 & 5,1 & 5.5 & 3.9 & 6.0 \\
\hline Paddy and other cereals & 4.7 & 6.0 & 4.3 & 5.3 & 7.5 \\
\hline Vegetables and beans & 10.0 & 2.1 & 6.9 & 4.4 & 4.7 \\
\hline Fruits & 2.7 & 2.0 & 7.8 & -0.7 & 1.7 \\
\hline Other & 6.1 & 1.6 & 28.3 & -19.8 & -23.4 \\
\hline Industrial crops & 17.6 & 5.4 & 13.6 & 3.4 & 12.9 \\
\hline Animal husbandry & 4.5 & 5.3 & 7.8 & 4.8 & 7.0 \\
\hline
\end{tabular}

Source: General Statistical Office. 
Table 6. Vietnarn: Productlon of Fowd Staples, 1995-99

\begin{tabular}{|c|c|c|c|c|c|}
\hline & 1995 & 1996 & 1997 & 1998 & $\begin{array}{c}1999 \\
\text { Estimnate }\end{array}$ \\
\hline & \multicolumn{5}{|c|}{ (In thousiands of tons) } \\
\hline Total produetion & 27,571 & 29,218 & 30.618 & 31,854 & 34,254 \\
\hline \multicolumn{6}{|l|}{ By prodinet } \\
\hline Rice padily & 24,964 & 26,397 & 27,524 & 29,146 & 31,394 \\
\hline Sping crop & 10,737 & 12,210 & $13, \mathbf{3 1 0}$ & 13,560 & 14,104 \\
\hline Autumit crop & 6,501 & 6,879 & 6,638 & 7,523 & 8,757 \\
\hline Whinter trop & 7,726 & 7.300 & 7,576 & 8,063 & 8,533 \\
\hline Other stoples (thet squiratent units) & 2,601 & 2,821 & 3,094 & 2708 & 2,860 \\
\hline Other staples (gross output) & 5,334 & 5,301 & 5,745 & 4,911 & 5,304 \\
\hline Maize & 1,177 & $1,53 ?$ & 1.651 & 1,612 & 1,752 \\
\hline Sweot potato & 1,686 & $1,69 ?$ & 1,691 & 1,526 & 1,745 \\
\hline Caseava & 2,212 & 2,057 & 2,403 & 1,773 & 1,807 \\
\hline Potatots & 260 & $\ldots$ & $\ldots$ & $\ldots$ & $\ldots$ \\
\hline \multicolumn{6}{|l|}{ By regian } \\
\hline North & 10,576 & 10,927 & 12,015 & 12,093 & 13,170 \\
\hline \multirow[t]{2}{*}{ South } & 16,995 & {$[8,29]$} & 18,603 & 19,761 & 21,084 \\
\hline & \multicolumn{5}{|c|}{ (Armunal pexcentage cherige) } \\
\hline Total production. & 52 & 60 & 48 & 40 & 7.5 \\
\hline Ripe paddy & 6.1 & 57 & 43 & 5.9 & 7.7 \\
\hline Spring crop & 22 & 137 & 90 & 1.9 & 4.0 \\
\hline Autumm crop & 15.5 & 5.8 & -35 & 13.3 & 16.4 \\
\hline Winter crop & 4.5 & -54 & $3:$ & 6.4 & 5.8 \\
\hline Other staples (rise equivalent units) & -2.4 & 82 & 97 & -12.5 & 5.6 \\
\hline Maize & 2.9 & 30.5 & $\because 4$ & -24 & 8.7 \\
\hline Sweat potato & -11.5 & 0.7 & -04 & -9.8 & 14,4 \\
\hline Cussara & -6.2 & -6.5 & 16.2 & -26.2 & 1.9 \\
\hline \multirow[t]{2}{*}{ Potatoes } & 0.2 & $\ldots$ & & $\cdots$ & $\ldots$ \\
\hline & \multicolumn{5}{|c|}{ (In thousendt of hearmes) } \\
\hline Toral area cultivated & 7.973 & 8,217 & 8,330 & 8,587 & 8,868 \\
\hline \multicolumn{6}{|l|}{ By product } \\
\hline Rice padity & 6,766 & 7,004 & 7,100 & 7,363 & 7,648 \\
\hline Spting crop & 2,421 & 2,541 & 2,683 & 2783 & 2,890 \\
\hline Autumin crop & 1,742 & 1,984 & 1,885 & 2,141 & 2,335 \\
\hline Wivter crop & 2,602 & 2,479 & 2,532 & 2,439 & 2,423 \\
\hline Other taples & 1,207 & 1,213 & 1,230 & 1,224 & 1.220 \\
\hline Maize & 557 & 615 & 663 & 650 & 687 \\
\hline Swet potsto & 305 & 303 & 267 & 254 & 265 \\
\hline Cassatra & 278 & 276 & 254 & 236 & 267 \\
\hline Other & 68 & 20 & 46 & 84 & 1 \\
\hline \multicolumn{6}{|l|}{ By reglon } \\
\hline North & 3,333 & $3,3,04$ & 3,365 & $3,3,46$ & 3,379 \\
\hline South & 4,640 & 4.913 & 4,965 & 5,241 & 5.489 \\
\hline Ylald & \multicolumn{5}{|c|}{ (In mrtric tans per heatare) } \\
\hline \multicolumn{6}{|l|}{ By prodect } \\
\hline Rice paddy & 3.7 & 9.8 & 3.9 & 4.0 & 4.1 \\
\hline Spring crop & 4.4 & 4.8 & 5.0 & 4.9 & 4.9 \\
\hline Autumin crop & 3.7 & 3.5 & 3.5 & 3.5 & 3.8 \\
\hline Winter ctop & 3.0 & 29 & 3.0 & 3.3 & 3.5 \\
\hline Maize & 2.1 & 2.5 & 2.5 & 2.5 & 2.6 \\
\hline Sweet potato & 5.5 & 5.6 & 6.3 & 6.0 & 6.6 \\
\hline Cancava & 80 & 7.5 & 9.5 & 7.5 & 6.8 \\
\hline \multicolumn{6}{|l|}{ By region } \\
\hline Worth & 3.2 & 3.3 & 3.6 & 3.6 & 3.9 \\
\hline South & 3.7 & 3,7 & 3.7 & 3.8 & 3.8 \\
\hline \multicolumn{6}{|l|}{ Per eapita production } \\
\hline Total staples (in metric tons per capits) & 0.382 & 0.398 & 0.411 & 0.422 & 0.449 \\
\hline Rice & 0.346 & 0.360 & 0.370 & 0.386 & $0.41\}$ \\
\hline Other staples & 0.036 & 0.038 & 0.042 & 0.036 & 0.037 \\
\hline (is percent of total siaples produption) & 9.5 & 9.7 & 10.3 & 8.5 & 8.3 \\
\hline Total staplos $(1994-300)$ & 103.5 & 108.0 & 111.5 & 114.3 & 121.7 \\
\hline Rios & 104.4 & 108.7 & 111.5 & 116.5 & 124.2 \\
\hline Other staples & 96.0 & 102.3 & 110.6 & 95.4 & 99.7 \\
\hline Total slaples (amusal pertantrge change) & 3.5 & 4.3 & 3.2 & 25 & 6.5 \\
\hline Rine & 4.4 & A.1 & 27 & 4.4 & 6.6 \\
\hline Other stoplos & -4.0 & 6.5 & 8.0 & -33.7 & 4.6 \\
\hline
\end{tabular}

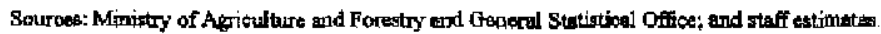


Table 7. Vietnam: Industrial Crop Production and Livetuck, 1995-99

\begin{tabular}{|c|c|c|c|c|c|}
\hline & 1995 & 1996 & 19,97 & 1998 & $\begin{array}{r}1999 \\
\text { Estimate }\end{array}$ \\
\hline & \multicolumn{5}{|c|}{ (In thousands of metric tons) } \\
\hline \multicolumn{6}{|l|}{ Production of nnnulal crops } \\
\hline Cotton & 12.8 & $\$ 1.2$ & 14.1 & 22.0 & 21.4 \\
\hline Jute & 14.8 & 15.0 & 223 & 14.6 & 9.1 \\
\hline Rush & 75.6 & 55.0 & 80.9 & 69.9 & 58.8 \\
\hline Sugareane & 10,711 & 1,372 & 11,921 & 13,844 & 17.840 \\
\hline Peacuts & 334 & 358 & 351 & 386 & 319 \\
\hline Soybeans & 126 & $1] 4$ & 113 & 147 & 145 \\
\hline Tobacto & 27.7 & 23.5 & 27.2 & 33.3 & 35.3 \\
\hline \multicolumn{6}{|l|}{ Preduction of perenula] crops } \\
\hline Tea & 40.2 & 46.8 & 52.2 & 56.6 & 64.7 \\
\hline Coffee & 218 & 320 & 421 & 400 & 487 \\
\hline Rubber & 125 & 145 & 187 & 194 & 215 \\
\hline Caconut & 1,165 & 1,318 & 1,318 & 1,106 & 1,134 \\
\hline \multirow[t]{2}{*}{ Pepper } & 9.3 & 10.5 & 13.0 & 15.9 & 17.8 \\
\hline & \multicolumn{5}{|c|}{ (Anatal persentage change) } \\
\hline Catton & 47.1 & -12.5 & 25.9 & 560 & -2.7 \\
\hline Jute & 15.6 & 1.4 & 4.3 & .34 .5 & -97.7 \\
\hline Ruath & 9.4 & -27.2 & 4.3 & -13.6 & -15.9 \\
\hline Sugarcane & 41.9 & 6.7 & 4.3 & 16.1 & 28.9 \\
\hline Peanuts & 13.6 & 7.0 & -0.2 & 9.9 & -17.4 \\
\hline Soybeans & 0.8 & -9.3 & -09 & 29.8 & -1.4 \\
\hline Tobaceo & 27.6 & -15.2 & 12.5 & 22.4 & 60 \\
\hline Tea & -4.3 & 16.4 & 10.6 & 8.4 & 14.3 \\
\hline Coffee & 21.1 & 46.8 & 31.6 & .27 & 18.9 \\
\hline \multirow[t]{2}{*}{ Rubber } & -3.2 & 143 & 30.8 & 14.4 & 14.4 \\
\hline & \multicolumn{5}{|c|}{ (In thousands of hectares) } \\
\hline Area cultivated of annual crops & 692 & 666 & 681 & 755 & $79 n$ \\
\hline Cotton & 18 & 15 & 15 & 24 & $\mathbf{n}$ \\
\hline Jute & 8 & 8 & 12 & 7 & 4 \\
\hline Rush & 10 & 9 & 11 & 10 & 9 \\
\hline Sugaroune & 225 & 237 & 237 & 283 & 351 \\
\hline Peasulss & 260 & 263 & 254 & 269 & 248 \\
\hline Soybeans & 121 & 110 & 106 & 129 & 129 \\
\hline Tobacoso & 28 & 24 & 25 & 32 & 33 \\
\hline Area cultivaled of perennial crops & 704 & 811 & 935 & 993 & 1,044 \\
\hline Teta & 67 & 75 & 79 & $\pi$ & 85 \\
\hline Cotfies & 186 & 254 & 340 & 371 & 397 \\
\hline Rubber & 278 & 303 & 348 & 382 & 394 \\
\hline Coconut & 173 & 180 & 170 & 163 & 168 \\
\hline \multirow[t]{2}{*}{ Pepper } & 7.0 & 7.5 & 9.8 & 12.8 & 15.0 \\
\hline & \multicolumn{5}{|c|}{ (In output per hectare, in metric tons) } \\
\hline Cotton & D. 7 & 0.7 & 0.9 & 0.9 & 1.0 \\
\hline Jute & 2.0 & 1.9 & 1.9 & 2.2 & 2.2 \\
\hline Rush & 7.3 & 6.0 & 7.3 & 7.1 & 6.3 \\
\hline Sugarcane & 47.6 & 48.0 & 46.4 & 48.9 & 50.9 \\
\hline Peanut & 1.3 & 1.4 & 1.4 & 1.4 & 1.3 \\
\hline Soybeans & 1.0 & 1.0 & 1.1 & 1.1 & 1.1 \\
\hline Tobacto & 1.0 & 1.0 & 1.0 & 1.0 & 1.1 \\
\hline Tes & 0.6 & 0.6 & 0.7 & 0.7 & 0.8 \\
\hline Coffet & 1.2 & 1.3 & 1.2 & 1.1 & 1.2 \\
\hline \multirow[t]{2}{*}{ Rubber } & 0.4 & 0.5 & o.s & 0.5 & 0.5 \\
\hline & \multicolumn{5}{|c|}{ (In millions of heads) } \\
\hline \multicolumn{6}{|l|}{ Livestock } \\
\hline Buffalo & 3.0 & 3.0 & 29 & 3.0 & 3.0 \\
\hline Cattle & 3.6 & 3.8 & 3.9 & 4.0 & 4.1 \\
\hline Pigrs (over two months old) & 16.3 & 16.9 & 17.6 & 18.1 & 18.9 \\
\hline Poultry & 142.1 & 151.4 & 160.6 & 166.4 & 179.3 \\
\hline
\end{tabular}

Sources: Ministry of Azriculture and Forestry atd General Statistical Office. 
Table 8. Vletnam: Gross Value of Industrial Production at Constant Prices, 1995-1999 if

\begin{tabular}{|c|c|c|c|c|c|}
\hline & 1995 & 1996 & 1997 & 1998 & $\begin{array}{c}1999 \\
\text { Estimate }\end{array}$ \\
\hline & \multicolumn{5}{|c|}{ (In trillions of dong, at constant 1994 prices) } \\
\hline Mining & 13.9 & 16.0 & 18.3 & 21.1 & 24.3 \\
\hline Coxi & 1.7 & 1.9 & 2.2 & 2.1 & 1.7 \\
\hline Oil and gas & 10.8 & 125 & 14.2 & 16.9 & 20.1 \\
\hline Metal ores & 0,2 & 0.3 & 0.2 & 0.2 & 0.2 \\
\hline Stone and other mining & 1.2 & 1.3 & 1.7 & 1.9 & 2.1 \\
\hline Manufacturing & 83.2 & 94.8 & 107.6 & 120.7 & 132.6 \\
\hline Food and beverages & 27.0 & 30.9 & 34.0 & 36.5 & 39,4 \\
\hline Cigarenes and tobacco & 4.0 & 4.2 & $4+4$ & 4.9 & 5.0 \\
\hline Textiles and garments & 9.1 & 9.8 & 11.6 & 13.0 & 14.3 \\
\hline Textile & 6.2 & 6.4 & 7.3 & 8.4 & 9.3 \\
\hline Garments & 2.9 & 3,4 & 4.3 & 4.6 & 5.0 \\
\hline Leather tanning and processing & 3.6 & 4.5 & 6.6 & 7.1 & 7.9 \\
\hline Wood and paper products & 5.2 & 5.5 & 3.8 & 6.2 & 6.3 \\
\hline Wood products & 3.3 & 32 & 3.2 & 3.0 & 28 \\
\hline Paper & 1.9 & 2.3 & 2.6 & 3.2 & 3.5 \\
\hline Printing, copying, publishing & 1.5 & 1.5 & 1.6 & 1.9 & 2.0 \\
\hline Chemical and petrolewn products & 5.4 & 6.5 & 7.3 & 8.2 & 9.1 \\
\hline Coke and petroleun & 0.3 & 0.2 & 0.1 & 0.1 & 0.1 \\
\hline Chemicals & 5.1 & 6.3 & 7.2 & 8.1 & 9.0 \\
\hline Rubber and plastics & 2.3 & 2.8 & 3.5 & 4.4 & 5.3 \\
\hline Nonnetallie products & 9.2 & 10.1 & 12.2 & 13.7 & 15.3 \\
\hline Metal products & 5.7 & 7.0 & 7.6 & 8.3 & 9.2 \\
\hline Melallie products & 3.4 & 4.1 & 4.0 & 4.1 & 4.4 \\
\hline Ptoducts made of metal & 2.3 & 2.9 & 3.6 & 4.2 & 4.8 \\
\hline Machinery and equipment & 1.4 & 1.6 & 1.7 & 2.2 & 2.7 \\
\hline Computer and offiee equipment & 0.0 & 0.0 & 0.0 & 0.2 & 0.3 \\
\hline Other machinery and equipment & 1.4 & 1.6 & 1.7 & 2.0 & 2.4 \\
\hline Electric and eketronic produets & 3.4 & 4.7 & 5.2 & 6.2 & 7.1 \\
\hline Radio, TV, telecom. equipment & 2.1 & 3.1 & 3.3 & 3.5 & 3.6 \\
\hline Medical instruments & 0.2 & 0.3 & 0.0 & 0.4 & 0.6 \\
\hline Other electric and electronic products & 1.1 & 1.3 & 1.7 & 2.3 & 2.9 \\
\hline Vehicles and transport oquipmint & 3.4 & 3.4 & 3.4 & 5.1 & 5.7 \\
\hline Anembling and repairing of mator wehicles & 1.5 & 1.4 & 1.6 & 1.7 & 1.8 \\
\hline Transport equiphent & 1.9 & 2.0 & 1.8 & 3.4 & 3.9 \\
\hline Furniture and other & 2.0 & 2.3 & 2.7 & 3.0 & 3.3 \\
\hline Furniture & 1.9 & 22 & 2.6 & 2.8 & 3.1 \\
\hline Other & 0.1 & 0.1 & 0.1 & 0.2 & 0.2 \\
\hline Slectricity, gas, and water & 6.2 & 7.3 & 8.5 & 9.4 & 10.3 \\
\hline Electricity and gas & 5.4 & 6.5 & 7.6 & 8.5 & 9.3 \\
\hline Water supply & 0.8 & 0.8 & 0.9 & 0.9 & 1.0 \\
\hline \multirow[t]{2}{*}{ Toial } & 103.3 & 118.1 & 334.4 & 151.2 & 167.0 \\
\hline & \multicolumn{5}{|c|}{ (Amnual percentage change) } \\
\hline Mining & 13.5 & 14.7 & 14.7 & 15.3 & 14.0 \\
\hline Oil and gas & 13.2 & 15.7 & 13.6 & 19.0 & 18.9 \\
\hline Mamufacturing & 13.5 & 13.9 & 13.5 & 12.2 & 9.9 \\
\hline Food and beverages & 12.9 & 14.4 & 10.0 & 7.4 & 7.9 \\
\hline Textiles and garments & 46.4 & 7.7 & 18.4 & 12.1 & 100 \\
\hline Metal products & $\ldots$ & 22.8 & 8.6 & 9.2 & 10.8 \\
\hline Electric and electronic products & $\ldots$ & 38.2 & 10.6 & 19.2 & 14.5 \\
\hline Vehicles and transport equipmenl & $\cdots$ & 0.0 & 0.0 & 50.0 & 11.8 \\
\hline Electricity, gas, and water & 18.5 & 18.5 & 15.0 & 11.8 & 9.6 \\
\hline Total & 14.5 & 14.2 & 13.8 & 12.5 & 10.4 \\
\hline
\end{tabular}

Soure: General Statistical Office.

1/ Due to reclassification of industrial activities, previously published industrial sector data are not comparable with the dats in this table. 
Table 9. Vietnam: Industrial Production by Sector of Ownership at Constant Prices, 1995-99 1/

\begin{tabular}{|c|c|c|c|c|c|}
\hline & 1995 & 1996 & 1997 & 1998 & $\begin{array}{r}1999 \\
\text { Estimate }\end{array}$ \\
\hline & \multicolumn{5}{|c|}{ (In billions of dong, at constant 1994 prices) } \\
\hline Total industrial production & 103,375 & 118,097 & 134,420 & 151,223 & 166.965 \\
\hline State sector & 51,991 & 58,166 & 64,474 & 69,462 & 72,604 \\
\hline (excluding oil and gas) & 41,146 & 45,699 & 50,235 & 52.593 & 52,538 \\
\hline Central & 33,920 & 38,411 & 42,215 & 45,677 & 47,986 \\
\hline (excluding oil and gas) & 23,075 & 25,944 & 27,976 & 28,808 & 27,920 \\
\hline Local & 18,071 & 19,755 & 22,259 & 23,785 & 24,618 \\
\hline Nonstate sector & 25,451 & 28,368 & 31,068 & 33,403 & 36,342 \\
\hline Cooperatives & 650 & 684 & 751 & 859 & $\ldots$ \\
\hline Private & 2,277 & 2,792 & 3,224 & 3,383 & $\cdots$ \\
\hline Household & 18,191 & 18,977 & 19,704 & 20,827 & $\cdots$ \\
\hline Mixed & 4,333 & 5,915 & 7,389 & 8,334 & $\ldots$ \\
\hline \multirow[t]{2}{*}{ Foreign invested sector } & 25,933 & 31,562 & 38.878 & 48,358 & 58,019 \\
\hline & \multicolumn{5}{|c|}{ (In percent of toual industrial production) } \\
\hline State sector & 50.3 & 49.3 & 48.0 & 45.9 & 43.5 \\
\hline (excluding oil and gas) & 39.8 & 38.7 & 37.4 & 34.8 & 31.5 \\
\hline Central & 22.3 & 22.0 & 20.8 & 19.1 & 16.7 \\
\hline (excluding oil and gas) & 17.5 & 16.7 & 16.6 & 15.7 & 14.7 \\
\hline Local & 24.6 & 24.0 & 23.1 & 22.1 & 21.8 \\
\hline Nenstate sector & 24.6 & 24.0 & 23.1 & 22.1 & 21.8 \\
\hline Cooperatives & 0.6 & 0.6 & 0.6 & 0.6 & $\ldots$ \\
\hline Private & 2.2 & 2.4 & 2.4 & 2.2 & $\cdots$ \\
\hline Household & 17.6 & 16.1 & 14.7 & 13.8 & $\ldots$ \\
\hline Mixed & 4.2 & 5.0 & 5.5 & 5.5 & ... \\
\hline \multirow[t]{2}{*}{ Foreign invested sector } & 25.1 & 26.7 & 28.9 & 32.0 & 34.7 \\
\hline & \multicolumn{5}{|c|}{ (Annual percentage change) } \\
\hline Totsi industrial production & 34.5 & 14.2 & 13.8 & 12.5 & 10.4 \\
\hline State sector & 13.6 & 11.9 & 10.8 & 7.7 & 4.5 \\
\hline (excluding oil and gas) & 13.8 & Ll.L & 9.9 & 4.7 & -0.2 \\
\hline Central & 14.0 & 13.2 & 9.9 & 8.2 & 5.1 \\
\hline (excluding oil and gas) & 14.4 & 12.4 & 7.8 & 3.0 & -3.1 \\
\hline Local & 12.6 & 9.3 & 12.7 & 6.9 & 3.5 \\
\hline Nonstate sector $2 f$ & 18.5 & 11.5 & 9.5 & 7.5 & 8.8 \\
\hline Cooperatives & $\cdots$ & 5.2 & 9.8 & 14.4 & $\ldots$ \\
\hline Private & $\cdots$ & 22.6 & 15.5 & 4.9 & $\ldots$ \\
\hline Household & $\ldots$ & 4.3 & 3.8 & 5.7 & $\ldots$ \\
\hline Mixed & $\cdots$ & 36.5 & 24.9 & 12.8 & $\ldots$ \\
\hline Foreign invested sector & $\ldots$ & 21.7 & 23.2 & 24.4 & 20.0 \\
\hline
\end{tabular}

Sources: General Statistical Office; and staff estimates.

1/ Due to reclassification of industrial activities, previously pubished industrial sector data are not comparable with the data in this table.

2/ Growth rate for 1995 covers both nonstate and foreign invested sectors. 
Table 10. Vietnam: Population and Employment, 1995-99

\begin{tabular}{|c|c|c|c|c|c|}
\hline & 1995 & 1996 & 1997 & 1998 & $\begin{array}{c}1999 \\
\text { Estinate }\end{array}$ \\
\hline & \multicolumn{5}{|c|}{ (In thousands of persons, unless otherwige indisated) } \\
\hline Total pophation U/ & $\mathbf{7 2 , 2 3 5}$ & 73,366 & 74,469 & 75,561 & 76.585 \\
\hline (annue) percethluge change) & 1.7 & 1.6 & 1.5 & 1.5 & 1.4 \\
\hline Urban $2 /$ & 14,928 & 15,547 & 15,323 & 17,197 & 18.165 \\
\hline (anmual percentage change) & 3.7 & 4.] & 5.0 & 5.3 & 5.6 \\
\hline Rusal $2 /$ & 57.306 & 57,819 & 58,146 & 58,365 & 58,420 \\
\hline (araual percentage change) & 1.1 & 0.9 & 0.6 & 0.4 & 0.1 \\
\hline Female & 37,103 & 37,591 & 38,059 & 38,511 & 38,908 \\
\hline Mala & 35,132 & 35,775 & 36,410 & 37,051 & 37,677 \\
\hline Age under 15 & $\ldots$ & $\ldots$ & $\cdots$ & $\ldots$ & 23,340 \\
\hline As $15-59$ & $\ldots$ & $\ldots$ & $\ldots$ & $\ldots$ & 46,302 \\
\hline Age 60 and over & $\ldots$ & $\ldots$ & $\ldots$ & $\ldots$ & 6,943 \\
\hline Total employnent & 34,590 & 35.792 & 36,994 & 37,877 & 38,546 \\
\hline \multirow[t]{2}{*}{ Unemployment rate $3 /$} & 5.8 & 5.9 & 6.0 & 6.9 & 7.4 \\
\hline & \multicolumn{5}{|c|}{ (In percent of total populdtion) } \\
\hline Urban $2 f$ & 20.7 & 21.2 & 21.9 & 228 & 23.7 \\
\hline Rural 2 & 793 & 78.8 & 78.1 & 77.2 & 76.3 \\
\hline Fenrate & 514 & 51.2 & 51.1 & 51.0 & 50.8 \\
\hline Male & 48.6 & 48.8 & 48.9 & 49.0 & 49.2 \\
\hline Age under 15 & +. & $\ldots$ & $\ldots$ & $\cdots$ & 30.5 \\
\hline Age $15-59$ & $\ldots$ & $\ldots$ & $\ldots$ & $m$ & 60.5 \\
\hline Age 60 and ower & $\ldots$ & $\ldots$ & $\ldots$ & $\ldots$ & 9.1 \\
\hline \multirow[t]{2}{*}{ Total employment } & 47.9 & 48,8 & 49.7 & 501 & 50.5 \\
\hline & \multicolumn{5}{|c|}{ (In thousands of pefsons) } \\
\hline Tothi emplinyment & 34,590 & 35,792 & 36,994 & 37.87 & 38,546 \\
\hline Nonstate sector & $3], 537$ & 32,654 & 33,727 & 34,494 & 35.176 \\
\hline State sector & 3.053 & 3,138 & 3.267 & 3,383 & 3,370 \\
\hline Government & 1,258 & 1,278 & $\ldots$ & $\ldots$ & $\cdots$ \\
\hline Central & 251 & 201 & $\ldots$ & $\ldots$ & $\ldots$ \\
\hline Local & 997 & 1,077 & ... & $\ldots$ & $\cdots$ \\
\hline State enterprizes & 1,795 & 1,860 & -. & $\ldots$ & $\ldots$ \\
\hline Central & 1,001 & 1,084 & $\ldots$ & $\ldots$ & $\cdots$ \\
\hline \multirow[t]{2}{*}{ local } & 794 & 776 & $\cdots$ & $\ldots$ & $\cdots$ \\
\hline & \multicolumn{5}{|c|}{ (In percent of total employiment) } \\
\hline Nonstate sector & 91.2 & 91.2 & 91.2 & 91.1 & 91.3 \\
\hline Slate sector & 8.8 & 8.8 & 8.8 & 8.9 & 8.7 \\
\hline Government & 3.6 & 3.6 & $\ldots$ & $\ldots$ & $\ldots$ \\
\hline Central & 0.8 & 0.6 & $\cdots$ & $\cdots$ & $\cdots$ \\
\hline Local & 29 & 3.0 & $\cdots$ & $\cdots$ & $\ldots$ \\
\hline State enterprises & 5.2 & 5.2 & $\ldots$ & $\ldots$ & .. \\
\hline Centual & 2.9 & 3.0 & $\ldots$ & $\ldots$ & $\ldots$ \\
\hline \multirow[t]{2}{*}{ Ioeal } & 23 & 27 & $\ldots$ & $\ldots$ & $\ldots$ \\
\hline & \multicolumn{5}{|c|}{ (Annual percentage change) } \\
\hline Total employment & 2.7 & 3.5 & 3.4 & 24 & 18 \\
\hline Nonstate sectior & 2.6 & 3.5 & 3.3 & 2.3 & 2.0 \\
\hline State sector & 4.3 & 2.8 & 4.1 & 3.6 & -0.4 \\
\hline Government & 6.8 & 1.6 & $\ldots$ & $\ldots$ & $\ldots$ \\
\hline Centra] & 1.6 & -23.0 & $\ldots$ & $\ldots$ & $\ldots$ \\
\hline Local & 8.3 & 8.0 & $\ldots$ & $\ldots$ & ... \\
\hline State enterprises & 2.6 & 3.6 & $\cdots$ & $\cdots$ & $\cdots$ \\
\hline Central & 1.4 & 8.3 & $\ldots$ & $\cdots$ & ... \\
\hline Local & 4,0 & -2.3 & $\cdots$ & $\therefore$ & $\ldots$ \\
\hline
\end{tabular}

Sources: Generat Statistical Office; and staff estimates.

W Annual official estimstes adjusted on the basis of the 1989 and 1999 ccnsus.

2 Armed forces and some other special proups arc excluded from utbentural ategories

3 For labor force comprising males 15 to 60 years old and females $1.5-55$ years old

\section{CInternational Monetary Fund. Not for Redistribution}


Table I1. Vietrum: Total and Nonstate Bmployment by Sector, $1998-99$

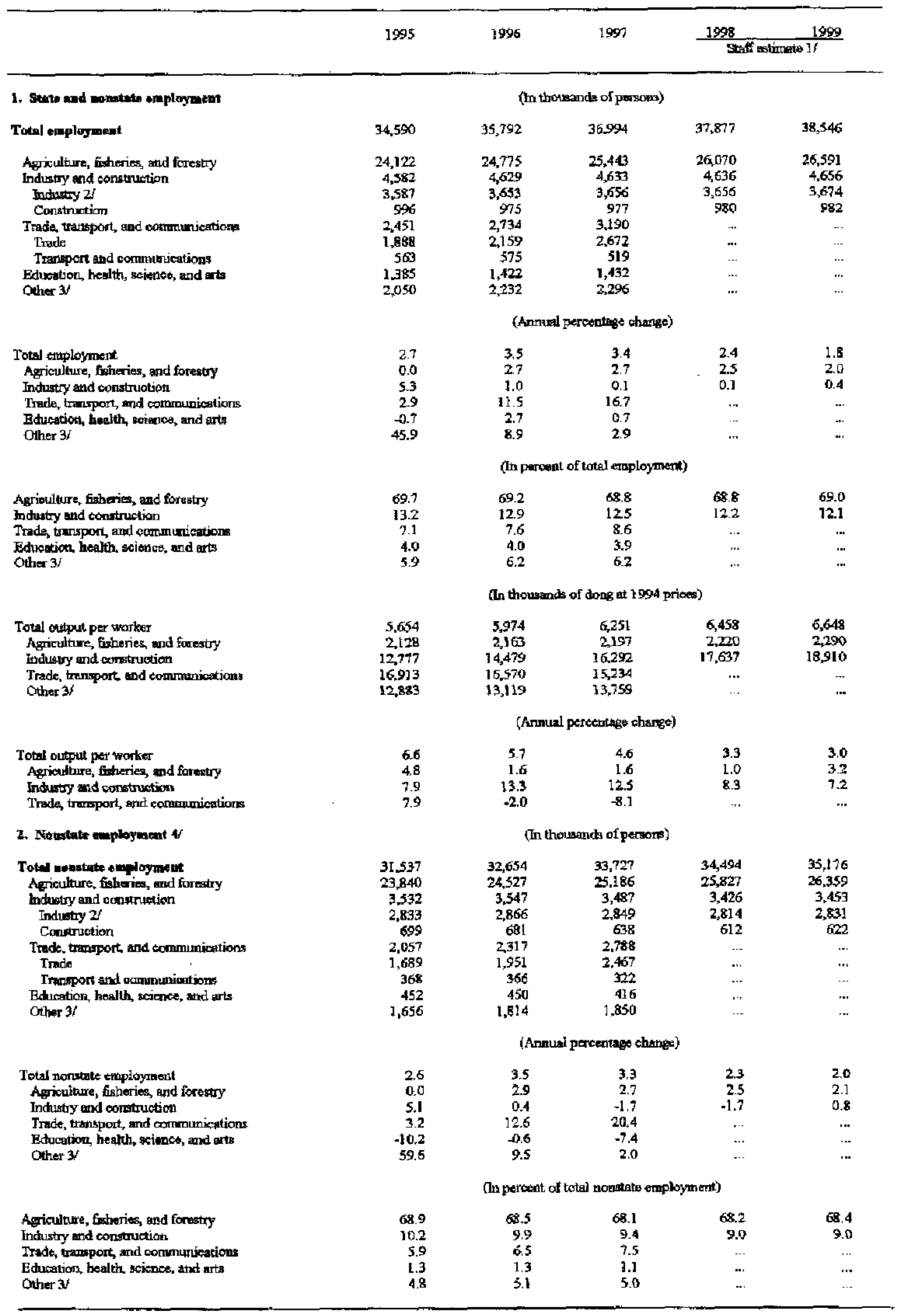

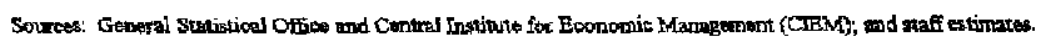

$1 /$ Rased on estimates of total employment in National Accownting Framework and a ldacraccomometric Model for Vietmum (2000, CEML.

2/ Iroludes mining and quarying and electricity, gan, and water supiy.

$3 /$ Includes unclassified workers.

4/ Emptoyment outside centril and local goremmonl and state-owned entepritas. 
Table 12. Viethum: Employment b the Strte Sector, 1995-95

\begin{tabular}{|c|c|c|c|c|c|}
\hline & 1995 & 1996 & 1997 & 1998 & $\begin{array}{c}1999 \\
\text { Eistimnate }\end{array}$ \\
\hline & \multicolumn{5}{|c|}{ (In thousands of persons) } \\
\hline Total employmeat & 3,053 & 3,138 & 3,267 & 3,383 & 3,370 \\
\hline Agriculture and forestry & 282 & 248 & 257 & 243 & 232 \\
\hline Industy and constrixtion & 1,051 & 1,082 & 1,146 & 1,210 & 1,203 \\
\hline Industry & $7 \$ 4$ & 787 & 807 & 842 & 843 \\
\hline Construction & 297 & 294 & 338 & 368 & 360 \\
\hline Trade, transport, and communications & 393 & 417 & 402 & 400 & 391 \\
\hline Trade & 198 & 208 & 205 & 201 & 192 \\
\hline Tramsport and communicatiots & 195 & 210 & 197 & 199 & 199 \\
\hline Education, health, sciences, and arts & 933 & 973 & 1,016 & 1,070 & 1,080 \\
\hline Education & $71 I$ & 741 & $\pi 7$ & 818 & 879 \\
\hline Science & 27 & 32 & 32 & 37 & 34 \\
\hline Culture, arts, and sport & 32 & 32 & 33 & 35 & 34 \\
\hline Public bealth & 163 & 368 & 173 & 180 & 183 \\
\hline \multirow[t]{2}{*}{ Other } & 394 & 418 & 447 & 450 & 464 \\
\hline & \multicolumn{5}{|c|}{ (In percent of botal state sector employment) } \\
\hline Afrietliture and forestry & 9.2 & 7.9 & 7.9 & 7.2 & 69 \\
\hline Industry and construction & 34.4 & 34.5 & 35.1 & 35.8 & 35.7 \\
\hline Trabs thophot, and conmunications & 12.9 & 13.3 & 12.3 & 11.8 & 11.6 \\
\hline Extucetion health seience, and arts & 30.5 & 31.0 & 31.1 & 31.6 & 32.0 \\
\hline \multirow[t]{2}{*}{ Other } & 12.9 & 13.3 & 13.7 & 13.6 & 13.8 \\
\hline & \multicolumn{5}{|c|}{ (Annuai persentage change) } \\
\hline Total employment & 4.3 & 2.8 & 4.1 & 3.6 & 0.4 \\
\hline Agriculture and forestry & -2.3 & +121 & 3.6 & .5 .4 & 4.5 \\
\hline Industry and construction & 6.0 & 2.9 & 5.9 & 5.6 & -0.6 \\
\hline Trade, transport, and fornmunications & 1.0 & 6.0 & .3 .6 & -0.5 & -2.3 \\
\hline Education, health, science, and arts & 4.7 & 43 & 4.4 & 5.4 & 0.9 \\
\hline \multirow[t]{2}{*}{ Other } & 7.2 & 6.2 & 6.8 & 3.0 & 09 \\
\hline & \multicolumn{5}{|c|}{ (to thousands of dong per month) } \\
\hline Average noming I wage I/ & 478 & 543 & 642 & 697 & 698 \\
\hline Inciustry & 754 & 708 & BA+1 & 898 & $8 B 9$ \\
\hline Construction & 499 & 573 & 738 & 807 & 787 \\
\hline Agriculture & 366 & 422 & 480 & 514 & 493 \\
\hline Transportation & 879 & L,018 & 1,132 & 1,304 & 1,322 \\
\hline Trade & 490 & 582 & 688 & 742 & 301 \\
\hline Educekion & 310 & 329 & 405 & 451 & 488 \\
\hline Sciente & 361 & 505 & 555 & 673 & 543 \\
\hline Culture, atts, and sponts & 347 & 400 & 453 & 540 & 495 \\
\hline Public heslth & 327 & 363 & 440 & 480 & 516 \\
\hline State management & 357 & 380 & 435 & 449 & 463 \\
\hline Financial intermedialion & 807 & 940 & $\ldots$ & $\cdots$ & $\cdots$ \\
\hline Average real wage growth 2 f & 4.8 & 7.6 & 14.7 & 0.6 & -3.8 \\
\hline Intustry & 120 & -11.0 & 15.2 & -1.0 & -4.9 \\
\hline Construction & 2.3 & 8.6 & 25.1 & 1.3 & -6.3 \\
\hline Aqriculiure & 9.3 & 9.0 & 10.4 & -0.7 & -7.9 \\
\hline Transpoctation & 35.7 & 9.7 & 7.8 & 6.8 & -2.5 \\
\hline Trede & 4.0 & 124 & 14.7 & 0.0 & -9.3 \\
\hline Edueation & -9.8 & 0.5 & 19.6 & 3.2 & 3.9 \\
\hline Seitatare & -11.6 & 32.4 & 6.5 & 12.5 & -22.5 \\
\hline Culture, arts, and sparts & 4.4 & 9.1 & 9.9 & 10.4 & -12.0 \\
\hline Public health & -6.7 & 5.1 & 17.5 & 1,2 & 3.2 \\
\hline State managernent & -6.9 & 0.8 & 11.2 & -4.4 & -1.0 \\
\hline Finnancial intermediation & 1.3 & 103 & $\cdots$ & & $\ldots$ \\
\hline Consumer price inflation & 16.9 & 5.6 & 3.1 & 7.9 & 4.1 \\
\hline
\end{tabular}

Soures: Gencral Statistical Office; and staff extimates.

1/ Cash income, includinq payments in kind, bonus payments, and social security contributions. 2/ Nominal wage growth deflated by consumer price inflation. 
Table 13. Vhethada: Summary ar General Government Budgatary Operations, 1995-200fi $u$

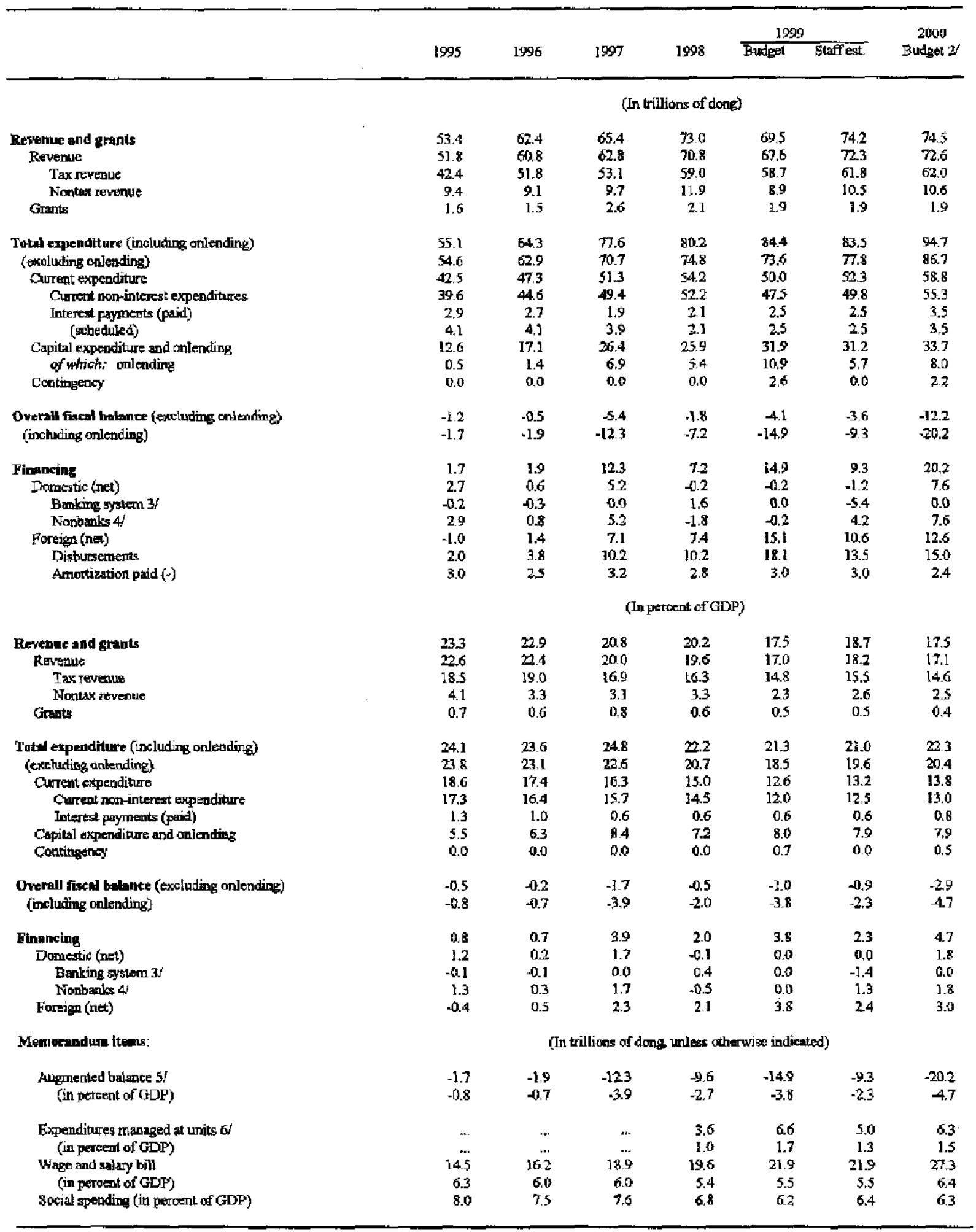

Sources: Ministry of Finanoe; and staff estimates and projections.

1/Cuh bagis.

If Excludes cument and capital costs of state-owaed enterprise and bankinis sextor reforms.

3/ The difference between bank financing and net claims on government in the monetary survey for l998 is gut to bank recapjtalization operations. 4/ Domestic nonbunk finaraing is derived as a residual and includes statistical discrepency.

$5 /$ Includes capital costs of brik restruching estimated at D 2.4 tribion (0.7 percent of GDP) in 1998 .

6' Self-financed expenditure undertaken by administrative units of the central and local goveraments, which are cxceluded frome the above budget presentation 
Table 14. Vietnam; Government Revenues, 1995-2000

\begin{tabular}{|c|c|c|c|c|c|c|c|}
\hline \multirow[b]{4}{*}{ Total revenue and grants } & \multirow{2}{*}{1995} & \multirow[t]{2}{*}{1996} & \multirow[t]{2}{*}{1997} & \multirow[t]{2}{*}{1998} & \multicolumn{2}{|c|}{1999} & \multirow{2}{*}{$\begin{array}{l}2000 \\
\text { Budget }\end{array}$} \\
\hline & & & & & Budget & $\begin{array}{r}\text { Staff } \\
\text { estimate }\end{array}$ & \\
\hline & \multicolumn{6}{|c|}{ (In trillions of dong) } & \\
\hline & $\$ 3.4$ & 62.4 & 65.4 & 73.0 & 69.5 & 74.2 & 74.5 \\
\hline Tax revenue & 42.4 & 51.8 & 53.1 & 59.0 & 58.7 & 61.8 & 62.0 \\
\hline Corporate income tax 1 & 7.4 & 9.6 & 11.6 & 13.1 & 12.3 & 14.0 & 15.2 \\
\hline Individual income tax & 0,5 & 1.4 & 1.5 & 1.8 & 1.5 & 1.9 & 1.6 \\
\hline Capitsl user charge & 1.3 & 1.5 & 1.5 & 1.7 & L.3 & 1.5 & 1.4 \\
\hline Land and housing tax & 0.3 & 0.4 & 0.3 & 0.3 & 0.3 & 0.3 & 0.3 \\
\hline License tax & 0.2 & 0.2 & 0.4 & 0.3 & 0.4 & 0,4 & 0.4 \\
\hline Tax on the transfer of properties & $\ldots$ & $\ldots$ & 1.0 & 1.0 & 1.0 & 1.0 & 0.8 \\
\hline Tax on land use right & $\ldots$ & $\ldots$ & 0.3 & 0.4 & 0.3 & 0.3 & 0.2 \\
\hline Value added $\operatorname{tax}$ (VAT) $2 /$ & 7.8 & 10.1 & 11.8 & 11.8 & 14.0 & 15.0 & 15.4 \\
\hline Excises & 2.5 & 3.2 & 4.6 & 5.6 & 4.4 & 4.5 & 4.5 \\
\hline Slaughter tax & 0.1 & 0.1 & 0.1 & 0.0 & 0.0 & 0.0 & 0.0 \\
\hline Natural resources tax & 2.4 & 3.0 & 3.4 & 3.3 & 3.7 & 4.3 & 4.8 \\
\hline Agricultural tax & 1.6 & 1.9 & 1.7 & 2.0 & 1.8 & 2.0 & 1.7 \\
\hline Taxes on trade & 13.3 & 35.1 & 13.5 & 14.9 & 15.5 & 14.5 & 15.0 \\
\hline Petroleuni surcharge & 0.0 & 0,0 & 0.0 & 1.5 & 1.5 & 1.0 & 0.2 \\
\hline Other taxes & 4.9 & 5.3 & 1.4 & 1.4 & 0.5 & 1.2 & 0.5 \\
\hline Nontax revenue & 9.4 & 9.1 & 9.7 & 11.9 & 8.9 & 10.5 & 10.6 \\
\hline Fees and charges & $\ldots$ & $\ldots$ & 3.9 & 4.1 & 4.0 & 3.9 & 4.0 \\
\hline Rentil of land & $\ldots$ & $\ldots$ & 0.5 & 0.5 & 0.5 & 0.6 & 0.5 \\
\hline Net profit after tax & $\ldots$ & $\cdots$ & 2.4 & 2.1 & 2.5 & 2.9 & 4.2 \\
\hline Capital revetules & $\ldots$ & $\ldots$ & 0.8 & 0.8 & 0.5 & 0.8 & 0.4 \\
\hline Other & $\cdots$ & 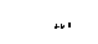 & 2.1 & 4.3 & 1.4 & 2.4 & 1.6 \\
\hline \multirow[t]{2}{*}{ Grants } & 1.6 & 1.5 & 2.6 & 2.1 & 1.9 & 1.9 & 1.9 \\
\hline & \multicolumn{7}{|c|}{ (In percent of GDP) } \\
\hline Total revenue and grants & 23.3 & 22.9 & 20.8 & 20.2 & 17.5 & 18.7 & 17.5 \\
\hline Tax revenue & 18.5 & 19.0 & 16.9 & 16.3 & 14.8 & 15.5 & 14.6 \\
\hline Corporate income tax $1 /$ & 3.3 & 3.5 & 3.7 & 3.6 & 3.1 & 3.5 & 3.6 \\
\hline Individual income tax & 0.2 & 0.5 & 0.5 & 0.5 & 0.4 & 0.5 & 0.4 \\
\hline Capital user charge & 0.6 & 0.6 & 0.5 & 0.5 & 0.3 & 0.4 & 0.3 \\
\hline Land and housing tax & 0.1 & 0.1 & 0.1 & 0.1 & 0.1 & 0.1 & 0.1 \\
\hline License tax & 0.1 & 0.1 & 0.1 & 0.1 & 0.1 & 0.1 & 0.1 \\
\hline Tax on the transfer of properties & $\ldots$ & $\ldots$ & 0.3 & 0.3 & 0.3 & 0.3 & 0.2 \\
\hline Tax on land use right & $\ldots$ & $\ldots$ & 0.1 & 0.1 & 0.1 & 0.1 & 0.1 \\
\hline VAT 2I & 3.4 & 3.7 & 3.8 & 3.3 & 3.5 & 3.8 & 3.6 \\
\hline Excises & 1.1 & 1.2 & 1.5 & 1.5 & 1.1 & 1.1 & 1.1 \\
\hline Slaughter tax & 0.0 & 0,0 & 0.0 & 0.0 & 0,0 & 0.0 & 0.0 \\
\hline Natural resources tax & 1.0 & 1.1 & 1.1 & 0.9 & 0.9 & 1,1 & 1.1 \\
\hline Agricultural tax & 0.7 & 0.7 & 0.5 & 0.5 & 0.5 & 0.5 & 0.4 \\
\hline Taxes on trade & 5.8 & 5.6 & 4.3 & 4.1 & 3.9 & 3.6 & 3.5 \\
\hline Petroleum sureharge & 0.0 & 0.0 & 0.0 & 0.4 & 0.4 & 0.2 & 0.0 \\
\hline Other taxes & 2.1 & 1.9 & 0.4 & 0.4 & 0.1 & 0.3 & 0.1 \\
\hline Nontax reverue & 4.1 & $\mathbf{3 . 3}$ & 3.1 & 3.3 & 2.3 & 2.6 & 2.5 \\
\hline Fees and charges & $\ldots$ & $\ldots$ & 1.2 & 1.1 & 1.0 & 1.0 & 0.9 \\
\hline Rental of land & $\ldots$ & $\ldots$ & 0.1 & 0.2 & 0.1 & 0.1 & 0.1 \\
\hline Net profit after tax & $\ldots$ & $\ldots$ & 0.8 & 0.6 & 0.6 & 0.7 & 1.0 \\
\hline Capital revenues & $\cdots$ & $\ldots$ & 0,3 & 0.2 & 0.1 & 0.2 & 0.1 \\
\hline Other & $\cdots$ & $\ldots$ & 0.7 & 1.2 & 0.4 & 0.6 & 0.4 \\
\hline Grants & 0.7 & 0.6 & 0.8 & 0.6 & 0.5 & 0.5 & 0.4 \\
\hline
\end{tabular}

Sources: Ministry of Finance, Budget Department; and staff extimates.

1/ Profit tax prior to 1999.

2/ Turnowe tax prior to 1999. 
Table 15. Vietinam: Govemment Expenditures, 1995-2000

\begin{tabular}{|c|c|c|c|c|c|c|c|}
\hline & \multirow[t]{2}{*}{1995} & \multirow[t]{2}{*}{1996} & \multirow{2}{*}{1997} & \multirow[t]{2}{*}{1998} & \multicolumn{2}{|c|}{1999} & \multirow{2}{*}{$\begin{array}{c}2000 \\
\text { Budget } 1 /\end{array}$} \\
\hline & & & & & Budget & $\begin{array}{r}\text { Staff } \\
\text { estimate }\end{array}$ & \\
\hline & \multicolumn{7}{|c|}{ (In trillions of dong) } \\
\hline Total expenditure (cash basis) & 55.1 & 64.3 & 77.6 & 80.2 & 84.4 & 83.5 & 94.7 \\
\hline Total current expenditure (cash basis) & 42.5 & 47.3 & 51.3 & $\$ 4,2$ & 50.0 & 52.3 & 98.8 \\
\hline General administrative services & 5.7 & 6.4 & 7.1 & 6.7 & 4.8 & 5.8 & 5.2 \\
\hline Econonnic services & 4.0 & 4.2 & 4.5 & 4.8 & 4.8 & 5.? & 5.1 \\
\hline Social services & 18.2 & 20.3 & 23.7 & 24.4 & 24.7 & 25.4 & 26.7 \\
\hline Education & 4.7 & 5.5 & 7.2 & 7.7 & 7.9 & 8.0 & 8.8 \\
\hline Health & 2.4 & 2.8 & 3.0 & 3.1 & 2.9 & 3.1 & 3.3 \\
\hline Sooial subsidies & 7.4 & 8.2 & 9.2 & 8.7 & 8.9 & 9.1 & 8.8 \\
\hline Other & 3.8 & 3.9 & 4.3 & 5.0 & 5.0 & $\$ .2$ & 5.7 \\
\hline Other novinterest expenditure $2 /$ & 11.7 & 13.7 & 14.0 & 16.2 & 13.2 & 13.5 & 18,2 \\
\hline Interest payments (paid) & 4.1 & 4.1 & 3,9 & 2.1 & 2.5 & 2.5 & 3.5 \\
\hline Foreign & 2.6 & 2.6 & 2.9 & 0.9 & $\cdots$ & $\cdots$ & $\cdots$ \\
\hline Domestic & 1.5 & 1.5 & 1.0 & 1.2 & $\cdots$ & $\ldots$ & $\cdots$ \\
\hline Capital expenditure and ondending & 12.6 & 17.1 & 26.4 & 25.9 & 31.9 & 31.2 & 33.7 \\
\hline Capital expenditure & 12.1 & 15.6 & 19.5 & 20.5 & 21.0 & 25.5 & 25.7 \\
\hline Onlending & 0.5 & 1.4 & 6.9 & 5.4 & 10.9 & 5.7 & 8.0 \\
\hline \multirow[t]{2}{*}{ Contingency } & 0.0 & 0.0 & 0.0 & 0.0 & 2.6 & 0.0 & 2.2 \\
\hline & \multicolumn{7}{|c|}{ (In percent of GDP) } \\
\hline Total expenditure (casti basis) & 24.1 & 23.6 & 24.8 & 22.2 & 21.3 & 21.6 & 22.3 \\
\hline Total current expenditure (cash basis) & 18.6 & 17.4 & 16.3 & 15.0 & 12.6 & 13.2 & 13.8 \\
\hline General administrative services & 2.5 & 2.3 & 2.3 & 1.9 & 1.2 & 1.5 & 1.2 \\
\hline Economic services & 1.7 & 1.5 & 1.4 & 1.3 & 1.2 & 1,3 & 1.2 \\
\hline Social services & 8.0 & 7.5 & 7.6 & 6.8 & 6.2 & 6.4 & 6.3 \\
\hline Educiation & 2.1 & 2.0 & 23 & 2.1 & 2.0 & 2.0 & 2.1 \\
\hline Health & 1.0 & 1.0 & 1.0 & 0.8 & 0.7 & 0.8 & 0.8 \\
\hline Social stbsidies & 3.2 & 3.0 & 2.9 & 2.4 & 2.2 & 2.3 & 2.1 \\
\hline Other & 1.6 & 1.4 & 1.4 & 1.4 & 1.3 & 1.3 & 1.4 \\
\hline Other nonimterest expendialre 2 & 5.1 & 5.0 & 4.5 & 4.5 & 3.3 & 3.4 & 4.3 \\
\hline Interest payments (paid) & 1.8 & 1.5 & 1.2 & 0.6 & 0.6 & 0.6 & 0.8 \\
\hline Foreign & 1.1 & 1.0 & 0.9 & 0.2 & -.. & $\ldots$ & $\ldots$ \\
\hline Domestic & 0.7 & 0.6 & 0.3 & 0.3 & $\cdots$ & $\ldots$ & $\cdots$ \\
\hline Capital expenditure and onlending & 9.5 & 6.3 & 8.4 & 7.2 & 8.0 & 7.9 & 7.9 \\
\hline Capital expenditure & $\$ .3$ & 5.7 & 6.2 & 5.7 & 5.3 & 6.4 & 6.1 \\
\hline Onlending & 0.2 & 0.5 & 2.2 & 1.5 & 2.7 & 1.4 & 1.9 \\
\hline Contingency & 0.0 & 0.0 & 0.0 & 0.0 & 0.7 & 0.0 & 0.5 \\
\hline
\end{tabular}

Sources: Ministry of Finance, Budget Department; and staff estimates.

1. Excludes current and capital cests of state-owned enterprise and banking sector reforms.

2 Includes defense expenditures. 
Table 16. Vienam: Treasury Bill and Bond Issues, 1996-2000 1/

(In billions of dong, unless otherwise indicated)

\begin{tabular}{|c|c|c|c|c|c|c|c|c|}
\hline \multirow[b]{2}{*}{ Auction date } & \multicolumn{3}{|c|}{ Ireasury Bills (one-year) } & \multicolumn{3}{|c|}{ Treasury Bands (two-year) } & \multicolumn{2}{|c|}{ Treasury Bonds (five-year) } \\
\hline & $\begin{array}{l}\text { Amount } \\
\text { sold }\end{array}$ & $\begin{array}{l}\text { Average } \\
\text { yield }\end{array}$ & $\begin{array}{l}\text { End-month } \\
\text { outstanding stock }\end{array}$ & $\begin{array}{l}\text { Amount } \\
\text { sold }\end{array}$ & $\begin{array}{l}\text { Average } \\
\text { yeeld }\end{array}$ & $\begin{array}{l}\text { End-month } \\
\text { outstanding slock }\end{array}$ & $\begin{array}{l}\text { Amount } \\
\text { sold }\end{array}$ & $\begin{array}{l}\text { End-month } \\
\text { outstanding stoek }\end{array}$ \\
\hline 1996 December & 0 & $\ldots$ & 824 & 92 & 12.0 & 1,260 & $\ldots$ & $\ldots$ \\
\hline 1997 January & 0 & $\ldots$ & 824 & 0 & $\ldots$ & 1,260 & $\ldots$ & $\ldots$ \\
\hline February & 60 & 11.3 & 884 & 322 & 14.0 & 1,582 & $\ldots$ & $\ldots$ \\
\hline Manch & 118 & 11.7 & 1,002 & 554 & 14.0 & 2,136 & $\ldots$ & $\ldots$ \\
\hline April & 1 & 12.0 & 1,003 & 460 & 14.0 & 2,596 & $\ldots$ & $\cdots$ \\
\hline May & 200 & 12.0 & 1,203 & 212 & 14.0 & 2,808 & $\cdots$ & $\cdots$ \\
\hline June & 340 & 11.8 & 1,443 & 179 & 14.0 & 2,987 & $\ldots$ & $\cdots$ \\
\hline July & 548 & 11.2 & 1,872 & 388 & 14.0 & 3,375 & $\cdots$ & $\ldots$ \\
\hline August & 253 & 10.9 & 1,825 & 469 & 14.0 & 3,844 & $\ldots$ & $\cdots$ \\
\hline September & 521 & 10.3 & 2,225 & 345 & 120 & 4,189 & $\cdots$ & $\ldots$ \\
\hline Octcber & 671 & 9.5 & 2,729 & 316 & 12.0 & 4,504 & $\cdots$ & $\ldots$ \\
\hline November & 202 & 9.3 & 2915 & 200 & 12.0 & 4,704 & $\ldots$ & $\ldots$ \\
\hline December & 3 & 9.0 & 2,918 & 132 & 12.0 & 4,836 & $\ldots$ & $\ldots$ \\
\hline 1998 January & 0 & $\ldots$ & 2,918 & 0 & $\ldots$ & 4,836 & ... & $\ldots$ \\
\hline February & 25 & 113 & 2,882 & 0 & $\ldots$ & 4,836 & $\ldots$ & $\cdots$ \\
\hline March & 33 & 12.0 & 2,797 & 185 & 12.0 & 5,021 & $\ldots$ & $\cdots$ \\
\hline April & 184 & 11.7 & 2,980 & 172 & 13.0 & 5,193 & $\ldots$ & $\ldots$ \\
\hline May & 378 & 11.8 & 3,158 & 232 & 13.0 & 5,424 & $\ldots$ & $\ldots$ \\
\hline June & 370 & 11.5 & 3,187 & 142 & 13.0 & 5,566 & $\ldots$ & $\ldots$ \\
\hline July & 529 & 11.7 & 3,158 & 147 & 13.0 & 5,713 & $\ldots$ & $\ldots$ \\
\hline August & 688 & 11.7 & 3,603 & 109 & 13.0 & 5,822 & $\cdots$ & $\cdots$ \\
\hline September & 396 & 11.7 & 3,478 & 615 & 14.0 & 5,896 & $\ldots$ & -. \\
\hline October & 550 & 11.6 & 3,357 & 559 & 14.0 & 5,996 & $\ldots$ & ... \\
\hline November & 200 & 11.6 & 3,355 & 410 & 14.0 & 6,238 & $\ldots$ & ... \\
\hline December & 369 & 11.6 & 3,721 & 272 & 13.0 & 6,418 & $\ldots$ & $\cdots$ \\
\hline 1999 January & 66 & 11.5 & 3,787 & 0 & $\ldots$ & 6,418 & $\ldots$ & $\ldots$ \\
\hline Februaty & 69 & 11.5 & 3,831 & 587 & 13.0 & 6,683 & $\ldots$ & $\cdots$ \\
\hline March & 255 & 11.0 & 4,053 & 1,366 & 13.0 & 7,495 & $\ldots$ & $\ldots$ \\
\hline April & 278 & 10.2 & 4,147 & 874 & 13.0 & 7,909 & $\ldots$ & $\ldots$ \\
\hline May & 201 & 9.9 & 3,970 & 341 & 13.0 & 8,037 & 1,507 & 1,507 \\
\hline June & 380 & 9.6 & 3,980 & 0 & $\ldots$ & 7,858 & 1,958 & 3,465 \\
\hline July & 324 & 9.4 & 3,775 & 0 & $\ldots$ & 7,470 & 1,031 & 4,496 \\
\hline August & 297 & 9.0 & 3,384 & 0 & $\ldots$ & 7,002 & 0 & 4,496 \\
\hline September & 410 & 7.9 & 3,398 & 0 & $\ldots$ & 6,657 & 0 & 4,496 \\
\hline October & 400 & 6.5 & 3,248 & 0 & $\ldots$ & 6,341 & 0 & 4,496 \\
\hline November & 333 & 4.9 & 3,381 & 0 & $\ldots$ & 6,141 & 0 & 4,496 \\
\hline December & 0 & 0.0 & 3,012 & 0 & $\ldots$ & 6,009 & $\mathbf{0}$ & 4,496 \\
\hline 2000 January & 325 & 6.0 & 3,271 & 0 & $\cdots$ & 6,009 & 0 & 4,496 \\
\hline February & 350 & 6.0 & 3,552 & 0 & $\ldots$ & 6,009 & 0 & 4,496 \\
\hline March & 977 & 5.7 & 4,274 & 0 & $\cdots$ & 5,824 & 0 & 4,496 \\
\hline \multicolumn{4}{|c|}{ Memorandum items: } & 1996 & 1997 & $\underline{1998}$ & 1999 & $20002 /$ \\
\hline \multirow{2}{*}{\multicolumn{4}{|c|}{$\begin{array}{l}\text { Total stock of debt (in trillions of dong, end of period) } \\
\text { (in percent of GDP) }\end{array}$}} & 2,084 & 7,754 & 10,139 & 13,517 & 14,595 \\
\hline & & & & 0.8 & 2.5 & 2.8 & 3.4 & 3.4 \\
\hline
\end{tabular}

Sources: State Bank of Vietnam and Ministry of Finance; and staff estimates.

1/ In addition to these amounts, D 246 billion in shorter-term maturity bills were anctioned during 1995-96. Treasury bills of varying maturities have also been placed directly with the public through the Ministry of Finance's retail network.

2/As of end-March 2000. 
Table 17. Vetnam; Monetary Survey, 1995-2000 1/

\begin{tabular}{|c|c|c|c|c|c|c|c|c|c|}
\hline & \multirow[t]{2}{*}{1995} & \multirow[t]{2}{*}{1996} & \multirow[t]{2}{*}{1997} & \multirow[t]{2}{*}{1998} & \multicolumn{4}{|c|}{1999} & \multirow{2}{*}{$\begin{array}{l}2000 \\
\text { March }\end{array}$} \\
\hline & & & & & Mrrch & June & Sqpt. & Dec. & \\
\hline & \multicolumn{9}{|c|}{ (In trillions of dong, end of period) } \\
\hline Net foreign arsets & 10.9 & 14.3 & 21.0 & 31.2 & 36.6 & 42.7 & 49.8 & 61.6 & 72.6 \\
\hline Foreign assets & 24.7 & 31.2 & 37.9 & 47.0 & 51.3 & 55.8 & 627 & 74.7 & 85.9 \\
\hline Foreign liabilities & -13.9 & -17.0 & -16.9 & -15.8 & .14 .7 & .13 .1 & -129 & -13.0 & -13.3 \\
\hline Net domertic assets & 41.9 & 50.4 & 60.6 & 71.2 & 71.0 & 70.3 & 71.2 & 81.0 & 84.1 \\
\hline Donuestic credit (net) & 47.1 & $\$ 5.3$ & 65.8 & $\mathbf{8} 1,0$ & 827 & 82.5 & 83.3 & 89,6 & 93.3 \\
\hline Net claims on govemment 2 & 4.7 & 4.4 & 4.4 & 8.4 & 6.8 & 4.6 & 4.1 & 2.9 & -1.7 \\
\hline Credit to the econonay & 42.4 & 50.9 & 62.4 & 72.7 & 75.9 & $\pi 7.9$ & 79.2 & 85.6 & 94.9 \\
\hline Claims on state enterpríses & 24.1 & 26.8 & 31.0 & 38.1 & 39.6 & 39.8 & 38.4 & 41.8 & 45.3 \\
\hline Claims on other sectors & 18.3 & 24.1 & 31.4 & 34.6 & 36.3 & 38.2 & 40.8 & 44.9 & 49.7 \\
\hline Other itens, net & -5.2 & -4.9 & -6.2 & -9.8 & -11.7 & -12.3 & -12.0 & -8.5 & -9.2 \\
\hline Broad money & 52.7 & 64.7 & $\mathbf{8 1 . 6}$ & 102.4 & 107.6 & 112.9 & 121.0 & 142.6 & 156.7 \\
\hline of which: total deposits & 33.5 & 42,0 & 56.5 & 75.5 & 81.1 & 87.2 & 94.0 & 101.1 & 113.8 \\
\hline Dong liquidity & 41.6 & 51.5 & 62.9 & 78.3 & 80.4 & 83.1 & 88.7 & 105.4 & 114.4 \\
\hline Currency outside banks & 19,2 & 22.6 & 25.1 & 27.0 & 26.5 & 25.7 & 27.0 & 41.5 & 42.9 \\
\hline Deposits & 22.5 & 28.9 & 37.8 & $\$ 1.4$ & 53,9 & 57.4 & 61.7 & 63.9 & 71.5 \\
\hline Demand deposits & 7.4 & 10.8 & 14.9 & 20.2 & 19.1 & 20.1 & 22.8 & 25.6 & 27.1 \\
\hline Other deposits & 15.1 & 18.1 & 22.9 & 31.1 & 34.8 & 37.3 & 38.9 & 38.3 & 44.4 \\
\hline Foreigh currenoy depositg & 11.1 & 13.2 & 18.7 & 24.1 & 27.2 & 29.9 & 32.3 & 37.2 & 42.3 \\
\hline (in millions of U.S. dollars) & 1,004 & 1,180 & 1,520 & 1,733 & 1,959 & 2,142 & 2,307 & 2,652 & 3,011 \\
\hline \multirow[t]{2}{*}{ (in pereent of tolal liquidity) } & 21.0 & 20.3 & 22.9 & 23.5 & 25.3 & 26.4 & 26.7 & 26.1 & 27.0 \\
\hline & \multicolumn{9}{|c|}{ (Annual percentage change) } \\
\hline Credit to the economy & 26.9 & 20.1 & 22.6 & 16.4 & 17,8 & 18.6 & 14.9 & 19.2 & 25.1 \\
\hline Claims on exterprises & 17.7 & 11.3 & 15.6 & 22.9 & 23.0 & 19.2 & 7.1 & 9.7 & 14.4 \\
\hline Claims on other sectors & 41.4 & 31.7 & 30.5 & 10.0 & 126 & 17.9 & 23.4 & 29.8 & 36.7 \\
\hline Broad money (M2) & 22,6 & 22.7 & 26.1 & 25.6 & 28.0 & 29.5 & 29.3 & 39.3 & 45.6 \\
\hline of which: total deposits & 37.6 & 25.3 & 34.3 & 33.6 & 37,9 & 37.8 & 37.1 & 34,0 & 40.3 \\
\hline Dong liquidity & 24.4 & 23.7 & 22.0 & 24.6 & 24.8 & 26.3 & 28.7 & 34.6 & 42.3 \\
\hline Currency outside banks & 2.9 & 18.1 & 10.9 & 7.4 & 4.8 & 7.6 & 7.9 & 54.1 & 61.8 \\
\hline Deposits & 51.3 & 28.5 & 30.8 & 36.0 & 37.8 & 37.0 & 40.5 & 24.4 & 32.7 \\
\hline \multirow[t]{2}{*}{ Foreign currency deposits } & 16.1 & 19.0 & 42.0 & 28.8 & 38.2 & 39.1 & 31.0 & 54.5 & 55.5 \\
\hline & \multicolumn{9}{|c|}{ (Change in percent of beginning of year broad money) } \\
\hline Nel foreign assets & 9.1 & 6.5 & 10.4 & 12.5 & 16.8 & 20.6 & 23.6 & 29.7 & 33.4 \\
\hline Net domestic assets & 13.5 & 16.3 & 15.7 & 13.1 & 11.1 & 8.9 & 5.7 & 9.6 & 12.2 \\
\hline Net claims on governuent & 0.3 & 4.5 & 0.0 & 4.9 & 3.6 & 1.2 & 0.1 & -5.3 & -7.9 \\
\hline Credit to the ecomomy & 20.9 & 16.2 & 17.8 & 126 & 13.6 & 14.0 & 11.0 & 13.6 & 17.7 \\
\hline Velooity $3 /$ & 4.3 & $4-2$ & 3.8 & 3.5 & 3.4 & 3.4 & 3.2 & 2.8 & 2.6 \\
\hline Money multiplier $\mathbf{4}$ / & 20 & 20 & 23 & 26 & 2.7 & 2.9 & 2.8 & 3.3 & 2.7 \\
\hline Currency to dong deposits (in percent) & 85.3 & 78.4 & 66.5 & 52.5 & 49.2 & 44.9 & 43.7 & 65.0 & 60.0 \\
\hline Currency to total deposits (in percent) & 57.2 & 53.9 & 44.5 & 35.7 & 32.7 & 29.5 & 28.7 & 41.1 & 37.7 \\
\hline
\end{tabular}

Sources: State Bank of Vietnam; and staff eutinakes.

1/ Comprising four state-owned comnercial banks and 24 nonstate-owned banks.

$2 /$ Includes D 2.4 trillion recapitalization of state-owned commercisl banks (SOCBs) in October 1998, under which frozen touns of SOCBs were written off on behalf of the govemnent and swapped for govemment equity in \$OCBs.

3/ Velocity is measured as the ratio of GDP to end-of-period broad money (M2).

4/ Money multiplier is measured as the ratio of braad money (M2) to reserve maney. 
Table 18. Vietham; Balance Sheet of the State Bank of Vietnam, 1995-2000

\begin{tabular}{|c|c|c|c|c|c|c|c|c|c|}
\hline & \multirow[t]{2}{*}{1995} & \multirow[t]{2}{*}{1996} & \multirow[t]{2}{*}{1997} & \multirow[t]{2}{*}{1998} & \multicolumn{4}{|c|}{1999} & \multirow{2}{*}{$\begin{array}{c}2000 \\
\text { March }\end{array}$} \\
\hline & & & & & March & June & Sept. & Dec. & \\
\hline & \multicolumn{9}{|c|}{ (in trillions of dong, end of pertiod) } \\
\hline Nex foreign assats & 11.0 & 14.0 & 19.4 & 22.1 & 24.1 & 27.7 & 33.9 & 40.8 & 44.7 \\
\hline Foreign assets & 15.2 & 20.0 & 25.6 & 29.1 & $3 \mathbf{L 2}$ & 34.7 & 41.1 & 48.0 & 51.8 \\
\hline Foneign liabilitios & -4.2 & -6.0 & -5.2 & -7.0 & -7.1 & -7.0 & -7.3 & -7.2 & -7.1 \\
\hline Net domestic essets & 15.8 & 18.2 & 16.4 & $\mathbf{1 6 . 6}$ & 15.1 & 10.9 & 9.4 & 17.4 & 13.6 \\
\hline Net domestic credit & 9.9 & 13.0 & 11.0 & 12.8 & 11.8 & 7.4 & 6.1 & 10.5 & 5.3 \\
\hline Net clainus on goventument & 3.0 & 5.2 & 40 & 6.3 & 5.0 & 0.3 & -1.1 & 0.2 & -5.9 \\
\hline Claints on banks & 6.8 & 7.7 & 6.8 & 6.5 & 6.7 & 7.1 & 7.2 & 10.3 & 11.1 \\
\hline Claims on enterprises and nonbank financial institutions & 0.1 & 0,1 & 0.2 & 0.0 & 0.0 & 0.0 & 0.0 & 0.0 & 0.0 \\
\hline Other itoms, pet. & 6.0 & 5.2 & 5.4 & 3.7 & 3.3 & 3.5 & 3.3 & 6.9 & 8.3 \\
\hline Reserve money & 26.8 & 32.2 & 35.8 & 38.7 & 39.2 & 38.6 & 43.3 & 58.2 & $\$ \$ .3$ \\
\hline Curnency in circulation & 20.0 & 23.8 & 26.3 & 28.3 & 28.2 & 27.4 & 28.7 & 44.9 & 44.8 \\
\hline Bank deposits & 6.3 & 7.8 & 9.3 & 10.4 & 11.0 & 11.2 & 14.6 & 13.3 & 13.5 \\
\hline Vault cash & 0.8 & 1.2 & 1.2 & 1.3 & 1.7 & 1.7 & 1.7 & 1.8 & 1.8 \\
\hline Required reserves 1 & 3.2 & 4.0 & 5.4 & 7.2 & 5.4 & 4.1 & 4.5 & 4.6 & 4.6 \\
\hline Exess reserves $1 /$ & 3.6 & 4.2 & 2.9 & 1.4 & 4.4 & 5.7 & 9.1 & 8.4 & 8.4 \\
\hline \multirow[t]{2}{*}{ Other deposits } & 0.5 & 0.6 & 0.2 & 0.0 & 0.0 & 0.0 & 0.0 & 0.0 & 0.0 \\
\hline & \multicolumn{9}{|c|}{ (Annual percantage change) } \\
\hline Net foreigh atsets & 67.7 & 27.6 & 38.1 & 14.1 & 25,9 & 39.6 & 53.1 & 69.1 & 85.4 \\
\hline Not demestic assets & -8.5 & 14.8 & -9.8 & 1.0 & -8.9 & -27.2 & -43.2 & 15.6 & -9.8 \\
\hline Net domestic credit & -16.2 & 31.8 & -13.6 & 17.1 & 9.8 & -225 & -52.4 & -10.6 & -55.3 \\
\hline Net claims on governuent & -24.7 & 72.1 & -22.3 & 56.6 & 16.4 & $-\$ 1.3$ & -116.9 & $-96,0$ & -216.5 \\
\hline Claims on banks & -12.6 & 13.5 & -11.9 & -3.8 & 7.0 & 20.6 & 10.1 & 53.6 & 65.9 \\
\hline Reserve money & 12.5 & 20.0 & 11.1 & 8.1 & 9.7 & 10.8 & 11.9 & 48.5 & 48.8 \\
\hline Currency in circulation & 3.2 & 18.9 & 10.3 & 7.6 & 4.7 & 7.7 & 1.5 & 59.4 & 59.1 \\
\hline \multirow[t]{2}{*}{ Bank deposits } & 43.3 & 23.8 & 19.2 & 11.6 & 26.6 & 19.6 & 40.2 & 20.6 & 22.5 \\
\hline & \multicolumn{9}{|c|}{ (Change in porsent of beginning of year nesve money) } \\
\hline Net foreign assets & 18.6 & 11.3 & 16.6 & 7.6 & 13.9 & 23.8 & 41.1 & 60.5 & 6.8 \\
\hline Net domestic assets & -6.1 & 8.7 & -5.5 & 0.5 & -4.1 & -15.8 & -20.0 & 2.4 & -6.6 \\
\hline Net domestic credit & -8.0 & 11.7 & -6.3 & 5.2 & 2.9 & -9.2 & -12.9 & -0.6 & -9.0 \\
\hline Of which: Net dxins on government & -4.2 & 8.1 & -3.6 & 6.4 & 2.0 & -11.2 & -15.1 & -11.6 & -10.4 \\
\hline \multicolumn{10}{|l|}{ Mernorandumn itern: } \\
\hline Gross official reserves (in millions of U.S. do.1]ans) $2 /$ & 1,323 & 1,673 & 1.857 & 1,769 & 1,903 & 1,989 & 2,246 & 2.711 & 2,816 \\
\hline (in weelss of next year's iniports) $3 /$ & 5.2 & 6.4 & 7.2 & 6.7 & 6.4 & 6.7 & 7.6 & 9.1 & 8.3 \\
\hline
\end{tabular}

Sources: State Bank of Vietnam; and staff eqtimates.

1) Staff estimate.

2/ Excludes forcign currency counterpart of govemenent foreign currency deposits at the State Bank of Vietnam.

$3 /$ Imports of goods and nonfactor services. 


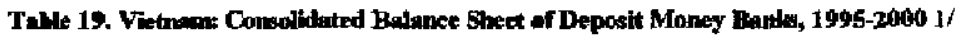

\begin{tabular}{|c|c|c|c|c|c|c|c|c|c|}
\hline & \multirow[t]{2}{*}{1995} & \multirow[t]{2}{*}{1996} & \multirow[t]{2}{*}{1997} & \multirow[t]{2}{*}{1998} & \multicolumn{4}{|c|}{1999} & \multirow{2}{*}{$\begin{array}{r}2000 \\
\text { March }\end{array}$} \\
\hline & & & & & March & June & Sept. & Dec. & \\
\hline & \multicolumn{9}{|c|}{ (In trillions of dong, end of period) } \\
\hline Net foreign assets & -0.1 & 0.2 & 1.6 & 9.1 & 12.5 & 15,0 & 15.9 & 20.8 & 27.9 \\
\hline Foreign ssets & 9.6 & 11.2 & 12.3 & 17.8 & 20.1 & 21.1 & 21.6 & 26.7 & 34.1 \\
\hline Foreign liabilities & -9.7 & -11.0 & -10.7 & -8.7 & -7.6 & $-6,2$ & -5.7 & -5.8 & -6.2 \\
\hline Net domestic & 33.2 & 41.2 & 54.7 & 66.4 & 68.6 & $T 22$ & 78.1 & 80.3 & 86,0 \\
\hline Net domestio credit from the commercial batiks & 43.8 & 50.0 & 62.6 & 74.7 & 77.7 & 822 & 84.3 & 89.4 & 99.1 \\
\hline Net oredit to government & 1.5 & -0.8 & 0.4 & 2.1 & 1.8 & 4.3 & 5.1 & 2.7 & 4.2 \\
\hline Credit to the ecowomy & 42.3 & 50.8 & 62.3 & 72.7 & 75.9 & 77.9 & 79.2 & 86.6 & 94.9 \\
\hline Stale enterprises & 24.1 & 26.8 & 31.0 & 38.1 & 39.6 & 39.8 & 38.4 & 41.8 & 43.3 \\
\hline Other sectors & 18.2 & 24.0 & 31.3 & 34.6 & 36.3 & 38.2 & 40.8 & 44.9 & 49.7 \\
\hline Of whtch: claims on nonbank finaspial institutions & 0.0 & 0.0 & 0.1 & 0.1 & 0.1 & 0.0 & 0.0 & 0.0 & 0.0 \\
\hline Banks' reserves & 7.6 & 9.3 & 9.5 & 9.9 & 11.4 & 11.6 & 15.3 & 15.6 & 14.3 \\
\hline Crecit from State Bank of Vietram & -5.8 & -6.1 & -5.6 & -4.2 & -4.4 & -5.0 & -6.4 & -10.6 & -11.1 \\
\hline Cepipital and reserves & -5.3 & 6.2 & -8.2 & -11.6 & -11.3 & -11.4 & -11.5 & -11.6 & -11.7 \\
\hline Dther iteme, net & -7.1 & -3.7 & -3.7 & -2.4 & -4.9 & -5.2 & -3.5 & -24 & -4.7 \\
\hline Depositts & 33.0 & 41.5 & 36.3 & 75.4 & 81.1 & 87.2 & 940 & 101.1 & 113.8 \\
\hline Dong deparits & 220 & 28.3 & 37.6 & 51.4 & 53.9 & 57.4 & 61.7 & 63.9 & 71.5 \\
\hline Densnd deposits & 6.9 & 10.2 & 14.7 & 20.2 & 19.1 & 20.1 & 22.8 & 25.6 & 27.1 \\
\hline Time deposits & 15.1 & 18.1 & 22.9 & 31.1 & 34,8 & 37.3 & 38.9 & 38.3 & $\mathbf{4 4 . 4}$ \\
\hline Foreign currency deposits & 12.1 & 13.2 & 18.7 & 24.1 & 27.2 & 29.9 & 32.3 & 37.2 & 42.3 \\
\hline Demand deposits & 8.9 & 11.0 & 15.6 & 21.2 & 24.2 & 27.2 & 29.5 & 34.6 & 40.0 \\
\hline \multirow[t]{2}{*}{ Import/restricted deposits } & 21 & 2.1 & 3.1 & 2.8 & 3.1 & 2.6 & 2.7 & 2.6 & 2.3 \\
\hline & \multicolumn{9}{|c|}{ (Annual pereentage change) } \\
\hline Credit to the exonomy & 26.8 & 20.0 & 22.6 & 16.7 & 18.0 & 18.6 & 14.9 & 19.2 & 25.1 \\
\hline State enterprises & 17.7 & 11.3 & I3.6 & 22.9 & 23.0 & 19.2 & 7.1 & 9.7 & 14.4 \\
\hline Other sectors & 41.3 & 31.5 & 30.4 & 10.6 & 13.0 & 17.9 & 23.4 & 29.8 & 36.7 \\
\hline Credil from Stale Banic of Vietram & 29.1 & 14.3 & 25.2 & 19.3 & 21.7 & 25.4 & 22.3 & 19.6 & 27.6 \\
\hline Total deposits & 35.8 & 25,4 & 35.7 & 34.1 & 38.2 & 37.8 & 37.1 & 34.0 & 40.3 \\
\hline Dong deposits & 48.6 & 28.7 & 32.8 & 36.7 & 38.1 & 37.1 & 40.6 & 24.4 & 32.7 \\
\hline Demand deposits & 37.3 & 48.6 & 43.8 & 37.9 & 30.3 & 40.7 & 45.0 & 26.5 & 41.7 \\
\hline Tüne deposits & 54.3 & 19.6 & 26.6 & 36.0 & 42.5 & 35.2 & 38.1 & 23.0 & 27.7 \\
\hline Foreign ourrency deposits & 16.1 & 19,0 & 42.0 & 28.8 & 38.2 & 39.1 & 31.0 & 34.5 & 55.5 \\
\hline Demand deposils & 20.8 & 23.7 & 41.4 & 36.1 & 43.4 & 44.5 & 33.3 & 62.7 & 65.6 \\
\hline Inaport/restrioled deposits & -0.1 & -0.9 & 45.5 & -8.1 & 7.4 & 0.8 & 10.2 & -6.8 & -23.7 \\
\hline \multicolumn{10}{|l|}{ Memorandum items: } \\
\hline Foreign ourrensy deposits (in millions of U.S. dollars) & 1,004 & 1,180 & 1,521 & 1,733 & 1,959 & 2,143 & 2,306 & 2,652 & 3,011 \\
\hline (annual percentage change) 2 & 16.4 & 17.5 & 28.8 & 14.0 & 29.0 & 29.7 & 30.2 & 53.0 & 53.7 \\
\hline (in peroent of total deposits) & 33.5 & 31.7 & 33.2 & 31.9 & 33.6 & 34.2 & 34.3 & 36.8 & 37.2 \\
\hline (change in millions of U.S. dollars) 3 / & 142 & 176 & 340 & 213 & 223 & 409 & 572 & 918 & 360 \\
\hline of which: demand deposits & 142 & 178 & 2801 & 223 & 208 & 423 & 579 & 932 & 380 \\
\hline
\end{tabular}

Soureas: Stato Bank of Vietnam; and staff estimnates.

1/ Comprising four state-owned and 24 nonstate-owned commercial banks.

2 Twelve-moith percentage changes.

3 Change since the begiming of the year. 
Table 20. Vietnam: Distribution of Credit, 1995-99

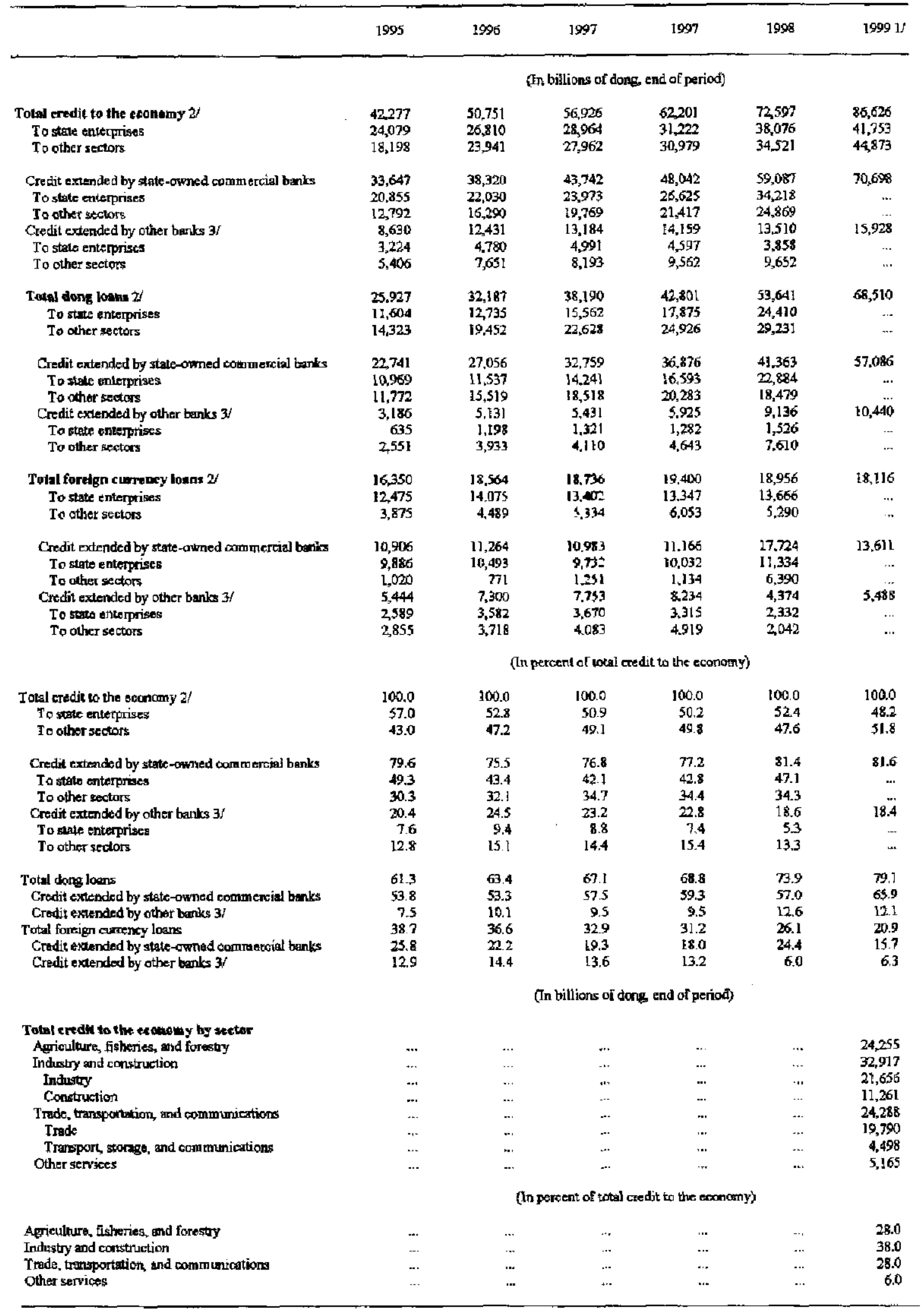

Soutrce: State Bank of Vietram; and staff cstirmiles.

I/ Beginning in 1999, credit to the economy by sector of ownership are estim ated rather than actual dais. 3 Excludes net credit to the govenment.

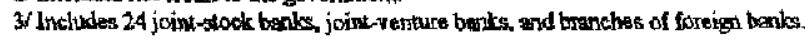


Table 21. Vietnam: Overdue Loans of Deposit Money Banks, 1995-2000 1/2/

\begin{tabular}{|c|c|c|c|c|c|c|c|c|c|}
\hline & \multirow[t]{2}{*}{1995} & \multirow[t]{2}{*}{1996} & \multirow[t]{2}{*}{1997} & \multirow[t]{2}{*}{1998} & \multicolumn{4}{|c|}{1999} & \multirow{2}{*}{$\begin{array}{c}2000 \\
\text { March }\end{array}$} \\
\hline & & & & & March & June & Sept. & $\overline{\text { Dec. }}$ & \\
\hline & \multicolumn{9}{|c|}{ (In billions of dong, end of period) } \\
\hline Deposit money banks & 3,337 & 4,726 & 7,682 & 8,697 & 9,078 & 10,374 & 11,333 & 11,470 & 12,479 \\
\hline State-owned commercial banks & 3,052 & 4,209 & 5,769 & 6,471 & 6,616 & 6,993 & 7,810 & 7,813 & 8,827 \\
\hline \multirow[t]{2}{*}{ Nonstate-owned banks } & 285 & 517 & 1,914 & 2,227 & 2,462 & 3,381 & 3,523 & 3,657 & 3,652 \\
\hline & \multicolumn{9}{|c|}{ (In percent of total loans) } \\
\hline Deposit money banks & 7.9 & 9.3 & 12.4 & 12.0 & 12.0 & 13.3 & 14.3 & 13.2 & 13.1 \\
\hline State-owned commercial banks & 9.1 & 11.0 & 12.0 & 11.0 & 10.6 & 11.0 & 12.1 & 11.1 & 11.0 \\
\hline \multirow[t]{2}{*}{ Nonstate-owned banks } & 3.3 & 4.2 & 13.5 & 16.4 & 18.0 & 23.3 & 23.7 & 23.0 & 24.4 \\
\hline & \multicolumn{9}{|c|}{ (In percent of total bank assets) } \\
\hline Deposit money banks & 4.8 & 5.5 & 7.4 & 6.8 & 6.7 & 7.2 & 7.4 & 7.2 & 7.1 \\
\hline State-owned commercial banks & 5.2 & 6.4 & 7.1 & 6.2 & 5.9 & 5.8 & 6.0 & 5.8 & 5.9 \\
\hline \multirow[t]{2}{*}{ Nonstate-owned banks } & 2.9 & 2.5 & 8.5 & 9.8 & 10.6 & 14.6 & 15.2 & 15.1 & 14.1 \\
\hline & \multicolumn{9}{|c|}{ (In percent of total overdues) } \\
\hline State-owned enterprises & 49.7 & 44.2 & 34.8 & 34.7 & 35.2 & $\ldots$ & $\ldots$ & $\ldots$ & $\ldots$ \\
\hline Cooperatives & 2.1 & 1.7 & 1.3 & 1.0 & 0.9 & ... & $\cdots$ & $\ldots$ & $\cdots$ \\
\hline Joint-stock companies & 5.3 & 11.8 & 45.7 & 46.7 & 46.4 & $\ldots$ & $\cdots$ & $\ldots$ & $\cdots$ \\
\hline Joint ventures & 0.2 & 0.6 & 0.6 & 1.1 & 0.7 & $\ldots$ & $\ldots$ & $\cdots$ & $\ldots$ \\
\hline \multirow[t]{2}{*}{ Private seotor } & 42.7 & 41.7 & 17.5 & 16.6 & 16.8 & $\ldots$ & $\ldots$ & $\cdots$ & $\cdots$ \\
\hline & \multicolumn{9}{|c|}{ (In percent of total loans to the sector) } \\
\hline State-owned enterprises & 6.9 & 7.8 & 8.6 & 7.9 & 8.1 & $\ldots$ & $\ldots$ & $\ldots$ & $\ldots$ \\
\hline Cooperatives & 26.3 & 29.7 & 40.2 & 35.1 & 33.6 & $\ldots$ & $\ldots$ & $\ldots$ & $\ldots$ \\
\hline Joint-stock companies & 3.7 & 7.4 & 34.3 & 36.7 & 360 & $\ldots$ & $\ldots$ & $\ldots$ & $\ldots$ \\
\hline Joint ventures & 0.4 & 1.0 & 1.2 & 2.0 & 1.5 & $\ldots$ & & $\ldots$ & $\ldots$ \\
\hline Private sector & 12.7 & 14.9 & 8.1 & 7.7 & 7.7 & $\ldots$ & $\ldots$ & $\ldots$ & $\ldots$ \\
\hline
\end{tabular}

Sources: State Bank of Vietnam; and staff estimates.

]/Based on nonstandand classification of nonperforming loans of four state-owned commercial banks and 24 nonstate-owned banks, which tends to underestimate the actual amount of bad loans.

2/Beginning June 1999, data on credit to the economy (including overdues) by sector of ownership not compiled by the State Bank of Vietnam in consolidated balance sheet of deposit money banks. 
Table 22. Vietnam: Nominal and Real Interest Rates, 1995-2000

(In percent per year, end of period)

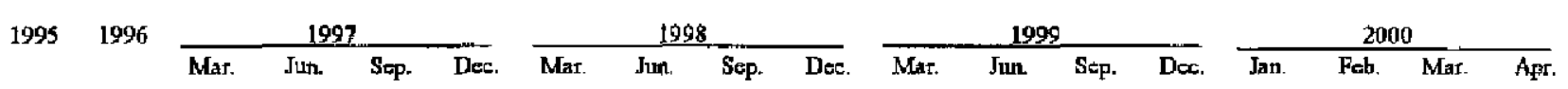

\section{Nombal mterest rates}

\section{Deposit rates 1}

Demand deposita

(h), houkeholds

Foreign currency demand deposits

Foreign currency deposits (uix-motsth)

\begin{tabular}{|c|c|c|c|c|c|c|c|c|c|c|c|c|c|c|c|c|c|}
\hline 8.7 & 6.2 & 6.5 & 6.2 & 4.6 & 4.6 & 5.4 & 5.5 & 5.5 & 5.5 & $\$ .1$ & 5.2 & 3.0 & 1.7 & 1.7 & 1.8 & 1.7 & 1.7 \\
\hline 18.2 & 9.1 & 19.4 & 9.9 & 7.8 & 8.1 & 9.4 & 9,9 & 9.7 & 9.7 & 9.4 & 9.1 & 5.1 & 4.0 & 3.7 & 3.7 & 3.7 & 3.6 \\
\hline$\ldots$ & $\ldots$ & $\ldots$ & $\ldots$ & $\ldots$ &.. & 2.7 & 2.7 & 2.4 & 2.4 & 2.6 & 2.3 & 2.3 & 23 & 1.6 & 1.6 & 1.5 & 1.5 \\
\hline$\ldots$ & $\cdots$ & $\cdots$ & $\ldots$ & $\ldots$ & $\ldots$ & 5.1 & 5.1 & 5.1 & 4.9 & 4.9 & 4.7 & 4.8 & 4.5 & 3.7 & 3.8 & 3.8 & 4.0 \\
\hline 28.3 & 15.9 & 15.4 & 15.2 & 12.7 & $12 . T$ & 15.2 & 15.0 & 14.7 & 14.7 & 13.9 & 13.7 & 12.7 & 11.7 & 11.4 & 11.4 & 10.8 & 10.2 \\
\hline 22.4 & 17.3 & 16.9 & 16.9 & 14.0 & 14.0 & 15.7 & 15.9 & 15.4 & 15.2 & $\$ 4.7$ & 14.0 & 12.7 & 11.7 & 11.7 & 11.7 & 11.4 & 10.5 \\
\hline 9.5 & 9.5 & 9.5 & 9.5 & 8.5 & 8.5 & B, S & 8.5 & 7.5 & 7.5 & 7.1 & 7.0 & 7.2 & 6.5 & 7.1 & 7.2 & 7.0 & 6.9 \\
\hline
\end{tabular}

Lending rates $2 /$

Working espital (short term)

Fixed capital (nedium term)

Foneign currency loars

Real interest rates $3 /$

Depesit rates

Demand deposits, enterprises

Savings deposits (three-monlh), horseholds

Foreign curnency demand deposits

Foreign cumency deposits (six-month)

\section{Lending rates}

Working capital (short term)

Fixed capital (medium term)

Forvign currency loang

\begin{tabular}{|c|c|c|c|c|c|c|c|c|c|c|c|c|c|c|c|c|c|}
\hline$\ldots$ & 24 & 3.0 & 4.4 & 1.0 & 0.8 & 8.6. & -2.6 & -3.0 & -3.5 & -3.5 & 0.1 & 0.1 & 1.6 & 2.1 & 2.9 & 3.5 & 3.8 \\
\hline 3.5 & 5.2 & 6.7 & 8.0 & 4,1 & 4.1 & 4.4 & 1.4 & 0.8 & 0.4 & 0.5 & 3.8 & 2.0 & 3.9 & 4.1 & 4.8 & 5.5 & 5.7 \\
\hline$\ldots$ & $\ldots$ & $\ldots$ & $\ldots$ & $\ldots$ & $\ldots$ & 16.7 & 5.7 & 11.8 & 3.6 & -6.1 & -2.1 & 0.7 & 3.8 & 3.4 & 4.1 & 4.5 & 4.6 \\
\hline .' & $m$ & ... & $\ldots$ & $\cdots$ & $\ldots$ & 19.3 & 8.2 & 14.5 & 6.0 & $-4,1$ & 0.1 & 2.9 & 5.2 & 5.6 & 6.3 & 7.0 & 3,0 \\
\hline 12.4 & $1 \pm .8$ & 11.6 & 13.3 & 8.9 & 8.6 & 10.0 & 6.2 & 5.4 & 4.9 & 4.6 & 8.2 & 9.4 & 11.7 & 11.8 & 12.6 & 12.7 & 12.4 \\
\hline 7.3 & โ3.2 & 13.1 & 15.0 & 10.2 & 9.9 & 10.5 & 7.0 & 6.0 & 5.4 & 5.3 & 8.4 & 9.4 & 11.7 & $12: 1$ & 12.9 & 13.4 & 12.8 \\
\hline-4.9 & 8.1 & 14.2 & 22.2 & 6.0 & 19,8 & 23.3 & 11.7 & 17.3 & 8.7 & -2.0 & 2.4 & 5.3 & 8.1 & 9,0 & 9.8 & 10.2 & 10.2 \\
\hline
\end{tabular}

\section{Mewarandem items}

Interest cate spread 4

Inflation 5/

Exchange rale (+ depreciation) of

\begin{tabular}{|c|c|c|c|c|c|c|c|c|c|c|c|c|c|c|c|c|c|}
\hline 10.2 & 6.8 & 5.0 & 5.3 & 4.9 & 4.6 & 5.8 & 5.2 & 5.0 & 5.0 & 4.5 & 4.6 & 7.6 & 7.7 & 7.7 & 7.7 & 7.1 & 6.6 \\
\hline 14.1 & 3.7 & 3.4 & 1.7 & 3.5 & 3.8 & 4.7 & 8.3 & 8.8 & 9.3 & 8.9 & 5.1 & 3.0 & 0.0 & -0.4 & -1.1 & -1.7 & -2.0 \\
\hline-0.9 & 2.4 & 7.8 & 13.5 & 1.1 & 14.6 & 19.0 & 11.5 & 18.8 & 10.6 & $=0.3$ & 0.6 & 1.4 & 1.5 & 1.4 & 1.4 & 1.1 & 1.0 \\
\hline
\end{tabular}

Sources: State Bank of Vietnan; and staff entimatos.

1/ Mandated deposit rates throught 1995; average of four state-owned commercial banks therentur.

$2 \prime$ Maxinum lending futen through 1595; averago of four state-owned commorcial banlss thereafter.

3/ Measured with respect to norninal interest rates at the end of each quarter and average mondhily inflation during that quarter usitig the exact Fisher formula. Real interest rates on foreigh currency deposits and loa exchatige rale depreciation using the same formula.

4/ Difference between interest rates on working capital and houscholds' throe-month sevings deposits.

5/ Average monthly inflation during preceding quarter, not seasonally adjusted.

6/ Average depreciation during preceding quarter, annualized. 
Table 23. Vietnam: Exchange Rate Developments, 1995-2000

\begin{tabular}{|c|c|c|c|c|c|c|}
\hline & 1995 & 1996 & 1997 & 1998 & 1999 & 2000 \\
\hline & \multicolumn{6}{|c|}{ (Twelve-month average; $1990=100$ ) } \\
\hline \multicolumn{7}{|l|}{ Effective exchange rates $1 /$} \\
\hline Nominal & 42.2 & 43.9 & 45.1 & 46.7 & 42.3 & $38.41 /$ \\
\hline \multirow[t]{2}{*}{ Real } & 122.9 & 131.1 & 135.4 & 146.5 & 137.4 & $134.9 \mathrm{l}$ \\
\hline & \multicolumn{6}{|c|}{ (Annual percentage change; - depreciation) } \\
\hline Nominal & -5.6 & 4.0 & 2.8 & 3.7 & -9.5 & -6.211 \\
\hline \multirow[t]{2}{*}{ Real } & 6.5 & 6,6 & 3.3 & 8.2 & -6.2 & $-5.81 /$ \\
\hline & \multicolumn{6}{|c|}{ (In units indicated) } \\
\hline Dong per U.S. dollar, end of period & 11,015 & 11,150 & 12,292 & 13,896 & 14,028 & $14,0852 /$ \\
\hline Dong per U.S. dollar, 12-month average & 11,038 & 11,033 & 11,706 & 13,297 & 13,944 & 14,026 \\
\hline Dong per SDR, end of period & 16,372 & 15,889 & 16,585 & 19,566 & 19,254 & 18,78621 \\
\hline \multirow[t]{2}{*}{ Dong per SDR, 12-month average } & 16,739 & 16,017 & 15,631 & 18,038 & 19,066 & $\cdots$ \\
\hline & \multicolumn{6}{|c|}{ (Annual percentage change, - depreciation) } \\
\hline Dong per U.S. dollar, end of period & 0.3 & -1.2 & -10.2 & -13.0 & -0.9 & -1.12 \\
\hline Dong per U.S. dollar, 12-month average & -0.6 & 0.0 & -6.1 & $-13,6$ & -4.9 & -1.5 \\
\hline Dong per SDR, end of period & -1.5 & 2.9 & -4.4 & -18.0 & 1.6 & -0.92 \\
\hline Dong per $S D R, 12$-month average & -6.7 & 4.3 & 2.4 & .15 .4 & -5.7 & $\ldots$ \\
\hline
\end{tabular}

Sources: Vietnamese authorities; and IMF, Information Notice System, and staff estimates.

1/ For 12-month period ending April 2000.

2/ For 12-month period ending June 2000. 
Table 24. Vietram: Bulance of Payments, 1995-1999

(In millions of U.S. dollars, unless otherwise indicated)

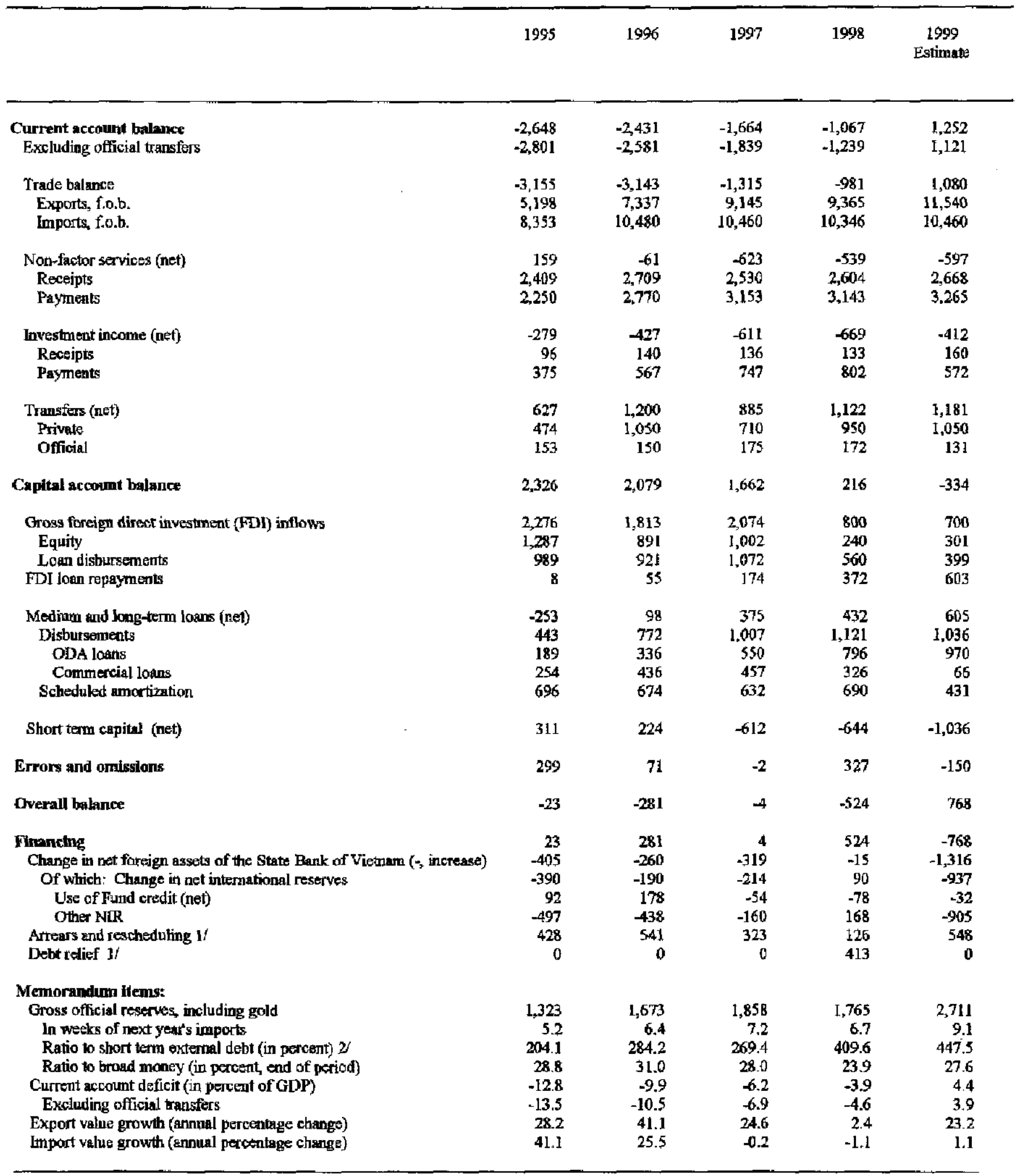

Sources: Data provided by the Viemamese authorities; and staff estimates and projections.

1/ London Chub rescheduling was concluded in early 1998.

2f Shori-term debt by remaining manurity refers to non-equity claims falling due over the next period. 
Tahle 25. Vietnam: Merchandise Erports by Commodity, 1995-99

\begin{tabular}{|c|c|c|c|c|c|}
\hline & 1995 & 1996 & 1997 & 1998 & $\begin{array}{r}1999 \\
\text { Estimate }\end{array}$ \\
\hline & \multicolumn{5}{|c|}{ (In millions of US. dollars, unless atherwise indicated) } \\
\hline Total exports, t. o.h & 5,198 & 7,337 & 9,145 & 9,365 & $11,5,40$ \\
\hline (ansulal percentage change) & 28.2 & 41.1 & 24.6 & 24 & 23.2 \\
\hline (Excluding oil) & 4,174 & 5,991 & 7,726 & 8,132 & 9,448 \\
\hline (amtulual pencentage change) & 30.9 & 43.5 & 29.0 & 5.3 & 16.2 \\
\hline Crude olis & 1.024 & 1,346 & 1,419 & 1,232 & 2092 \\
\hline Valume (von tirns) & 7,593 & 8,705 & 9,614 & 12,145 & 14,882 \\
\hline Unit value (USSiton) & 195 & 154 & 148 & 101 & 141 \\
\hline Conl & 90 & 155 & 111 & 102 & 96 \\
\hline Vohtme (1000 tots) & 2,620 & 3,647 & 3,449 & 3,162 & 3,260 \\
\hline Unit value (USS/ton) & 34 & 32 & 32 & 32 & 29 \\
\hline Rubber & 159 & 163 & 191 & 128 & 147 \\
\hline Volume (oou tons) & 132 & 122 & 195 & 191 & 265 \\
\hline Unit valpe (USS/ton) & 1,208 & 1,336 & 981 & 981 & 554 \\
\hline Rice & 496 & 855 & 870 & 1,024 & 1,025 \\
\hline Voluture (1000 tons) & 1,922 & 3,003 & 3,553 & 3,749 & 4,508 \\
\hline Unir valuo (USS/ton) & 258 & 285 & 245 & 273 & 227 \\
\hline ColTee & 565 & 337 & 491 & 594 & 585 \\
\hline Volume (000 tons) & 241 & 239 & 389 & 382 & 482 \\
\hline Unit valus (USS/ton) & 2,348 & 1,410 & 1,261 & 1,555 & 1,213 \\
\hline Marine products (incluating frozen items) & 431 & 651 & 781 & 818 & 951 \\
\hline Garments & 431 & 1,150 & 1,349 & 1,351 & 1,747 \\
\hline Footwear & 200 & 531 & 965 & 1,001 & 2,392 \\
\hline Handicrufts & 70 & 79 & 121 & 111 & 168 \\
\hline \multirow[t]{2}{*}{ Other } & 1,732 & 2,071 & 2,847 & 3,004 & 3,337 \\
\hline & \multicolumn{5}{|c|}{ (in percent of value of total exports) } \\
\hline Crode oil & 19.7 & 18.3 & 15.5 & 13.2 & 18.1 \\
\hline Conil & 1.7 & 2.1 & 1.2 & 1.1 & 0.8 \\
\hline Rubber & 3.1 & 2.2 & 2.1 & 1.4 & 1.3 \\
\hline Rice & 9.5 & 11.7 & 9.5 & 10.9 & 8.9 \\
\hline Coffee & 10,9 & 4.6 & $\$ .4$ & 6.3 & 5.1 \\
\hline Marine products & B.3 & B.9 & 8.5 & 8.7 & 8.2 \\
\hline Garnintents & 8.3 & 15.7 & 14.8 & 14.4 & 15.1 \\
\hline Footwear & 3.8 & 7.2 & 10.6 & 10.7 & 12.1 \\
\hline Handicmats & 1.3 & $\mathbf{1 . 1}$ & 1.3 & 1.2 & 1.5 \\
\hline \multirow[t]{2}{*}{ Other } & 33.3 & 28.2 & 31.1 & 32.1 & 28.9 \\
\hline & \multicolumn{5}{|c|}{ (It percentage points of growth in the value of expotts) } \\
\hline Crude oil & 3.9 & 6.2 & 1.0 & -2.0 & 9,2 \\
\hline Cosl & 0.4 & 1.2 & -0.6 & -0.1 & -0.1 \\
\hline Fubber & 0.6 & 0.1 & 0.4 & -0.7 & 0.2 \\
\hline Rice & 1.7 & 6.9 & 0.2 & 1.7 & 0.0 \\
\hline Coffee & 5.8 & -4.4 & 2.1 & 1.1 & -0.1 \\
\hline Matine products & -3.0 & 4.2 & 1.8 & 0.4 & 1.4 \\
\hline Garmetits & -1.1 & 13.8 & 2.7 & 0.0 & 4.2 \\
\hline Footwear & 1.9 & 6.4 & $\$ .9$ & 0.4 & 4.2 \\
\hline Handicrafts & 1.7 & 0.2 & 0.6 & -0.1 & 0.6 \\
\hline Other & 16.1 & 6.5 & 10.5 & 1.7 & 3.6 \\
\hline
\end{tabular}

Sourses: Ministry of Trade and Customs Office; and staff estimates. 
Table 26. Vhetnan: Merchandise Imports by Commodity, 1995-99

\begin{tabular}{|c|c|c|c|c|c|}
\hline & 1995 & 1996 & 1997 & 1998 & $\begin{array}{c}1999 \\
\text { Estimate }\end{array}$ \\
\hline & \multicolumn{5}{|c|}{ (In millions of U.S. dollars, unloss othenwize indicated) } \\
\hline Fontal ineparts, c.i.f. & 8,381 & 11,644 & 11.592 & 11,527 & 11,623 \\
\hline (ansural percentage change) & 43.8 & 38.9 & -0.5 & -0.6 & 0.8 \\
\hline Petrakeum products (gasoline, diesel, ete.) & 724 & 1,079 & 1,084 & 827 & 1,054 \\
\hline Volume ('000 tons) & 4,391 & 5,8003 & 5.947 & 6,830 & 7.403 \\
\hline Unit valua (USS/ton) & 165 & 186 & 184 & 121 & 142 \\
\hline Fertilizer & 554 & 628 & $\$ 25$ & 475 & 464 \\
\hline Volutes (C00 tons) & 2,508 & 2,832 & $2 ; 458$ & 3,454 & 3,782 \\
\hline Urnit valus (Us:/ton) & 221 & 222 & 173 & 137 & 123 \\
\hline Inesticite & 66 & 89 & 114 & 326 & 133 \\
\hline Volutine $(000$ tons) & 30 & 60 & 22 & 33 & 34 \\
\hline Unit velue (USSton) & 2,213 & 1,484 & 5,168 & 3,793 & 3,880 \\
\hline Stated and iron & 389 & 529 & 484 & 524 & 587 \\
\hline Volume (Wos0 toms) & 1.126 & 1,549 & 1,320 & 1,735 & 2,264 \\
\hline Lrit value (US\$/ton) & 345 & 342 & 367 & 302 & 259 \\
\hline Cement & 84 & 81 & 52 & 2 & 1 \\
\hline Volume (COO tons) & 1,459 & 1,302 & 878 & so & 10 \\
\hline Unit value (USt/ton) & 57 & 62 & 59 & 42 & $\$ 1$ \\
\hline Motorcydes & 423 & 434 & 243 & 351 & 399 \\
\hline Volume (000 unit) & 404 & 472 & 220 & 384 & 414 \\
\hline Unit value (USS/luit) & 1,048 & 919 & 1,104 & 915 & 964 \\
\hline Mowr cora and truckss & 148 & 155 & 136 & 130 & 89 \\
\hline Volurne (voxi units) & 24.5 & 191 & 14.0 & 17.2 & 10.7 \\
\hline Unit value (USSrunit) & 6.054 & 8,131 & 9,221 & 7,575 & 8,263 \\
\hline Wheat floor & 65 & $\infty 0$ & 48 & 62 & 29 \\
\hline Volume (co00 tons) & 233 & 296 & 167 & $2 n$ & 143 \\
\hline Unit malue (USsiton) & 279 & 303 & 237 & 227 & 200 \\
\hline Textilt yorn (fibers) & 134 & 158 & 159 & 175 & 154 \\
\hline Volume ( 4000 tons) & 52 & 74 & 77 & 130 & 160 \\
\hline Un'x value (USStion) & 2,592 & 2,128 & 2,070 & 1,350 & 3.216 \\
\hline Cottan & 91 & 66 & 110 & 92 & 91 \\
\hline Laxther and partheat moturial & 305 & 531 & 897 & 821 & 1,096 \\
\hline Cigarette material (including tobacco) & 83 & 78 & 83 & 111 & 88 \\
\hline Machinery and equlpmost (inchuding aircreft) $2 /$ & 2,097 & 3,800 & 1,777 & 2,052 & 2,005 \\
\hline \multirow[t]{2}{*}{ Other } & 3,219 & 3,926 & 5,972 & 5,779 & 5,394 \\
\hline & \multicolumn{5}{|c|}{ In pereant of value of total inports) } \\
\hline Petroleurn produets & 8.6 & 93 & 9.4 & 7.2 & 9.1 \\
\hline Fartilizer & 6.6 & 5.4 & 3.7 & 4.1 & 4.0 \\
\hline Inserticide & 0.8 & 0.8 & 1.0 & 1.1 & 1.1 \\
\hline Steel and iron & 4.6 & 4.5 & 4.2 & 4.5 & 5.0 \\
\hline Cemant & 1.0 & 0.7 & 0.4 & 0.0 & 0.0 \\
\hline Motsoroyeles & 5.0 & 3.7 & 21 & 3.0 & 3.4 \\
\hline Motor and trucks & 1.8 & 1.3 & 1.2 & 1.1 & 0.8 \\
\hline Wheat Alour & 0.8 & 0.8 & 0.4 & 0.5 & 0.2 \\
\hline Textile yarm & 1.6 & I. 4 & 1.4 & 1.5 & 3.7 \\
\hline Cotton & 1.1 & 0.6 & 0.9 & 0.8 & 0.8 \\
\hline Loether and garment material & 3.6 & 4.6 & 7.7 & 7.1 & 9.4 \\
\hline Cigartette material & 1.0 & 0.7 & 0.7 & 1.0 & 0.8 \\
\hline Machinery and equiprtont & 25.0 & 32.6 & 15.3 & 17.8 & 17.3 \\
\hline Other & 38.4 & 33.7 & 51.5 & 50.1 & 46.4 \\
\hline
\end{tabular}

Souross: Ministry of Trade and Cutoms Officc; and staff extinates. 
Toble 27. Vietnam; Direction of Trade, 1995-99 1/ (In percent of total exports of imports)

\begin{tabular}{|c|c|c|c|c|c|}
\hline & 1995 & 1996 & 1997 & 1998 & 1999 \\
\hline \multicolumn{6}{|l|}{ Exports } \\
\hline Industrial countries or regions & 66.0 & 68.4 & 67.2 & 73.1 & 73.4 \\
\hline Japan & 26.8 & 21.3 & 18.2 & 18.0 & 17.6 \\
\hline Hong Kong SAR & 4.7 & 4.3 & 4.7 & 2.4 & 1.8 \\
\hline Korea & 4.3 & 7.7 & 4.5 & 1.9 & 1.7 \\
\hline Singapore & 12.7 & 17.8 & 33.2 & 4.4 & 4.7 \\
\hline France & 3.1 & 2.0 & 2.6 & 5.8 & 5.1 \\
\hline Germany & 4.0 & 3.1 & 4.5 & 9.2 & 9.0 \\
\hline Italy & 1.0 & 0.7 & 1.3 & 2.8 & 2.7 \\
\hline Netheriands & 1.5 & 2.0 & 2.9 & 2.5 & 2.3 \\
\hline Switzerland & 1.1 & 2.1 & 3.6 & 0.6 & 0.6 \\
\hline United Kingdom & 1.4 & 1.7 & 2.9 & 4.2 & 4.4 \\
\hline Other Europe (industrialized) 2 & 0.9 & 1.4 & 2.2 & 8,1 & 7.8 \\
\hline United States & 3.1 & 2.8 & 3.2 & 62 & 5.8 \\
\hline Canada & 0.3 & 0.4 & 0.7 & 1.4 & 1.3 \\
\hline Australia & 1.0 & 0.9 & 2.5 & 5.4 & 8.4 \\
\hline New Zealand & 0.0 & 0.1 & 0.2 & 0.3 & 0,2 \\
\hline Developing countries & 34.0 & 31.6 & 32.8 & 26.9 & 26.6 \\
\hline Chins (mainland) & 6.6 & 4.7 & 3.2 & 2.2 & 3.0 \\
\hline Indoresix & 1.0 & 0.6 & 0.5 & 0.6 & 0.5 \\
\hline Malaysia & 4.3 & 7.7 & 4.5 & 1.9 & 1.7 \\
\hline Philippines & 0.8 & 1.8 & 2.6 & 4.2 & 3.8 \\
\hline Russia & 1.5 & 1.2 & 1.4 & 0.6 & 0.2 \\
\hline Taiwan POC & 8.1 & 7,4 & 8.9 & 3.5 & 3.2 \\
\hline Thailand & 1.9 & 1.5 & 2.6 & 24 & 2.2 \\
\hline Other & 9.9 & 6.7 & 7.1 & 11.4 & 12.0 \\
\hline \multicolumn{6}{|l|}{ Imports } \\
\hline Industrial countries or fegions & 61.5 & 67.8 & 66.7 & 58.1 & 58.4 \\
\hline Japan & 11.2 & 11.3 & 13.0 & 11.8 & $\mathbf{1 4 . 1}$ \\
\hline Hong Kong SAR & 5.1 & 7.1 & 5.2 & 4.5 & 3.9 \\
\hline Korea & 15.4 & 16.0 & 13.5 & 12.1 & 12.4 \\
\hline Singapore & 17.5 & 18.2 & 18.4 & 13.4 & 12.9 \\
\hline Frange & 3.4 & 3.7 & 4,8 & 2.7 & 2.7 \\
\hline Germany & 2.2 & 2.6 & 2.4 & 3.2 & 2.2 \\
\hline Italy & 0.7 & 1.0 & 0.9 & 1.0 & 1.0 \\
\hline Netherlands & 0.4 & 0.5 & 0.4 & 0.3 & 0.3 \\
\hline Switzerlaud & 0.9 & 1.3 & 1.2 & 0.5 & 0.5 \\
\hline United Kingdom & 0.6 & 0.8 & 0.9 & 1.0 & 1.1 \\
\hline Other Europe (industrialized) $2 /$ & 0.9 & 1.4 & 1.6 & 23 & 24 \\
\hline United States & 1.6 & 2.2 & 2.2 & 24 & 2.5 \\
\hline Canada & 0.3 & 0.3 & 0.3 & 0.3 & 0.3 \\
\hline Ansiralia & 1.2 & 1.2 & 1.7 & 2.1 & 1.8 \\
\hline New Žealand & 0.0 & 0.2 & 0.2 & 0.4 & 0.4 \\
\hline Developing coutritries & 38.5 & 32.2 & 33.3 & 41.9 & 41.6 \\
\hline Ohint (mainland) & 4.0 & 3.0 & 3.5 & 9.1 & 8.6 \\
\hline Indonesia & 2.3 & 1.3 & 1.7 & 2.1 & 2.0 \\
\hline Malaygia & 2.3 & 1.8 & 2.0 & 3.3 & 3.3 \\
\hline Philippines & 0.3 & 0.3 & 0.3 & 0.8 & 0.8 \\
\hline Rubsia & 1.8 & 1.7 & 1.4 & 2.4 & 1.4 \\
\hline Taiwan POC & 11.1 & 11.3 & 12.8 & 10.8 & 11.0 \\
\hline Thailand & 5.4 & 4.4 & 5.0 & 5.3 & 5.4 \\
\hline Other & 11.3 & 8.4 & 6.7 & 8.1 & 9.0 \\
\hline \multicolumn{6}{|l|}{$\begin{array}{l}\text { Mernorandum ilems: } \\
\text { European Union }\end{array}$} \\
\hline Exponty & 11.9 & 10.9 & 16.1 & 32.1 & 30.9 \\
\hline Imports & 8.1 & 9.9 & 11.0 & 10.4 & 9.6 \\
\hline \multicolumn{6}{|l|}{ ASEAN $3 /$} \\
\hline Exports & 20.6 & 29.4 & 23.8 & 13.9 & 13.2 \\
\hline Imports & 27.8 & 26.1 & 27.8 & 25.4 & 24.9 \\
\hline
\end{tabular}

Sounces: Vietnam Customg Office; IMF, Direction of Trade Statistics, and staff estimates.

1/ For 1998 and 1999, sane data reflect partser country data.

2/Defined as Austria, Belginm, Dentmatk, Fïland, Greece, lceland, Ireland, Lexembourg, Norway, Portugal, Spain, authd Sweden. 3/Including Lao PDR and Myaumar beginting in 1997 and Cambodia in 1999. 
Table 28. Vietnam: Commitments of Foreign Direct Investment, 1995-99 1/

\begin{tabular}{|c|c|c|c|c|c|}
\hline & 1995 & 1996 & 1997 & 1998 & 1999 \\
\hline & \multicolumn{5}{|c|}{ (In millions of U.S. dollars) } \\
\hline Industry & 2,477 & 2,712 & 1,815 & 842 & 805 \\
\hline Heavy industries & 1,342 & 1,347 & 746 & 553 & 369 \\
\hline Export processing zones & 0 & 94 & 208 & 0 & 0 \\
\hline Light industries & 668 & 613 & 508 & 208 & 240 \\
\hline Food & 466 & 658 & 353 & 81 & 196 \\
\hline Oil and gas & 32 & 80 & 107 & 1,358 & 46 \\
\hline Construction & 746 & 3,937 & 1,056 & 148 & 198 \\
\hline Transportation and communications & 386 & 748 & 962 & 312 & 137 \\
\hline Real estate & 2,666 & 917 & 384 & 899 & 148 \\
\hline Hotels and tourism & 1,395 & 399 & 188 & 784 & 148 \\
\hline Office property and apartments & 1,270 & 519 & 195 & 115 & 0 \\
\hline Agriculture, forestry, and fisheries & 158 & 119 & 133 & 88 & 59 \\
\hline Services & 144 & 128 & 197 & 250 & 168 \\
\hline \multirow[t]{2}{*}{ Total } & 6,608 & 8,640 & 4,654 & 3,897 & 1,563 \\
\hline & \multicolumn{5}{|c|}{ (In percent of total) } \\
\hline Industry & 37.5 & 31,4 & 39.0 & 21.6 & 51.5 \\
\hline Heavy industries & 20.3 & 15.6 & 16.0 & 14.2 & 23.6 \\
\hline Export processing zones & 0.0 & 1.1 & 4.5 & 0.0 & 0.0 \\
\hline Light industries & 10.1 & 7.1 & 10.9 & 5.3 & 15.4 \\
\hline Food & 7.1 & 7.6 & 7.6 & 2.1 & 12.5 \\
\hline Oil and gas & 0.5 & 0.9 & 2.3 & 34.9 & 3.0 \\
\hline Construction & 11.3 & 45.6 & 22.7 & 3.8 & 12.7 \\
\hline Transportation and communications & 5.8 & 8.7 & 20.7 & 8.0 & 8.8 \\
\hline Real estate & 40.3 & 10.6 & 8.2 & 23.1 & 9.5 \\
\hline Fotels and tourism & 21.1 & 4.6 & 4.0 & 20.1 & 9.5 \\
\hline Office property and apartments & 19.2 & 6.0 & 4.2 & 3.0 & 0.0 \\
\hline Agriculture, forestry, and fisheries & 2.4 & 1.4 & 2.9 & 2.2 & 3.8 \\
\hline Services & 2.2 & 1.5 & 4.2 & 6,4 & 10.8 \\
\hline Total & 100.0 & 100.0 & 100.0 & 100.0 & 100.0 \\
\hline
\end{tabular}

Sources: Ministry of Planning and Investment (MPI) and State Bank of Vietnam; and staff estimates.

1/ As reported by MPI. Inchudes investments by domestic joint venture partners. 
Table 29. Vietnam: Disbursements of Foreign Direct Investment, 1995-99 If

\begin{tabular}{|c|c|c|c|c|c|}
\hline & 1995 & 1996 & 1997 & 1998 & $\begin{array}{c}1999 \\
\text { Preliminary }\end{array}$ \\
\hline & \multicolumn{5}{|c|}{ (In millions of U.S. dollars) } \\
\hline Industry & 1,118 & 1,117 & 704 & 347 & 612 \\
\hline Heavy industries & 539 & 490 & 306 & 207 & 383 \\
\hline Export processing zones & 0 & 30 & 72 & 0 & 47 \\
\hline Light industries & 347 & 342 & 195 & 103 & 105 \\
\hline Food & 231 & 254 & 131 & 37 & 78 \\
\hline Qil and gas & 14 & 64 & 61 & 843 & 185 \\
\hline Construction & 264 & 1,138 & 381 & 52 & 115 \\
\hline Transportation and communications & 371 & 271 & 894 & 302 & 95 \\
\hline Real estate & 1,131 & 330 & 154 & 61 & 301 \\
\hline Hotels and tourism & 681 & 136 & 82 & 32 & 121 \\
\hline Office property and apartments & 450 & 194 & 72 & 29 & 180 \\
\hline Agriculture, forestry, and fisheries & 82 & 55 & 62 & 37 & 93 \\
\hline Services & 68 & 57 & 80 & 164 & 72 \\
\hline Total disbuysements & 3,048 & 3,032 & 2,336 & 1,806 & 1,474 \\
\hline \multirow[t]{2}{*}{ Total disbursements: staff estimates $2 /$} & 2,276 & 1,813 & 2,074 & 800 & 700 \\
\hline & \multicolumn{5}{|c|}{ (in percent of total) } \\
\hline Industry & 36.7 & 36.8 & 30.1 & 19.2 & 41.5 \\
\hline Heavy industries & 17.7 & 162 & 13.1 & 11.5 & 26.0 \\
\hline Export processing zones & 0.0 & 1.0 & 3.1 & 0.0 & 3.2 \\
\hline Light industries & 11.4 & 11.3 & 8.3 & 5.7 & 7.1 \\
\hline Food & 7.6 & 8.4 & 5.6 & 2.0 & 5.3 \\
\hline Oil and gas & 0.5 & 2.1 & 2.6 & 46.7 & 12.6 \\
\hline Construction & 8.7 & 37.5 & 16.3 & 2.9 & 7.8 \\
\hline Transportation and communications & 12.2 & 8.9 & 38.3 & 16.7 & 6.4 \\
\hline Real estate & 37.1 & 10.9 & 6.6 & 3.4 & 20.4 \\
\hline Hotels and tourism & 22.4 & 4.5 & 3.5 & 1.8 & 8.2 \\
\hline Office property and apartments & 14.8 & 6.4 & 3.1 & 1.6 & 12.2 \\
\hline Agriculture, forestry and fisheries & 2.7 & 1.8 & 2.6 & 2.0 & 63 \\
\hline Services & 2.2 & 1.9 & 3.4 & 9.1 & 4.9 \\
\hline Total & 100.0 & 100.0 & 100.0 & 100.0 & 100.0 \\
\hline \multicolumn{6}{|l|}{ Memorandum item: } \\
\hline Total disbursements (in percent of GDP) & 14.7 & 12.3 & 8.7 & 6.7 & 5.2 \\
\hline
\end{tabular}

Sources: Ministry of Planning and Investment (MPI); and staff estimates.

$1 /$ Data on disbursements classified by industry as reported by MPI. Calculated as total reported disbursements less equity contributions of domestic partners. Thus, data include domestic borrowing by the joint venture. Data are subject to extensive revision.

2/ Staff estimates are based on neporied foreign equity inflows plus fortign borrowings by joint ventures, as reported by the State Bank of Vietnam. For 1998 and 1999 , also based on other indicators of investrnent inflows. 
Table 30. Vietnam: Foreign Direct Investunent by Country of Origin, 1995-99 1/

\begin{tabular}{|c|c|c|c|c|c|}
\hline & \multicolumn{3}{|c|}{ Commitments 2 I } & \multicolumn{2}{|c|}{ Disbursements } \\
\hline & 1995 & 1996 & 1997 & 1998 & $\begin{array}{r}1999 \\
\text { Estimate }\end{array}$ \\
\hline & \multicolumn{5}{|c|}{ (In millions of U.S. dollars) } \\
\hline Singapore & 573 & 2,718 & 478 & 224 & 152 \\
\hline Taiwan POC & 1,060 & 485 & 222 & 194 & 116 \\
\hline Korea & 594 & 749 & 628 & 200 & 191 \\
\hline Japan & 1,183 & 591 & 643 & 541 & 415 \\
\hline British Virgin Islands & 461 & 1,290 & 191 & 128 & 18 \\
\hline Hong Kong SAR & 135 & 251 & 223 & 105 & 158 \\
\hline Malaysia & 98 & 84 & 151 & 111 & 95 \\
\hline Thailand & 138 & 238 & 237 & 42 & 10 \\
\hline United States & 503 & 81 & 172 & 11 & 41 \\
\hline France & 112 & 91 & 52 & 74 & 64 \\
\hline Australia & 112 & 44 & 3 & 6 & 62 \\
\hline Other & 723 & 618 & 1,182 & 169 & 149 \\
\hline \multirow[t]{2}{*}{ Total } & 5,692 & 7,240 & 4,183 & 1,806 & 1,474 \\
\hline & \multicolumn{5}{|c|}{ (In percent of total) } \\
\hline Singapore & 10.1 & 37.5 & 11.4 & 12.4 & 10.3 \\
\hline Taiwan POC & 18.6 & 6.7 & 5.3 & 10.7 & 7.9 \\
\hline Korea & 10.4 & 10.3 & 15.0 & 11,1 & 13.0 \\
\hline Japan & 20.8 & 8.2 & 15,4 & 30.0 & 28.2 \\
\hline British Virgin Islands & 8.1 & 17.8 & 4.6 & 7.1 & 1.2 \\
\hline Hong Kong SAR & 2.4 & 3.5 & 5.3 & 5.8 & 10.7 \\
\hline Malaysia & 1.7 & 1.2 & 3.6 & 6.2 & 6.5 \\
\hline Thailand & 2.4 & 3.3 & 5.7 & 2.3 & 0.7 \\
\hline United States & 8.8 & 1.1 & 4.1 & 0.6 & 2.8 \\
\hline France & 2.0 & 1.3 & 1.3 & 4.1 & 4.4 \\
\hline Australia & 2.0 & 0.6 & 0.1 & 0.4 & 4.2 \\
\hline Other & 12.7 & 8,5 & 28.3 & 9.3 & 10.1 \\
\hline Total & 100.0 & 100.0 & 100.0 & 1000 & 100.0 \\
\hline
\end{tabular}

Sources: Ministry of Planning and Investment (MPI); and staff estimates.

1/ Data as reported by MPI. See Table 29 for qualifications.

2/ Data on country distribution of FDI commitments not revised, as were data in Table 28. 
Table 31. Vietnam: Extermal Debt and Debt Service Obligations, 1995-99 1/

\begin{tabular}{|c|c|c|c|c|c|}
\hline & 1995 & 1996 & 1997 & 1998 & $\begin{array}{c}1999 \\
\text { Estimate }\end{array}$ \\
\hline \multirow[b]{2}{*}{ Total convertible currency debt stock } & \multicolumn{5}{|c|}{ (In millions of U.S. dollars, end of period) } \\
\hline & 7,259 & 9,029 & 10,336 & 10,319 & 10,577 \\
\hline Medium- and long-term debt stock & 6.478 & 8,024 & 9,551 & 9,645 & 10,020 \\
\hline Public sector & $15,12]$ & 15,308 & 16,028 & 15,939 & 16,598 \\
\hline Public sector convertible debt & 4,524 & 5,081 & 5,557 & 5,424 & 6,083 \\
\hline Official loans (excl. Russia and IMF) & 2,885 & 2,922 & 3,141 & 3,290 & 4,159 \\
\hline Commercial (London Club) & 907 & 978 & 923 & 666 & 666 \\
\hline State-owned enterprises 2 & 238 & 525 & 885 & 974 & 787 \\
\hline IMF & 377 & 538 & 490 & 377 & 354 \\
\hline Other & 59 & 59 & 59 & 59 & 59 \\
\hline Private sector & 1,953 & 2,943 & 3,994 & 4,221 & 3,937 \\
\hline FDI-related & 1,851 & 2,718 & 3,615 & 3,803 & 3,599 \\
\hline Other & 102 & 225 & 379 & 418 & 337 \\
\hline Shart-term deft stock & 781 & 1,005 & 785 & 674 & $5 \$ 7$ \\
\hline Total crternal debt senvice & 920 & 982 & 1,293 & 1,583 & 1,525 \\
\hline Principal & 704 & 684 & 860 & 1,139 & 1,062 \\
\hline Interest & 216 & 298 & 433 & 414 & 463 \\
\hline Public debt & 869 & 844 & 881 & 922 & 602 \\
\hline Principal & 692 & 621 & 660 & 708 & 363 \\
\hline Interest & 177 & 222 & 221 & 214 & 239 \\
\hline \multirow{4}{*}{$\begin{array}{c}\text { Private debt } \\
\text { Principal } \\
\text { ln }\end{array}$} & $\$ 2$ & 139 & 413 & 661 & 923 \\
\hline & 12 & 63 & 201 & 431 & 699 \\
\hline & 40 & 76 & 212 & 230 & 224 \\
\hline & \multicolumn{5}{|c|}{ (In percent of GDP) } \\
\hline \multirow{2}{*}{$\begin{array}{l}\text { Total debt service } \\
\text { of which; public }\end{array}$} & 4.4 & 4.0 & 4.8 & 5.8 & 5.4 \\
\hline & 4.2 & 3.4 & 3.3 & 3.4 & 2.1 \\
\hline \multirow{3}{*}{$\begin{array}{l}\text { Total convertible currency debt stock } \\
\text { of which: public }\end{array}$} & 35.0 & 36.6 & 38.6 & 38.1 & 37.1 \\
\hline & 31.2 & 20.6 & 20.7 & 20.0 & 21.4 \\
\hline & \multicolumn{5}{|c|}{ (In percent of exports of goods and nonfactor seavices) } \\
\hline \multirow{2}{*}{$\begin{array}{l}\text { Total deht service } \\
\text { of which: public }\end{array}$} & 12.1 & 9.8 & 11.1 & 13.2 & 10.7 \\
\hline & 11.4 & 8.4 & 7.5 & 7.7 & 4.2 \\
\hline \multirow{2}{*}{$\begin{array}{l}\text { Total convertible currency debł stock } \\
\text { of which: public }\end{array}$} & 85.2 & 79.9 & 81.8 & 86.2 & 70.5 \\
\hline & $\$ 9.5$ & 50.5 & 47.6 & 45.3 & 42.8 \\
\hline \multicolumn{6}{|l|}{ Mernorandum item: } \\
\hline Nonconvertible debt 3 / & 10,597 & 10,227 & 10.471 & 10,515 & 10.515 \\
\hline
\end{tabular}

Sources: Vietnamese authorities; and staff estimates.

1/ Comvertible currency debt only.

$2 /$ Includes loans to state-owned enterprises not classified elsewhere.

3/ In milions of transferable rubles. 


\section{Recent Developments in the External Trade System}

During the 1990s, Vietnam's trade system evolved from comprehensive control of the balance of payments towards protection for inport substituting industries. In the wake of the Asian crisis, trade restrictions were tightened in 1998 and early 1999 to protect the balance of payments and preferred domestic sectors. Some liberalizing steps bave been taken since late 1999 as concerns within the government grew over the link between high protection and the poor competitiveness of domestic industries. Many of these industries, often financed by domestic banks or through external borrowing, are now characterized by high production costs, excess capacity, and debt service difficulties. Moreover, as the liberalization under the ASEAN Free Trade Area (AFTA) is entering a more intensive phase, the potential impact of regional integration by 2006 on domestic industries is now becoming apparent. Thus, this note also includes a summary of Vietnam's commitments under AFTA.

\section{Foreign trading rights}

Decree 57 issued in July 1998 effectively extended the right to export unrestricted goods to all domestic enterprises. This permitted many more producers 10 deal directly with importing firms in Vietnam's export markets, and, as a result, to develop greater knowledge of market requirements and eliminate some inefficient intermediaries. Together with market opening agreements with the European Union and Japan, these reforms contributed to the substantial growth in manufactured exports in 1999. As a further step, the right for domestic enterprises to import any goods not subject to restrictions was included in the Enterprise Law (passed in June 1999), but the implementing regutations have not yet been issued.

Trading activities of foreign-invested enterprises are governed by the Foreign Investment Law (FIL). The authorities have stated that the right of foreign-invested enterprises to export is the same as for domestic enterprises. Regarding imports, foreign-invested enterprises can import inputs for production, but because they are not approved for trading activities (if they are set up for manufacturing), they cannot import other products in their line of business that may be needed to fill out their product range. Amendments passed in May 2000 to the Foreign Investment Law did not change this situation.

\section{Quantitative restrictions}

Effective April 1, 2000, quantitative restrictions (QRs) were removed from 8 of the 19 items subject to $Q R s$. The items liberalized were liquid soda, raw material for plastics, fertilizer, ceramic and glass consumer goods, bicycles, ceramic sanitary wares, consumer eiectric fans, and plastic packaging. Tariffs on these items were increased to 100 percent for some glass consumer products and 80 percent for bicycles, but the rate on fertilizer was only raised to 5 percent.

More generally, there appears to be a much greater easing of the import regime than implied by the removal of QRs. In the first half of 2000 , imports rose by an estimated 34 percent 
(over the same period in 1999), after three years of no increase in the value of total imports (despite large increases in oil prices, high export growth in 1999, and real GDP growth of 3-5 percent during these three years).

The goods remaining subject to $Q R$ s are ceramic and granite tiles, vegetable oil, window glass, paper (newsprint, writing, and packaging), construction steel, cement and clinker, motor cars for 16 or fewer passengers, motorcycles and kits, sugar, alcoholic beverages, and petroleum products. The last two items were removed from the list of items subject to import licensing (Decision 242/1999/QD-TTg), but are under separate controlling instruments that still restrict the total value of permitted imports.

\section{Tariffs}

In early 1999 , a new and substantially revised the tariff schedule was introduced. It reduced the maximum standard tariff rate to 50 percent; about 1 percent of line items had rates above this level, mainly alcoholic beverages and motor vehicles. This new schedule was also more in line with the harmonized standard nomenclature, increasing the total number of tariff items to about 6,000 .

In early 2000 , the tariff schedule was again revised, partly to reflect the tariff increases needed for the newly liberalized imports. These changes contributed to the increase in the number of tariff rates to 19 from 12 in 1999. Notwithstanding the increases in tariff rates on the liberalized items, the average tariff rate from the schedule remained at 15.5 percent, since the number of items with rates of 20 percent or less increased slightly.

\section{Bilateral trade agreements}

The bilateral trade agreement under discussion with the United States, would give Vietnam normal trade access to the U.S. market (subject to annual approval) in exchange for Vietnam phasing in steps to liberalize nontariff barriers, customs procedures, investment measures, and entry requirements for some services, and for reducing some tariffs. The framework was agreed in principle in June 1999, but formal approval by Vietnam was not given. In early 2000 , the Vietnamese authorities requested further discussions on some of the items in the agreement and discussions resumed in early July 2000. 
Vietnam: Summary of Normal Tariff Schedule, 1999-2000 ${ }^{\text {if }}$

Rates in 1999

\begin{tabular}{rrr}
\hline Bands & \multicolumn{2}{c}{ Number of lines: } \\
\cline { 2 - 3 } number & percent \\
\hline 0 & 1,965 & 32.4 \\
1 & 148 & 2.4 \\
3 & 374 & 6.2 \\
5 & 616 & 10.2 \\
10 & 448 & 7.4 \\
15 & 58 & 1.0 \\
20 & 531 & 8.8 \\
30 & 663 & 10.9 \\
40 & 617 & 10.2 \\
50 & 574 & 9.5 \\
60 & 34 & 0.6 \\
100 & 28 & 0.5
\end{tabular}

\begin{tabular}{ll}
\hline Total 6,056 & 100 \\
\hline
\end{tabular}

Number of bands

Average:

12

Standard deviation:

17.7
Rates in $\mathbf{2 0 0 0}$

\begin{tabular}{rrr}
\multicolumn{3}{c}{ Rates in 2000 } \\
\hline Bands & \multicolumn{2}{c}{ Number of lines: } \\
\cline { 2 - 3 } number & percent \\
\hline 0 & 2,029 & 32.0 \\
1 & 173 & 2.7 \\
3 & 381 & 6.0 \\
5 & 679 & 10.7 \\
7 & 7 & 0.1 \\
10 & 519 & 8.2 \\
12 & 2 & 0.0 \\
15 & 79 & 1.2 \\
18 & 1 & 0.0 \\
20 & 516 & 8.1 \\
25 & 3 & 0.0 \\
30 & 633 & 10.0 \\
40 & 678 & 10.7 \\
45 & 2 & 0.0 \\
50 & 569 & 9.0 \\
55 & 1 & 0.0 \\
60 & 12 & 0.2 \\
80 & 9 & 0.1 \\
100 & 48 & 0.8 \\
\hline Total & 6,341 & 100 \\
\hline & &
\end{tabular}

Number of bands

Average:

15.4

Standard deviation:

Source: Tariff schedule from the Ministry of Finance.

${ }^{1}$ The normal tariff rates ase termed preferential in the official schedule There are also nonpreferential tariff rates about 50 percent higher than these rates, which are applied to imports from countries without a trade agreement (or not in the process of negotiating one). 
APPENDIX

\section{ASEAN Free Trade Area (AFTA)}

At end 1995 Vietnam signed the AFTA agreement with the core commitment of reducing tariffs on almost all imports from AFTA members to 0-5 percent by the start of 2006. For the first five years Vietnam's AFTA commitments were met by applying low tariffs to goods that did not compete with domestic production. Beginning in 2000 , however, this liberalization is beginning to exert pressure on domestic enterprises to improve competitiveness.' Moreover, since AFTA members are in many cases low-cost producers, this process is likely to increase Vietnam's potential for manufactured exports by significantly reducing the costs of production and providing relatively strong international competition. Under the rules for AFTA, Vietnam is to submit a roadmap for meeting its commitments in 2001-06 by end 2000.

\section{Specific commitments}

The structure of Vietnam's agreement under AFTA is similar to the other members and is based on classifying the approximately 6,300 items on Vietnam's tariff code into four lists:

1. Inclusion List $(\boldsymbol{I} \boldsymbol{L})$ This list covers items on which tariffs are to be reduced to the common effective preferential tariff (CEPT) of 0-5 percent by 2006. It effectively comprises all items not on the exclusion lists below and will comprise about 6,030 items by 2003 . The IL currently comprises 4,233 items, of which tariffs on 1,270 items are over 5 percent and 451 items are over 20 percent, with tariffs on a few items as high as 50 percent. Although a linear tariff reduction over the phase-in period is recommended, the annual tariff rate reduction must be at least 5 percent. A comparison of the 1999 and 2000 CEPT schedules suggests that the most common reduction was 5 percentage points. Under the implementing arrangements, by 2003 very few $I$ items would have CEPT rates over 20 percent.

2. Temporary Exclusion List (TEL) This list covers items to be phased into the IL by 2003. In early 2000, in accordance with the existing plan, 640 items were moved from the TEL to the IL, to bring the IL up to 4,233 items. After the 2000 installment, the TEL now comprises about 1,800 items, which will be transferred to the IL in three roughly equal annual installments, so that by 2003 the TEL will be depleted.

3. General Exclusion List (GEL) This list covers items not subject to liberalization for cultural, security, health, and environmental reasons. This list comprises 202 items, including alcoholic beverages, automobiles, motorcycles and kits, and petroleum products, which are

\footnotetext{
'AFTA members accounted for 19 percent of Vietnam's exports and 28 percent of imports in 1999.
} 
currently subject to quantitative restrictions. However, the scope of the GEL is being reviewed, and a few items, including radios and larger motorcycles are likely to be removed.

4. Sensitive Exclusion List (SEL): In accordance with the Protocol on Sensitive and Highly Sensitive Products signed on September 30, 1999, Vietnam has 51 items, mainly unprocessed agricultural products and including sugar, on the sensitive list and no products on the highly sensitive list. Items on the SEL are to have tariffs reduced to $0-5$ percent by 2013 , and by at least 10 percent each year starting no later than 2006 , but sugar is specifically to reach the $0-5$ percent target by 2010 . All quantitative restrictions and other nontariff barriers are to be removed from these items by 2013 .

Vietnam: CEPT Tariff Rates, 1999-2000

\begin{tabular}{rrr}
\multicolumn{3}{c}{ Rates in 1999 } \\
\hline Bands & \multicolumn{2}{c}{ Number of lines: } \\
\cline { 2 - 3 } & number & percent \\
\hline 0 & 1,523 & 42.7 \\
1 & 82 & 2.3 \\
3 & 332 & 9.3 \\
4 & 1 & 0.0 \\
5 & 561 & 15.7 \\
7 & 20 & 0.6 \\
10 & 261 & 7.3 \\
15 & 470 & 13.2 \\
20 & 25 & 0.7 \\
25 & 24 & 0.7 \\
30 & 33 & 0.9 \\
35 & 3 & 0.1 \\
40 & 231 & 6.5 \\
45 & 1 & 0.0 \\
& & \\
\hline Total & 3,567 & 100 \\
\hline
\end{tabular}

Average:

7.1

Standard deviation: $\quad 10.6$

\begin{tabular}{rrr}
\multicolumn{4}{c}{ Rates in 2000 } \\
\hline Bands & \multicolumn{1}{c}{ Number of lines: } \\
\cline { 2 - 3 } & number & percent \\
\hline 0 & 1,690 & 39.9 \\
1 & 155 & 3.7 \\
3 & 335 & 7.9 \\
4 & 0 & 0.0 \\
5 & 783 & 18.5 \\
7 & 10 & 0.2 \\
10 & 573 & 13.5 \\
15 & 129 & 3.0 \\
20 & 107 & 2.5 \\
25 & 13 & 0.3 \\
30 & 72 & 1.7 \\
35 & 280 & 6.6 \\
40 & 82 & 1.9 \\
45 & 1 & 0.0 \\
50 & 3 & 0.1 \\
\hline Total & 4,233 & 100 \\
\hline
\end{tabular}

Average:

7.3

Standard deviation:

10.7

Source: Tariff schedule from the Ministry of Finance.

\section{Nontariff barriers}

Under the AFTA agreement, QRs for the IL items should be removed at the latest by the time the import: (i) satisfies the AFTA content requirement of at least 40 percent; (ii) is on the IL of both source and destination country; and (iii) has a CEPT rate of 20 percent or less. In practice, Vietnam has removed QRs on AFTA imports sooner, and on a multilateral basis, typically when the item was moved from the TEL to the IL. Other nontariff barriers are to be removed from the goods within five years from when these three conditions are met. 


\section{H. Recent Developments in the Foreign Exchange System}

\section{Recent developments in the foreign exchange market}

Since the early 1990 s, following the unification of exchange rates, exchange rate management has been characterized by periodic adjustments to the band within which the official exchange rate was allowed to move. From August 1998 to February 1999, the interbank exchange rate of the dong was subject to a maximum 7 percent depreciation limit from the fixed official rate vis-à-vis the US dollar. In practice, the interbank exchange rate remained at the lower limit during this period. In February 1999, a new system was introduced under which an official exchange would not be set, but an interbank market rate would be quoted daily, calculated as the weighted average of rates in the interbank market during the previous day. However, the value of the dong in the interbank market is not allowed to depreciate by more than 0.1 percent compared to the previous day. This new system was aimed at gradually moving toward greater exchange rate flexibility and giving more weight to market forces in exchange rate determination.

Since the introduction of the new exchange rate regime, movements in the rate have been marginal. Between February 1999 and June 2000, the dong depreciated in nominal terms by only 1 percent vis-à-vis the US dollar. This stability was aided by the strong export performance (see Note III) coupled with stagnant imports, and larger inflows due to measures taken in 1999 to encourage remittances by Vietnamese overseas.

However, thete have also been pressures on the dong. For example, because of the lower interest rates on dong deposits (which were reduced by 6 percentage points in 1999 to 4 percent by year-end) at a time of rising U.S. dollar interest rates, the interest rate differentials on deposits increased sharply, causing a portfolio shift toward foreign currency deposits. The rapid expansion of credit since late 1999 is also likely to increase the demand for foreign exchange. Also, the relaxation of the foreign exchange surrender requirement from 80 percent to 50 percent in August 1999, at a time when some participants in the exchange market were experiencing delays in obtaining foreign exchange, was accompanied by temporary pressure on the dong from some hoarding of foreign exchange.

Information from the authorities on the parallel market exchange rate shows that deviations from the interbank rate were less than 2 percent in 1999. Although the reported parallel market exchange rate in Vietnam is not a fully representative market rate, in earlier years it had provided some signals on whether the interbank rate was notably misaligned. The value of transactions in the officially quoted parallel market is smalI, estimated at US\$1- 5 million a day, while the size of the interbank transactions is estimated at about US\$40-50 million.

Official intervention policy in the exchange market aims at maintaining export competitiveness, managing imports, and smoothing out short-term exchange rate fluctuations. However, in practice this policy has contributed to the stability of the dong visà-vis the US dollar. The State Bank of Vietnam (SBV) has also intervened at times when there was uncertainty in the market and when it considered that the supply and demand for 
foreign exchange did not reflect underlying market factors. For example, intervention took place in February 1999, when the new exchange rate mechanism was introduced, and in the latter part of the year on account of concern over the millennium bug.

\section{Recent developments in exchange restrictions}

Although foreign exchange restrictions have recently been eased, the system is still considered restrictive. In particular, there are three restrictions on payments for current account transactions which are subject to approval under the Fund's Articles of Agreement, Article VIII, Sections 2, 3, and 4).

1. Foreign exchange balancing requirement is imposed on foreign-invested companies. It was introduced at a time of foreign exchange shortages, requiring foreign-invested enterprises to balance their foreign exchange needs with their foreign exchange receipts, as stipulated in the Foreign Investment Law (FIL). Although this restriction has become effectively less binding, affecting apparentiy only a small proportion (20-30 percent) of foreign-invested companies, it remains a source of concern for foretgn investors. ${ }^{2}$ In May 2000 , the FIL was amended, resulting in a significant relaxation of the foreign exchange balancing requirement for foreign-invested enterprises and parties to business cooperation contracts. Most classes of foreign-invested enterprises are now permitted to buy foreign exchange from commercial banks, subject to the same regulations as domestic enterprises (see below). However, in some cases where the foreign investment is in projects considered by the government to be important, including for infrastructure works, the government will guarantee the availability of foreign exchange. Implementing regulations, which are required to make this change effective, have not yet been issued.

2. The amendment to the FIL also reduced the rates on the tax on profit remittances. The new tax rates are 3,5 , and 7 percent, compared to the previous rates of 5,7 , and 10 percent. This tax, which applies to the repatriation or remittance of profits by foreign enterprises (and not on profit itself), continues to give rise to a multiple exchange rate practice and constitutes another exchange restriction.

3. In addition to these explicit restrictions, there are other administrative measures and controls that limit the foreign exchange available for imports of certain goods, depending on the foreign exchange situation. For example, importers of consumer goods are required to supply their own foreign exchange for imports and are only allowed to use

\footnotetext{
${ }^{2}$ The SBV indicates also that the balancing requirement could be avoided given that foreign enterprises could always pay Vietnamese companies in dong to import on their behalf, but there are likely to be additional costs from this operation. Foreign-invested enterprises approved for the production of import substitutes have generafly been exempted from the balancing requirement and are permitted to buy foreign exchange in the market.
} 
immediate payment methods. ${ }^{3}$ In addition, "non-priority" goods have been constrained by the availability of foreign exchange at the commercial banks, which contributed to the low level of imports in the past three years.

During 1999 , there was no evidence that the nonavailability of foreign exchange contributed to the incurrence of external payments arrears. External arrears identified in the balance of payments were only incurred on the transferable ruble debt to Russia, which is under active negotiation. However, foreign-invested enterprises rescheduled almost half of their debt service obligations to parent companies and foreign commercial banks, due mainly to financial difficulties and not related to exchange restrictions.

\section{Conclusion}

The recent relaxation of exchange controls and the relative stability of the exchange rate are encouraging, but further reforms of the exchange arrangements are needed to boost confidence and attract foreign direct investment. In particular, the foreign exchange balancing requirement and the tax on profit remittances by foreign-invested firms should be removed. Also, the difficulties and delays in obtaining foreign exchange from commercial banks should be overcome by further widening the band for movements in the interbank exchange rate to allow the rate to better reflect demand and supply conditions in the market. Elimination of the surrender requirement would also help in broadening the foreign exchange market.

\footnotetext{
${ }^{3}$ The most recent renewal of this control is in the Decision on the Management of Import and Export of Goods in the Year 2000 (Decision 242/1999/QD-TTg), promulgated in December 1999. There are also specific restrictions on the import of commodities such as gold and other precious metals where import licenses are granted only if importers have the required foreign exchange.
} 


\section{Recent Export Performance}

\section{Export performance in 1999 and early 2000}

The growth rate of Vietnam's exports in 1999 outperformed that of most Asian countries. After a weak performance in 1998 , exports rose sharply by 23 percent (to US $\$ 11.5$ billion) a rate comparable to those prevailing in the mid-1990s. Performance in the first half of 2000 continued to be strong, rising by an estimated 25 percent over the same period in 1999. Three main factors have contributed to this strong performance in 1999:

- A surge in the value of crude oil exports by 62 percent due equally to increases in both price and volume, accounting for slightly more than one-third of total export growth. The bulk of oil exports continued to go to the Asian region.

- A recovery in the Asian region, which also led to increased demand for nonoil exports from Vietnam.

- An increase in nonoil exports to the European Union (in particular garments and footwear) which benefited from more favorable access to the area in 1999.

Over the medium term, exports are expected to continue to grow, especially with more robust growth in nonoil exports, assuming cautious macroeconomic policies, an acceleration of structural reforms, and the liberalization of exchange and trade arrangements.

Vietnam: Contribution to Export Growth by Commodity and Region (In percentage points of annual export growth)

\begin{tabular}{lrrr}
\hline & 1997 & 1998 & 1999 \\
\hline Total exports 1/ & & & \\
Oil exports & 24.6 & 2.4 & 23.2 \\
Nonoil exports & 1.0 & -2.0 & 9.2 \\
$\quad$ Asia \& Pacific region & 23.6 & 4.5 & 14.0 \\
European Union & 10.5 & 3.0 & 4.7 \\
United States & 15.2 & 3.2 & 5.2 \\
Other & 1.0 & 1.2 & 0.4 \\
& -3.0 & -2.9 & 3.8 \\
\hline
\end{tabular}

1/ Annual percentage change.

Regional demand for nonoil exports from Vietnam is also expected to remain reasonably strong, given the projected continuation of the regional recovery. Staff projections suggest that real GDP growth in most countries in the region would be in the range of 4-6 percent in 2000 , with the average export-weighted real GDP growth rate for the Asian region of about 5 percent per year during 2000-02. 
However, given the volatility in oil prices and the large share of crude oil in total exports (17.5 percent in 1999), and the continued reliance on other traditional exports (i.e., coffee and rice), the export base will remain vulnerable to terms of trade shocks if structural reforms and private sector development-which aim in part at diversifying this base over the medium term-do not materialize. ${ }^{4}$ In addition, the possible loss of competitiveness in other sectors such as garments and footwear due to higher production costs associated with continued heavy regulations would put at risk this medium-term outlook.

\section{Export market shares}

In the last few years Vietnam appears to have gained some market share in major export markets. Vietnam's relative performance in the markets of Japan, other Asia, western Europe, and the United States was compared with imports from a group of competitor countries (Thailand, Indonesia, Philippines, and China) in 1996-98. Vietnam has lost only marginally its market share in Japan, and made some small market gains in other markets with a noticeable increase in Europe. Imports from the Philippines and China increased the most in these markets at the expense of imports from Thailand and Indonesia. The relatively large increase in Vietnam's market share in Europe mainly reflects increases in quotas and improved market access.

\section{Real effective exchange rates}

Using recent trade weights, Vietnam's real effective exchange rate (REER) appreciated by about 3 percent since June 1997. The REER with respect to Asia (excluding Japan) appreciated by 8 percent in the period since June 1997, largely due to the sharp depreciation of the nominal exchange rates of its regional trade partners, and by 4 percent against main European partners. By contrast, the dong depreciated by 14 percent in real terms with respect to the dollar and the Japanese yen. Compared to the REERs of selected Asian countries, Vietnam's REER has appreciated, while others have depreciated since the onset of the Asian crisis (see chart).

Notwithstanding these developments, caution is required in using the REER as a measure of export competitiveness, especialiy in an economy such as Vietnam. To the extent that there are administered prices (such as petroleum products), ceilings on bank lending rates, and subsidized production (as with loss-making SOEs), the REER is likely to overstate Vietnam's competitiveness. In addition, the presence of significant trade and exchange restrictions means that it is harder to determine an equilibrium exchange rate to serve as a benchmark for comparisons. Also, the equilibrium REER can change over time due to structural changes in both the home and directly competing countries

\footnotetext{
${ }^{4}$ In addition, at current levels of production, a US\$5 decline in the average per barrel price of crude oil would likely lower exports by US\$0.5 billion, or 5 percent of total exports.
} 
REAL EFFECTIVE EXCHANGE RATES

(June $1997=100$ )
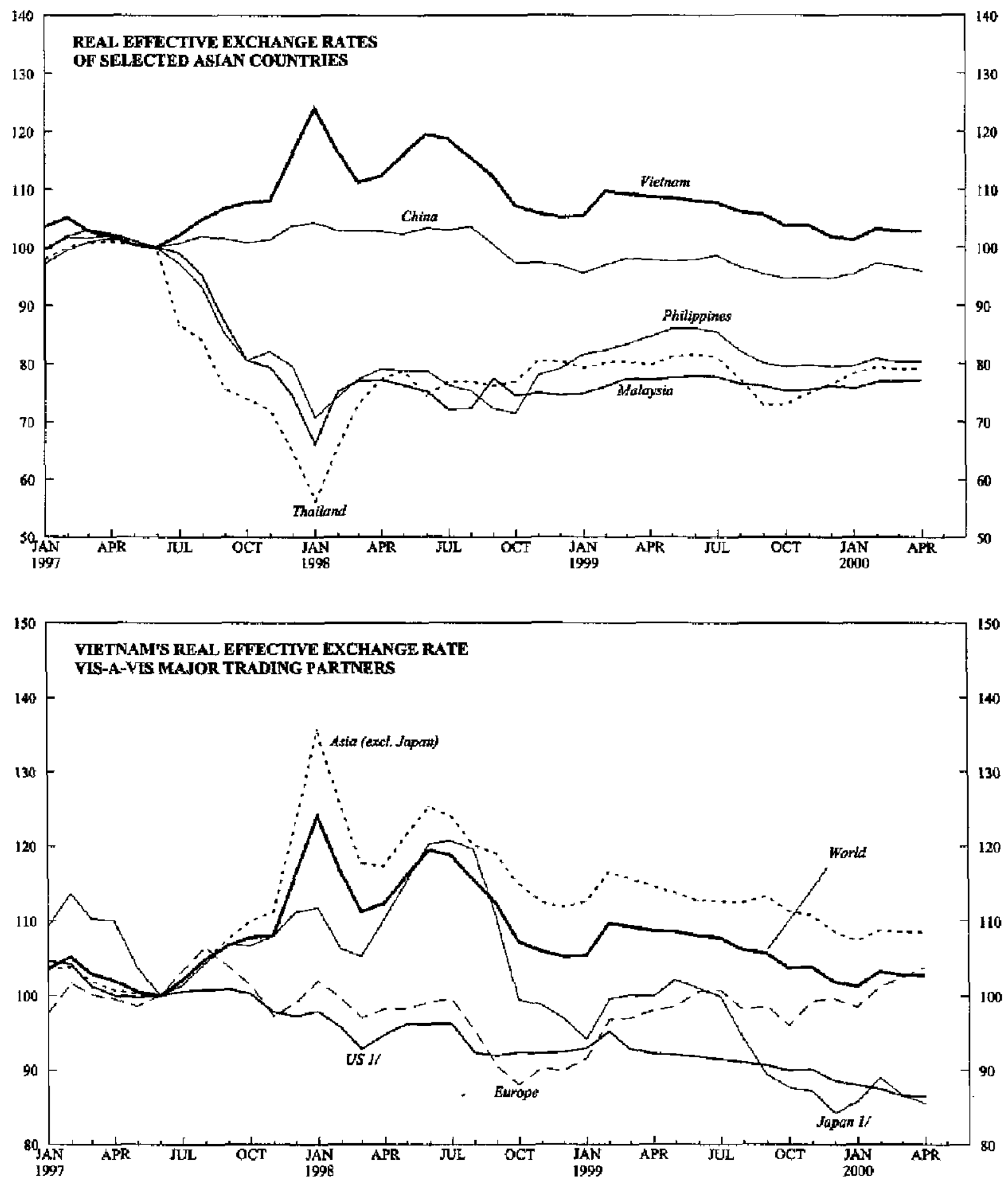

Sources: IMF Information Notice System, the Vietnamese authorities, and Fund staff estimates.

I/ Bilateral rtal exchange rates. 


\section{TV. Summary of Current Poverty Situation ${ }^{\text {s }}$}

In recent years, Vietnam has made considerable strides in reducing poverty, but it still remains one of the poorest countries in East Asia. During the period 1992-99, real per capita income (in dong) increased by an average of 5.8 percent a year (staff estimate). However, in 1999, per capita GDP was still only US\$372. Based on the 2000 World Development Indicators, per capita GNP in Vietnam was in the bottom quintile (of the 138 reporting countries) in 1998, and was only about one-third the level recorded in the low and middle income countries of the East Asia and Pacific region.

According to the latest Vietnam Living Standards Survey (1997/98), conducted by the General Statistical Office (GSO) with technical assistance from the World Bank, the share of persons living below the poverty line in Vietnam fell from 58 percent in 1992/93 to 37 percent in $1997 / 98 .^{6}$ The Attacking Poverty report attributes this decline in poverty to high growth rates, despite a rise in inequality that occurred during this period. ${ }^{7}$

However, it cautions that recent dramatic gains in poverty reduction remain quite fragile. In particular, because a large number of persons were positioned close to the poverty line in 1993, modest improvements in income and employment

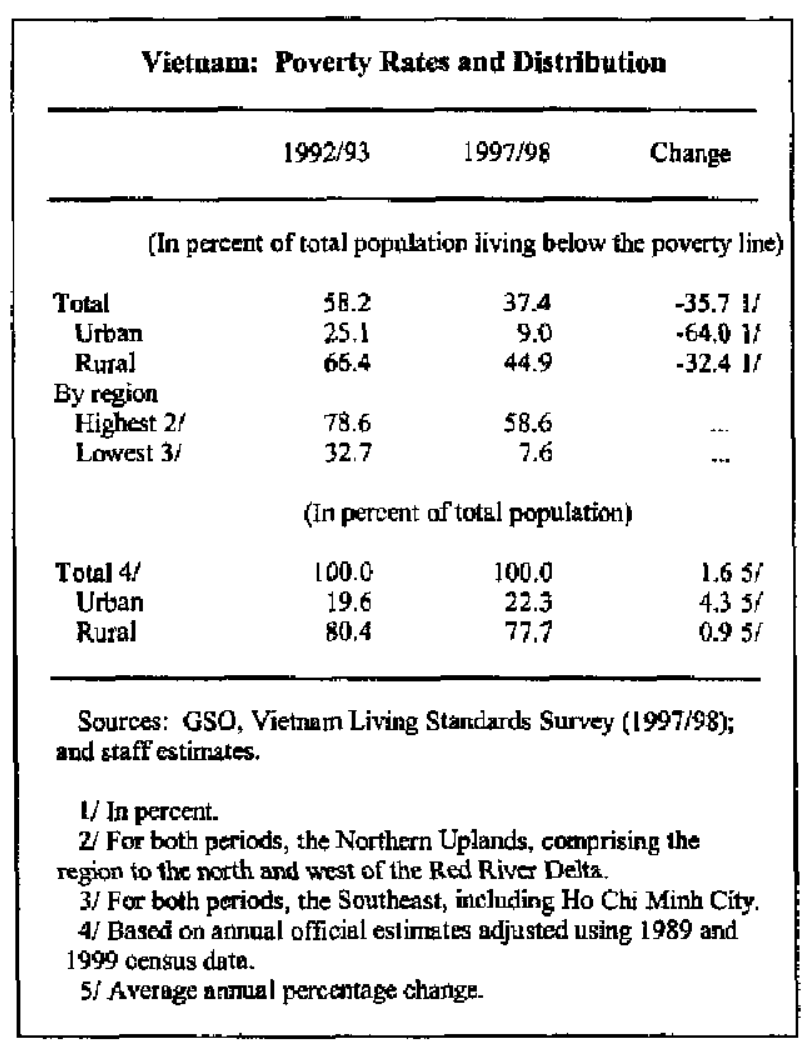

${ }^{5}$ This section draws heavily from the joint government, donor, and NGO report titled Vietnam Development Report 2000: Attacking Poverty (1999), which was prepared under the guidance of the World Bank in conjunction with the December 1999 Consultative Group meeting.

${ }^{6}$ The level of food poverty, defined as persons with expenditure lower than the cost needed to obtain daily food equivalent of $2,100 \mathrm{kcal}$, also declined significantly over this period, from 25 percent to 15 percent.

${ }^{7}$ If inequality had not increased, the report notes that Vietnam would have been able to reduce poverty by an additional 8 percentage points. 
opportunities have been sufficiently strong to pull them over the poverty line so that they are now bunched just above the line. ${ }^{8}$. It follows that the slowing of economic activity experienced over the past few years may have pushed a number of these persons back below the line, since per capita GDP growth is not evenly distributed.

Based on the most recent living standards survey and as discussed in the Attacking Poverty report, the current poverty situation in Vietnam can be summarized as follows:

- Poverty is largely a rural phenomenon in Vietnam, with 45 percent of the rural population living below the poverty line in 1997/98. Farm incomes have risen substantially, but farm employment has not risen owing to productivity gains. Therefore, the generation of nonfarm rural employment remains a major

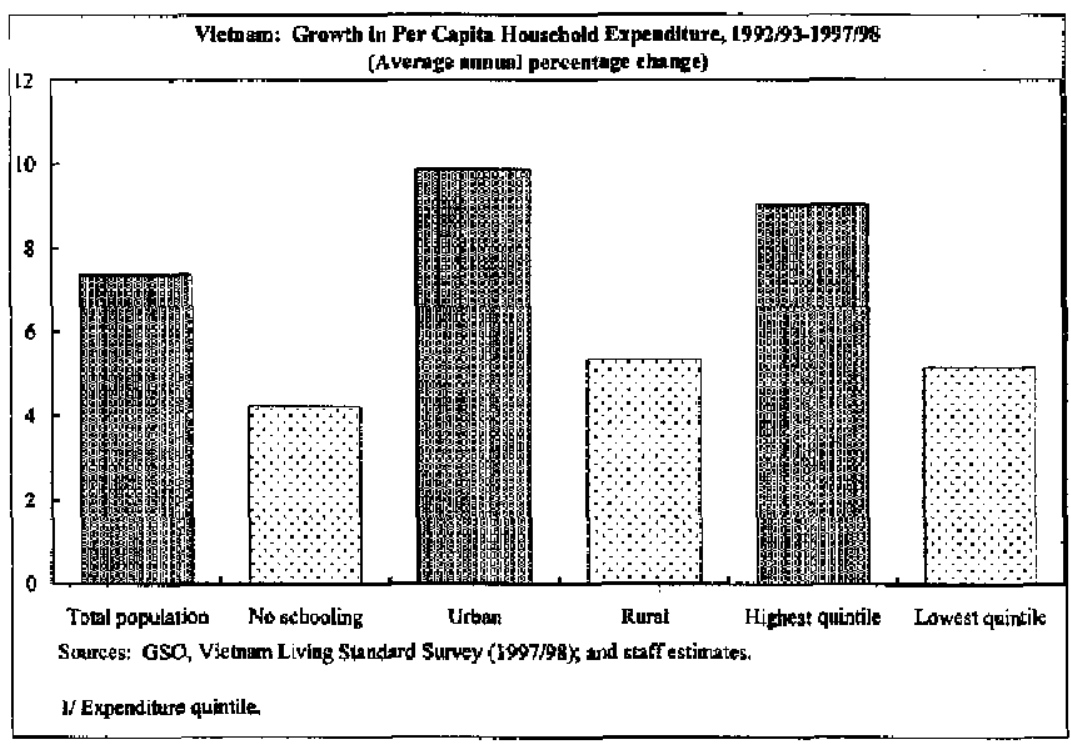
challenge.

- Urban poverty, while comparatively small, may be a particularly complex phenomenon, as among other things the creation of new employment opportunities in urban areas appears to be more closely linked to the implementation of structural reforms and improvements in the investment climate.

- Equity is primarily a urban-rural issue in Vietnam, but is also an issue of concern across regions and gender. Inequality in access to education may be one factor explaining intracountry differences in poverty patterns.

- The vulnerability of the poor to household-specific and system-wide shocks (both internal and external) appears to be greatest among rural households, which poses a major challenge to poverty reduction efforts.

\footnotetext{
${ }^{8}$ According to World Bank estimates, the incidence of poverty likely fell in the range of 30 45 percent of total population in 1998.
} 
- Moreover, fornal safety net coverage is low and not well targeted. Most migrants to urban areas, who tend to be poor, lack permanent registration status and thus face greater difficulties in accessing public services.

The Attacking Poverty report identifies several factors that contributed to the significant reduction in poverty in the mid-1990s. In rural areas, where agriculture remains the dominant source of income and employment, the rural living standard benefited from more diversified and rising agricultural incomes. In particular, farm households growing industrial (mainly cotton, jute, rush, sugarcane, peanuts, soybeans, and tobacco) and perennial (tea, coffee, rubber, coconut, and pepper) crops experienced the largest revenue gains. Rapid growth also was experienced in household nonfarm employment

\begin{tabular}{|c|c|c|}
\hline \multicolumn{3}{|c|}{ Vietnam: Education Standards } \\
\hline & $1992 / 93$ & 192798 \\
\hline $\begin{array}{l}\text { Literacy rate } 1 / \\
\text { Urtan } \\
\text { Rural } \\
\text { Male } \\
\text { Fernale }\end{array}$ & $\begin{array}{l}93.3 \\
84.8 \\
91.4 \\
82.3\end{array}$ & $\begin{array}{l}94.1 \\
88.0 \\
93.7 \\
85.6\end{array}$ \\
\hline \multicolumn{3}{|c|}{ 6-10 yearsald Allschool ages } \\
\hline $\begin{array}{l}\text { Urben } \\
\text { Rural } \\
\text { Pootest quintile } \\
\text { Richest quintile }\end{array}$ & $\begin{array}{l}95.9 \\
92.0 \\
84.8 \\
96.8\end{array}$ & $\begin{array}{l}63.5 \\
44.1 \\
31.4 \\
70.0\end{array}$ \\
\hline \multicolumn{3}{|c|}{$\begin{array}{l}\text { Source: GSO, Vietnam Living Standards Survey } \\
(1997 / 98) \text {. }\end{array}$} \\
\hline \multicolumn{3}{|c|}{$\begin{array}{l}\text { 1/In percent of population } 10 \text { years and older. } \\
2 / \text { In percent. }\end{array}$} \\
\hline
\end{tabular}
in rural areas (mainly retail trade and food processing) - by an average of 6.7 percent a year during 1993-98, compared with growth in household farm and wage employment of 0.8 percent and 3.3 percent, respec-tively. Overall, rural employment increased by an average of 1.7 percent a year during 1993-98, compared with rural population growth of about 1 percent a year, suggesting that factors such as family planning and rural-urban migration may also have been factors in explaining rural poverty reduction. In addition, underemployment declined significantly, but it remained much higher in 1998 in rural (61 percent) in comparison to urban ( 40 percent) areas. ${ }^{10}$

Rice-the main staple of Vietnam-remained the dominant source of farm income (43 percent of total agricultural revenue in 1998). However, between 1993 and 1998, real per household revenue derived from the sale of rice rose by only about 4 percent a year, considerably lower than real GDP growth. Among factors identified as limiting income generating opportunities in the agricultural sector are continued restrictions on land use, lack of access to high quality seeds and adequate fertilizer, and existence of a rice export quota. Moreover, agriculturally-oriented state-owned enterprises still exert some control over input, output, and export marketing decisions, which limit farm income growth.

\footnotetext{
${ }^{9}$ However, 85 percent of rural nonfarm enterprises employ just one or two workers, with three-quarters of these enterprises also engaged in other forms of employment.

${ }^{10}$ In Vietnam, unemployment rates are not a very useful indicator of labor market changes, since most people are engaged in some form of activity and open unemployment is rare (except in urban areas).
} 
In urban areas, poverty reduction has also derived from growth in nonfarm and wage employment, although each was roughly at the level of urban population growth during 1993-98, which averaged about 4 percent a year. However, owing to a large drop in urban household farm employment, total urban employment increased by only 2 percent a year. Therefore, factors explaining the drop in urban poverty are less conclusive than those explaining the drop in rural poverty. It should be noted that the latest living standard survey is likely to have underestimated the level of urban poverty by excluding those migrants who do not have a right to permanent residency in urban areas. ${ }^{1 \mathrm{I}}$

Despite the impressive rise in industrial production during this period (on average by about 13 percent a year), employment in this sector grew at less than 4 percent a year. Industrial

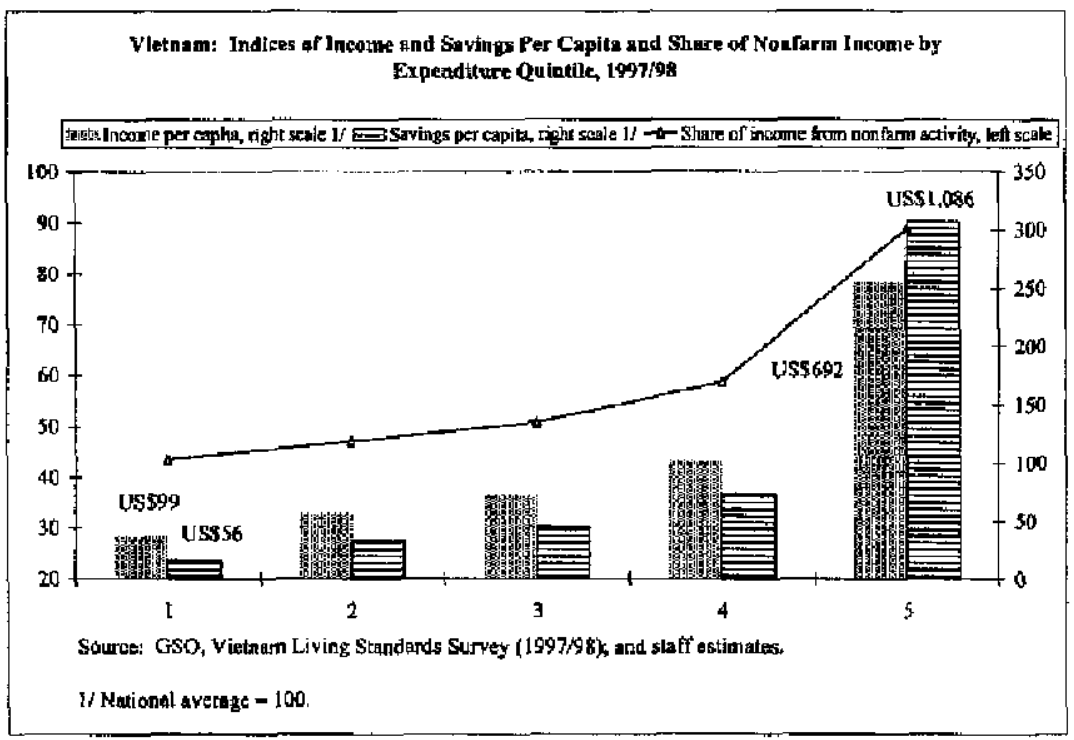
enterprises, in which the state sector is dominant, tend to be capital intensive and heavily protected and, as a result, more oriented toward import substitution. Moreover, many of these enterprises have become loss-making as they have been exposed to more competition. The Attacking Poverty report found that the capital intensive and enclave nature of industrial production in Vietnam tended to minimize its effect on employment creation in the rest of the economy.

The Attacking Poverty report concludes that the level and pattern of growth in the future will determine the pace of poverty reduction, noting that the importance of economic growth in raising living standards of the poor in Vietnam cannot be overestimated. The pattern of growth, however, will not likely to be able to replicate the land reform-based, agricultural diversification growth experienced in the early and mid-1990s, which is now reaching its constraints. Thus, the report notes that the real challenge will be on the creation rural nonfarm and urban employment, with the creation of conditions for new small- to mediumscale enterprises playing a significant role to this end.

"Adjusting for unregistered urban migrants, the World Batk estimates the incidence of urban poverty was in the range of 10-15 percent in 1998. 
1. I. Personal Ineme Tax

(a) Regular income (in cash or kind):

- salaries and wages

- allowances

- bomuses

Bxcluded are:

- interest income

- reftal income

- social benefits

remuneration for technical innovation'inverticon - travelling expenses, allowances for shift work, income eamed in thoxious ot dangerous conditions or in mountainous areas and stiputated offshore

istands

- dividend

\section{(b) Iregular income}

- gifts in kind from overgeas

- lottery prizes

- fees or commissions for technolog

transfer, industrial design or technieal

\subsection{Supplementary Income Tax}

Levied on monthly income above VND8 million net of regulat iteothe tax payzinent

\subsubsection{Enterpise Gneorne Tax}

Faxable income defined as the difference between the total revenue and total expenditure of the enlerprise, together with any other income such as rents, proceeds of liquidation or transfor of assets, profits enmed from business operation abroad, and profits from financial dealings.
Individual invertors ane exempted for 5-10 years from income tex applicable to incothe eattred by contributing eapital and purchasing tharem in business

Investoss ate exompled from income tex applicable to income earned by contributing capital through intellectral property tights, technical know-how,

or technological process

Non-citizens are exempted.

For chenieals, ccnetruction (excl. cement), trangportation (excl. air and taxi),

miniting and stecl production 25\% rate until 2002 (1/1/2002)

For oil sector, and requurces exploitation sectors 32-50\% at govennent discretion For other resources exploitation seclors 32-50\% docided by MOF or Foreign Invostment License issuing authority with the agreement of MOF.
$30 \%$ of excess income

\begin{tabular}{cc} 
For citizens: & $\begin{array}{c}\text { grass rate } \\
\text { monthlyincome [XND mil) }\end{array}$ \\
\hline $0-2$ & CND +\% over floor \\
$2-3$ & exempt \\
$3-4$ & $10 \%$ \\
$4-6$ & $20 \%$ \\
$6-8$ & $30 \%$ \\
$8-10$ & $40 \%$ \\
$10+$ & $50 \%$ \\
& $60 \%$
\end{tabular}

For rusident forejgners, Vietnamese on mission or working oversen:

monthly income CVND mil

$\begin{array}{cc}0-8 & \text { exompt } \\ 8-20 & 10 \% \\ 20-50 & 20 \% \\ 50-80 & 30 \% \\ 80-120 & 40 \% \\ 120+ & 50 \%\end{array}$

For non-resident foreigners: flat rate $10 \%$

For citizens and resident foreigners. transaction income (VND) mil)

$0-2$
$2-4$
$4-10$
$10-20$
$20-30$
$30+$

Gifts in kind from overseas: 1lat 5\% (nil below 2 million) Lotfery wirnings: flat 10\% (nil below 12.5 million) Ineome from technology transfers: flat $5 \%$ (nil below 2 mil.)

$32 \%$ standard rate

$50 \%$ oil sector standard rate

Co-operatives, households whose income are gained by agricultural activities and products value are less than 90 million dong per year or income less than 36 million 
V. Vietnam: Summary of the Tax System as of January 1, 2060

Expenditures deductible from toverue:

- costs of raw and othet materials and energy

required for the manufacture of principal products

and by-products or for the provision of services

- Fages, salaries, allowances

- depreciation of fixed assets

- costs of research and development

- costs of achuigition or of the right to use any

technisal documionts, patents, or licenee, and

costs of techrical service

- enterprise matajagenent experses

- taxes (other than profit tax or profit remittance tax)

- interest payments (subject to certain liznitations)

- costs and expenses for packaging, distribution

and torage

- costs of insuring the assats of the enterprise

- any losges brought forward from previous years (max 5 )

- social insurance expenses

- expenses incurred for employee sofety

- severance payments

- overhead cost allocations

- other expenditures not exceeding $5 \%$ of the total

amount of the above expenditures
Exemption or reduced rate for estabishment of production, for BTO or BOT contracts, investment projects in regions with difficult or particulatly diffieult economic and social conditions, and following investment projects:

- afforestation and forest pianting

- infrastructure and public trantispor

- education, lealth case, culture

- export production and trading

- agricultural and martitime development

- science, business management, technology transfer

- produstion reform or relocation

- developing preferential professions

Investors of export products production entities and export procuets trading establishments receive additional reductions as followg:

- $50 \%$ reduction in first year when export a new product, or when export to a new market

- $30 \%$ reduction in the year when export tumover exceeds that the previous year $-30 \%$ reduction in the year when export ermings exceed $50 \%$ of turnover, or in the third consteutivi year of stable export market

- additionsl $25 \%$ reduction of the tax on income from exports in the above-listed cases if projects in regions with difficult economic and social conditions

- exemption from the tax on inconc from exports in the above-listed cases if profects in regions with particularly difficult economic and social conditions

1.3.2. Enterprise Income Tax on Foreign Enterprises and Joint Ventures

As 1.3 .1 .

\section{Tax holidays:}

Joint ventures and wholly foreign-owned companies nizy be

considered for tax holidays. The holidays take the form of a cormplet

exemption from profit tax for a certuin period beginning inumediately

after the project bacomes profitable, followed in turn by a period where tax is

charged at half the agreed preferential rate. The duration of holiday

periods depends on tax rate applicablo to project as follows:

(a) projects subject to standard nate may be considered for max, two years

exernption if investment made for production in rural areas

(b) project subject to $20 \%$ rate may be considered for one year's

exemption, followed by up to two years at $10 \%$ rate

(c) projects aubject to $15 \%$ rate may be considered for two years"

exenation followed by up to three years at $7,5 \%$ rate.

(d) projects subject to $10 \%$ rale may he considered for four yean'

exemption, followed by up to four yeers at $5 \%$ rate. Afforestation or

inffastructore projects in mountainous or nemote ajeas and projects with large-

scale socio-economic inpast in the special investment etcouragenent list obtain exemption for 8 years after becoming profitable

(e) projects in irvestment encouragement areas and investment oncouragement

special hist obtain exemption for 4 years and $50 \%$ reduction for the next 4 year
Standard rate $25 \%$

Oil $50 \%$

Proferentisl rates of $20 \%, 15 \%, 10 \%$ available for inward investment:

1. Following the granting of investment license, the

following rates are available for $10-15$ years:

A. $20 \%$ for 10 yenrs

for projects meeting at least ane of the following criteria:

(a) more than 500 employees

(b) advanced tectimology uged, investment in R\&D

(c) at least $50 \%$ of production for export

(d) grow or prosess agrieultural, forest, or a gua products

(e) use Vietnamese raw malerial

B. $15 \%$ for 12 years for projects meeting at least ons of the following criteria or thin of the criteria in $A$

(a) at least $80 \%$ of production for oxport

(b) infrastructure construction and operation projects

(c) heavy indugtry such as refining of metals, production

of primary chemicals, refined oil products, fertilizers, 
v. Vietnam: Summary of the Tax System as of January 1, 2000

If profita ane reinvested in the business for at least three years, the tax paid on profite sa reinvested may be refundect. Such profits, when eventualty repatriated, are subject to profit remittance tax.

Enterprises in "ind ustrial zones" are entitled to the following tax incentives: (a) production enterprises exporting less than $50 \%$ of production have tax rate of $15 \%$ with tax excmption for 2 years after having taxable income

(b) production enterprises exporting $50-80 \%$ have the tale of $7.5 \%$ with lax oxemption for tro years after becoming profitable

(c) production enterprises exporting mors than $80 \%$ have $10 \%$ rate, with excuption for 2 ycars after having taxable income and $5 \%$ tate for the following two years

(d) service enterprises have $20 \%$ rate, with exemption for othe year after having taxeble insome

(e) a company developing infrastructure of an industrial zone has $10 \%$ rate, with exemption for 4 years after having taxable income, and $5 \%$ rate in following 4 years

Enterprises operating in "export processing zonis" are entitled to the following tox incentives:

(a) $10 \%$ rzte for protuction and $15 \%$ for sevice enterprises

(b) exemption for the first two years atter having tarable income for sivvice and four years for production enterpciges

Foreign investors fully contributing logal capital and roinvesting their earning into projects included in the list of encouraged projects for at least 3 years are refunded the profit lax paid on reinvented eamings. The refund rate is :

(a) $100 \%$ for projects subject to $10 \%$ tax rate

(b) $75 \%$ for projects subject to $13 \%$ tax rate

(c) $50 \%$ for projects subject to $20 \%$ tax rate

1.3.3. Enterprise Income Tax on Foreign Contractors Based on value of contract with Vietnamese party

\subsection{Excess Income $T a x$ \\ Levied on after-tax income in excess of 12 percent of the owners' capital}

1.5. Withholding Profit Remittance Tax Income eamed by any way is taxable when remitted to foreigr countries.

\section{Capital Gains Tax}

Taxable gain is defined as the transfer price less the original cosk of the transferred oapital and expenses associated with the transfer
Exempted are foreipn companies as well as preferential itrestmenth ptojecto ant projects in regions with difficult or particularly difficult exonornic and social conditions

For enterprises in "industrial zones" andi "export processing zones", tax rate $5 \%$ BOT, BTO or BT enterprises: tax ratc is $5 \%$

Transfer to State-owned enterprises where State shares are dominant: exempt Transfer to other Vietnamese enterprises other than above onea: reduction of $50 \%$ tax rate

Expatriates: excempt and heavy metal engineering; car component, motorbike and efectronic items production

(d) planting of perennial indurtrial trees

(e) projects in areas under difficult natural, socio-econonic conditions

(f) projects in which the foreign party undertakes to transfer the assets to Vietnam for nil cansideration at the end of the operating period (ineluding hotel projocts)

C. $10 \%$ for 15 years for projects in areas under difficult conditions

(a) infrastructuro projects in areas under difficult naturat, socio-enonomic conditions

(b) investment in moumtsinous and remote areas

(c) afforestation projects

(d) projects approved under the BOT, BTO, BT regulations. (10\% for whole periòd, exeept first 4 profitable years exempted and following 4 years $5 \%$ If project is on the investruent encoura gement list. exemption first 8 profitable years)

(e) projects in the special investment encouragement list

Preferentisl tax rate shall be applied duritg the whole duration of projects that are on the invesiment encouragement list or on the investment encouragement special jist.

1. $1 \%$ for trading businesses

2. $2 \%$ for transportation, construction and production

$3.5 \%$ for services

4. $10 \%$ for profit fiom loan and copyright.

$25 \%$

1. $5 \%$ where foreigan investors contribute legal capital of more than US\$10 million

2. $7 \%$ where foreign investars contribute legal capital

more than USS5 million

$3.10 \%$ in all other cases

$25 \%$

CInternational Monetary Fund. Not for Redistribution 


\section{Taxes on Goods und Service}

\subsection{Value-added $\mathrm{Tax}$}

For imparts, base includes the import duty

- Goods and services for the puppos of production, business and consumption in Vietram.

- For domestic production-buainegs goods, tax is put upot before-VAT price.

\subsection{Special Consumption Taxes (Excises)}

Tax base is the tax-exclusive sale price, except for domentically produced alcohol, easino business, jackpot machines, and golf course businese, for which the taxable price is specificnlly stiputated by govenumeut.
Until 2002, maximum neduction of 100\% if VAT liability exceeds turnover tax liability and caumes losses

\section{$30 \%$ reduction for trading of excisable goods, engines and machinery}

Regulations on 27 groups of goods not subject to VAT (below)

(i) agricultural production

(ii) excisable production and imports

(iii) transfer of land use rights

(iv) credit services

(v) certain insurance services

(vi) medical services

(vii) teaching and training

(viii) printing and publishing (newspapers and certsin types of publications)

(xi) bus transport

(x) goads in transit

(xi) techinology transfer

(xii) inported fixed assets

(xii) goods and services for intemational transport and consurners abrosd

(xiv) goods and services by businesses below threshofd

(xw) foreign charity and grants, and related imports

(xwi) temporary irtuports for re-exports

(svii) materials for export production

(sviii) goods and services for international traffic

(kix) goods sold al duty free shops

(xx) unprosessed gold

(xoi) arma for nationnl defense

(xxii) state-owned houses sald to tenants

(xxiy) non-business euluural and sports activitios

(xxiv) staie-funded brosdcasting

(arv) public sanilary, drainge, and park services

(xovi) geologieal investigation and surveys

(xsvii) inigation

Not subject to special consumption tax;

(a) goods diractly exported by production or processing enterprises

(b) goads imported in the following cases:

- humanitarian aid, nor-refundable iid programs, donation to atate agencies, political organizations, socio-politícal organizations, social organizations,

socio-occupational organizations, units of amiad forets diplomatic immunity,
0\%: Exports

5\%: Provision of essemtial goods and services (e.g. slean water, fertilizers, foodstufts, medicines, various agricultural goods and services)

$10 \%:$ "Stutidatd rate"

(e.g mineral prods, power, electrical products, proesed food, construction, assembly, post and transportation)

20\%: Trading in precious minerals, lotteries.

CInternational Monetary Fund. Not for Redistribution 
V. Vletnam: Summary of the Tax System as of January 1, 2000

For imported goods, the taxable price is the import dutiable price plus the infort duty.

\section{(a) cigarettes, cigars}

(b) beer

(c) alcoholie beverages other than beer

(d) cars

(e) gasolite

(f) air conditioning equipment (below 90,000 bitu)

(g) playing oards

(h) votile paper and products

(i) massagc, karaoke, danco-hall busiticss

(j) casino tasiness, jackpot machines

(k) betting on horse and motorbike race

(I) golf course membership, playing fees

3.3. Taxes on Natural Resources

(a) ptoduction royalties

Cakculated on selling price at exploitation site duty-fiec lugggase

- goods in transit

- goods temporarily imported for ro-export.

- goods temporarily exported for re-impor

- goods imported for duty-free sale

Exemptions from or reduotion of execises granted in following cases:

(a) difficulties for enterprises producing excisable goods due to disaster, war, sudden calamity

(b) small-seale loss-making beer production (unti1 May 20, 2903)

(c) donictic automobile manufacturers are entitled to reduction from $50 \%$

to $100 \%$ until May 20, 2003, after which the period can be extended

by 1.5 years if they continuc to suffer lossen

(d) reduction of 30\% for golf conre businesses unti] May 20, 2001

Joint veniures partially exempted
$29 \%$ for cigarettes without filters

$45 \%$ for eigarettes with filters (mainly domestic materials) 65\% for cigarettes with filtems (mingly imported materials)

and cigars

$\$ 0 \%$ draft beer

$65 \%$ can ber

$75 \%$ bottle ber

$15 \%$ medicinal wine

$20 \%$ if below $20 \%$ alcohol (alcohol from fruit)

$25 \%$ if between 20 add $30 \%$ aloahol

$55 \%$ if between 30 and $40 \%$ alcoho

$70 \%$ if above $40 \%$ aleohol

$30 \%$ if $16-24$ seats

$60 \%$ if 6-15 stals

$100 \%$ if under $\$$ seat

$15 \%$

$20 \%$

$30 \%$

$20 \%$

$25 \%$

$20 \%$

2-5\% metallic minerals

I-4\% not-metallic minersls

2-3\% Bold

$4 \%$ rate soil

3-8\% gemstomes

$1-3 \%$ cosi

$6-25 \%$ oil

$0-10 \%$ gas

3-40\% natural forest products

2-10\% natural marine products

0-4\% natumal water

$0-20 \%$ other hatural resources 
(b) rent of land, waler, and sea surfaces

Payable by foreign-invealed enterpriges and contracting parties.

3.4. Withholding $T$ ax on Pyyuents in Respect of Intellectual Property

Royalties, license fees, matragement fees

Individuals bearing perbonal income tax

Companies bearing enterprise incorne tax

- Contractor bearing the rate of $10 \%$

\subsection{Import and Export Duties}

lmpart duty is bused on the contracted CF and d. a.f. price, exoept for 20 goods for which a mitimum import price has been delennined.

Import tasable value is determised as contract value matched with docurnents related to burying and sedling (CIF price); if import by road way,as buying price under Vietnam border conditions (DAF); except soms goods under the control of the State, taxable prict shall be applied aceording to minitnum price as regulated (currently incl. 15 groups of commodities)

- The regulation on import duty upon petrol, lubricant has many purposes: stabilizing price, encouraging investment to the domestic production of petrol/tubricant.

- Export taxable price is detertained as selling price at export gate (FOB)

-Minimum taxable value is tetermined by based on purchasing price in international markets on foreign trade contract of iasport goods of the same kind, roferencing domestic price of inport goods and onmments of Ministry of Trade and Custorus Department.

- Commodities subject to current export duty includes:

- Crude oil

- Wood and woody products

- cashewnut

- aloe-woo

- minerals

- precious and semi-precious gemstones

- particle of precious and semi-precious

- genstones

- metallic wastage

- uncotmpleted rtetals

tonprocessed leather, skin

- botanical materizh (rattan..)

- goods in transit

\section{Exempted imports are:}

- goods for national defense and security industries

- goods and services imponted for odication or scientific research

- goods toraporarily imported far subsequent export and thise

temporarily experted for fairs and exhibition

Additional tax-exempted imports-exponts are:

- imports, exports of moving assets

- sample of imports exports

- imports, exports of duty-free Inggage of passengers

- importis, exports of forcign organizations and individual

enjoying immmities and privileges in Vietnam under the regulation

of the Law of Vietnam, and in consistense with intemational convention

signed or attonded by Vietram

- imports for selling duty-fice

- imports, experts of foreign-funded enterprises and co-operating toreign parties undes the Law of Foreign Investment:

- imported equipment, machines to be fixed assets of enterprises

- special vehicles in imported tecinological chain to build up fixed assets and pick-up means (autonobiles with more than 24 seats)

- components, spare parts, appurtenances, appliances, moulds accompanied

the above-mentioned machines, equipment, vehicles

- imported materials used for projects of BOT, BTO, ET

- plant geeds, animals, special agrieultare chemicals permitted to imprort

to conduet agriculture, forestry and fishery projects

- other commodities, materials for investment encouragement special

projects under the regolations of the Government

- commodities sold by domestic organizations and individual

(including foreigr-funded enterprises) to processing enterprises

obtaining export duty exemption

- enterprises selling their-own products to other enterprises for producin

exports shall obtain import exemption for the materials related to

the abpve-mentioned products

- hunsanitarian aid; gifls as separately stipulated

Imports of equipment, machinery, and means of transportation are exempted

far the following investment projeets:

- afforestation and forest planting

- infrastructure and public trangpost
Depending on the particular conditiong in the locality US50.02/squarc meter - US\$12/square meter

(preferential or nocmal tates) : $0 \%, 1 \%, 3 \%, 5 \%$ $10 \%, 15 \%, 20 \%, 30 \%, 40 \%, 50 \%, 60 \%$; max $100 \%$

Ordinary rate, applied to non-MFN imports, $\max 70 \%$ higher than preferential rate

Preferential rate applied to MFN (and most) imparts

Enpecially prefesential rate applied to imports-frotin countries

with which Vietnam signed agreements to apply swch rate (mainly

for the Asean Free Trade Area).

Diseretionary additional tax is imposed in case of - import dumping

- imports subsidized by exporting country, thus hindering domestic production

- imports from countries imposing strict import tex policy on Vietnamese exports

Export duty rates:

- crude oil 4\%

- wood and woody products: $5 \%, 15 \%, 20 \%$

cashewnut: $4 \%$

- alocewood: $20 \%$

-minerals: $1 \%, 2 \%, 5 \%, 10 \%, 20 \%$

genstones: $1 \%, 3 \%, 5 \%$

-metallic wastage: $35 \%, 40 \%, 45 \%$ - semi-product metals: $2 \%, 5 \%, 10 \%$

- umprocessed leather: $10 \%$

- botanic materials (rattan ...): $3 \%$, $10 \%$ 


Nature of the tax
Import duty on gatoline aims to eliminate
between intemational and regulated dome
Export duty levied on f.o.b. value of:
- crude oil
- rubbet raw materials (e.g rubber milk)
- wood
- minarals (raw materials ard werap metal)

\subsection{Licene Tax}

Arunal licenate for all enterprises

\subsection{Slaughter Tax}

Based on rariket walue of slanghtered buffaloes,

eows, and pigs

\section{Praperty Taxes}

4.1. Agricultural Land Uве Tax

1. Based on area and category of agricultural land,

its tax rate is cakculated in paddy.

2. Category of land is determined based on following

- quality

- Location

- region (field, midd It, mountain)

- weather

- irrigation stuply and drainage situation

3. Category of land will be valid in ten years

4. Tax calculated in paddy but collected in

dong currency

Tax deductions and exemptions

Rates

- education, health care, sulture

- export production and trading

- Mgricultural and fishories development

- science, business management, technology transfer

- production teform ar relocation

- developing preferential professions

Inports for invesiment projects in regions with difficult or particulatly difficult

economic and sosial conditions aro exempted

Enterprises in "export processing zones" do not pay import and export

dutits on equipment, rww materials, and commodities imported into the zone

and for goods and products exported from the zone or sold to another EPZ

Domextic and foreign enterprises as well as partios to BCCs are exempted fiom impont durties on:

(a) imported equipment and machinery to form fixed agsets

(b) transport vehicks (cars with $24+$ teats, water transport vehicies)

to form fixed assels or transport workers

(c) raw and intermediate materials (incl. agricultural products)

(d) other goods for projects on list of encouraged projects

Minimurt annual tax VND25,000

if monthly incotide $<$ VND 150,000 .

Maximum annuel ix VID850,000

if rmogthly income > VND1, 250,000

$5 \%$

Exempt are:

1. Bare hills and land used for agricultural production

2 Reclaimed land used for

- annual crops: 5 years; 7 years of exemption will be applied for

marsh, sez-encraaching areas

- perennial crops, duritig capital construction plus 3 years since

crops start yieiding outputs. 6 years for morsh and seiteneroaching areas.

3 Targot groups of social policies:

- for farmer howeholds in mountainous, border and island areas and of minorities

- invalid or elderly famers houschold wiftout any support

- martyr families that are being subsidized by the State

- hotrseholds of war invalids of the level $1 / 4$ and $2 / 4$; sick soldiexs of

the level 1/3 and 2/3.

4. Tax reduction will be considered for other types of household of difficulties.

5. Tax exemption and reduction due to salamicies: Tax on agricuthural land use

would be exempted or reduced an crop basis as follows:

- darnage from 10\%. 20\%: tax reduetion of according late of damage

- dama ge from $20 \%$ - $<0 \%$; reduction of $60 \%$

(CInternational Monetary Fund. Not for Redistribution

1 Annual erops

\begin{tabular}{cc} 
Categowy of land & Tax (kg paddy/hectare) \\
\hline 1 & 550 \\
2 & 460 \\
3 & 370 \\
4 & 280 \\
5 & 180 \\
6 & 50
\end{tabular}

2 Peremial and leng-term:

\begin{tabular}{cc} 
Categoryef land & Tax (kg paddy/hectare) \\
\cline { 2 - 2 } 1 & 650 \\
2 & 550 \\
3 & 400 \\
4 & 200 \\
5 & 80
\end{tabular}

3 As for wood tree and perennial trees that 
v. Vletnam: Summary of the Tax System as of January 1, 2000

- damaje frora $30 \%-40 \%$ reduction of $80 \%$

- damage from $40 \%$ and more: tax exemplect $(100 \%)$

\subsection{Land and Honsing Tax}

1. Based on : area, eategory of land and the tax rate of agricultural land use

2. Tux is caloufated by paddy but collected

in dong surrency

4.3. Land Use Right Transfer Tax

\subsection{Registration Fees}

Assets of organizations and individuals in the groups

that have to register the ownership and user-rights,

subject to registration fees includes:

- Housing land

- Boats, autamobiles, motercyeles

- Hunting guns, sport guns.

\section{Local Fees}

Fees and tolls not shared with higher levels of government
Exempt are:

- Lathd uned for building of adminintrative offjes ar public works

- Residential land in mountainous, highland, border, island and remole areas

- Residential land of war invalids of level 1/4 and 2/4

- Martyr households that are subsidized by the State

Tex reduction or exemption will be applied for honseholds of difficulties

tue to calamities, aceidenta. If damage value is $20 \%-50 \%$ of the total value

on the land area, then land tax reduction of $50 \%$ will be considered.

If the loss yalue is $100 \%$, tax exemption will be considered.

\section{Exempt are}

- Goversument allocates land for orgenizations, househalds and individuals by Law

- transfers of househalds on individuals moving to new economic zones,

mountainous areas, islands under the decision of competent autbocities

- womer who ane "Vietnam Heroic Mother"

- tratuffets in communes of mountaincus areas, islands as regulated by

the Government

- swap between agricultural, forestry, fisheries and salt production land

$50 \%$ reduction for

- war invalid of level $1 / 4$ and $2 / 4$; sick soldien of level $1 / 3$ and $2 / 3$

- mantyr households that are anbsidized by the State

- bandicrpped, pre-rnature and elderly people with no support are harvested one time only, tax rate is $4 \%$ of the output vahue.

- The minimun rate is the tverage level of a gricultural land uss tax recorded in the commune.

- Highest rate will be $\$ 2$ times of agrieultural land use tax

- Land in urbar areas; from 3 - 32 times.

- Land in outskirts, along roads, near commercial centers, tourisim sreas, new industrial areas, the land tax rate will be from $1.5-2.5$ times.

- Land in rural areas: land tax nte will be the average level of the agriealtaral land use tax recorted in the commune.

- Land used for agricultural, forestry, fisheries and salt production: $2 \%$

- Land uged for residence, wark construction and others: 4\%

Sources: Vietnamese authorities, Deloitte Tonche Tohmatsu International, Coopers \& Lybeand, Price Watethouse 